\title{
Effectiveness of mifamurtide in addition to standard chemotherapy for high-grade osteosarcoma: a systematic review
}

\author{
A thesis submitted by \\ Rincy Jimmy \\ in partial fulfilment of the requirements for the degree of \\ Master of Clinical Science (MClinSc)
}

The Joanna Briggs Institute

Faculty of Health and Medical Sciences

The University of Adelaide

Australia 


\section{Table of Contents}

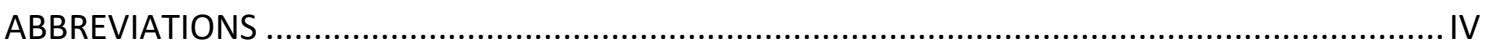

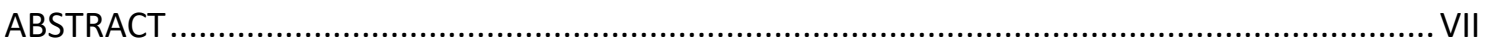

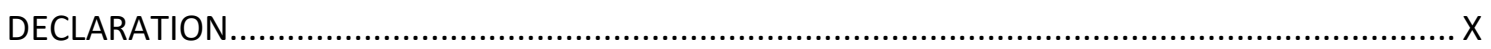

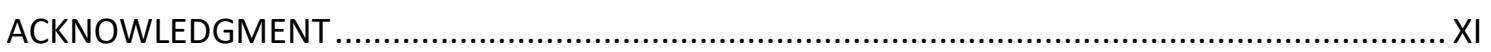

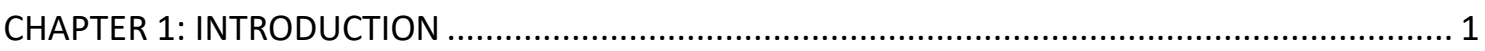

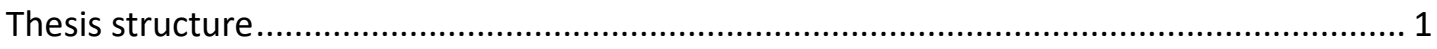

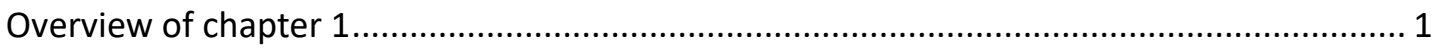

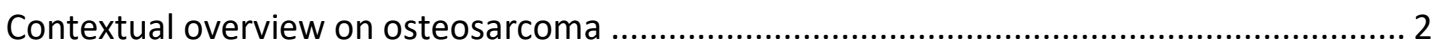

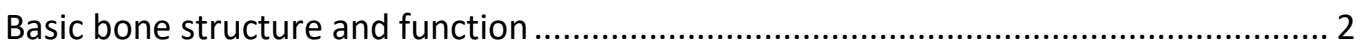

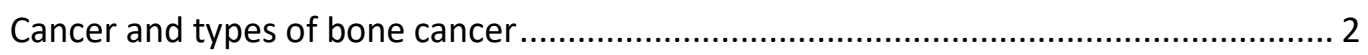

Classification and histomorphology of osteosarcoma ................................................... 3

Osteosarcoma staging system and statistics ............................................................ 4

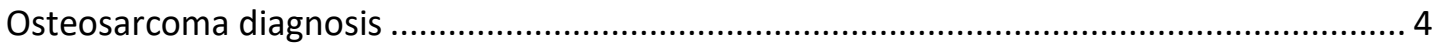

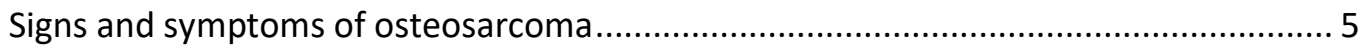

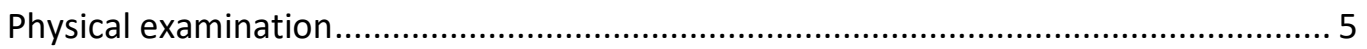

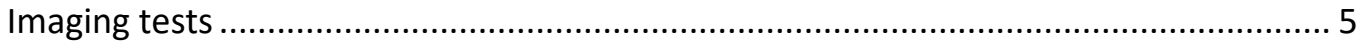

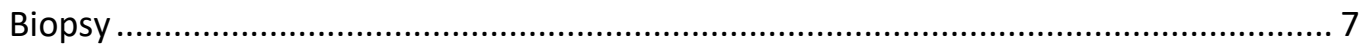

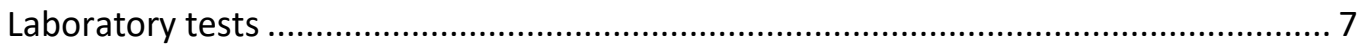

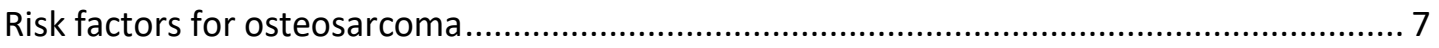

Age

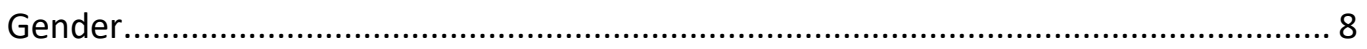

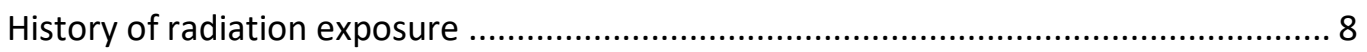

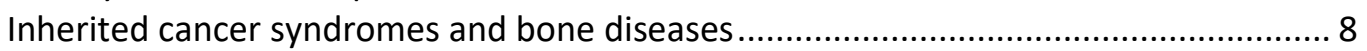

Current treatment regimen for high-grade osteosarcoma................................................ 9

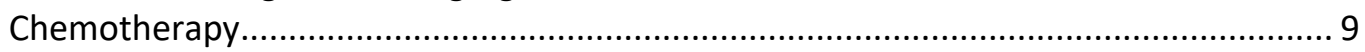

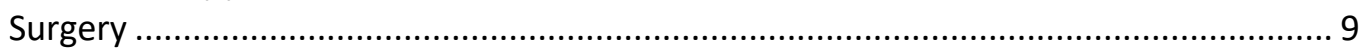

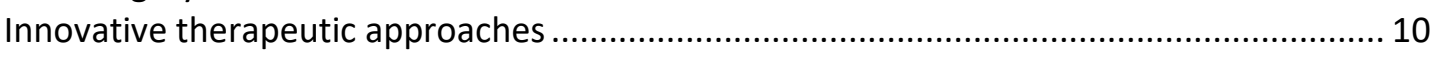

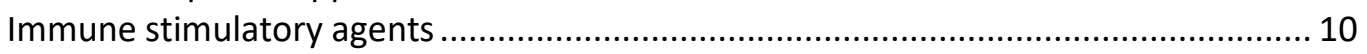

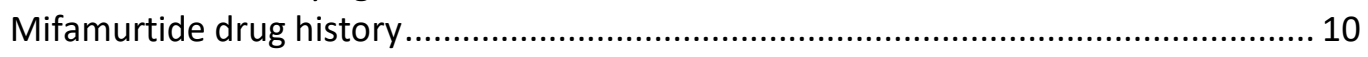

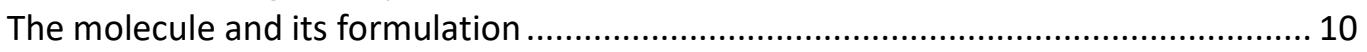

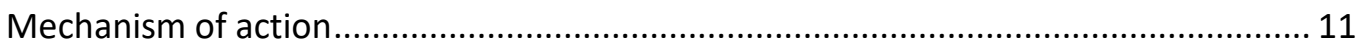

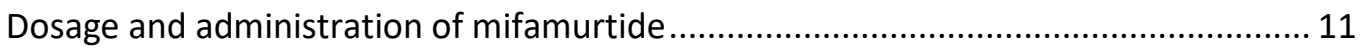

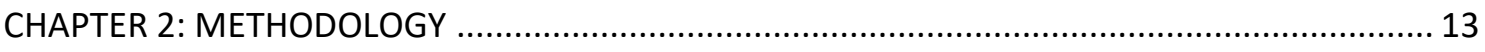

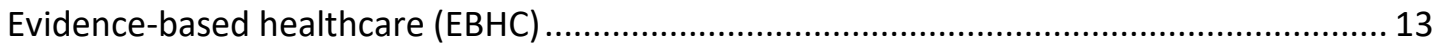

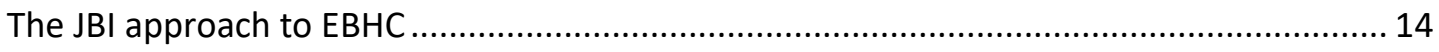

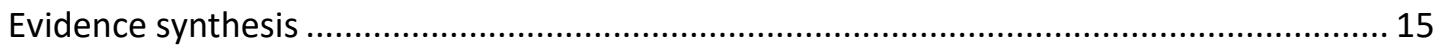

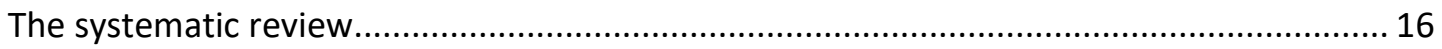

Levels of evidence and the grading of recommendations .................................................. 17

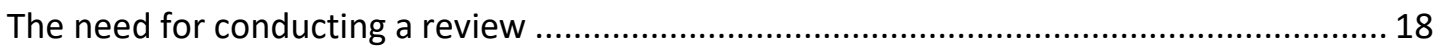

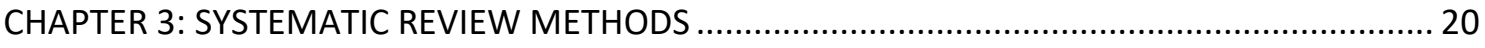

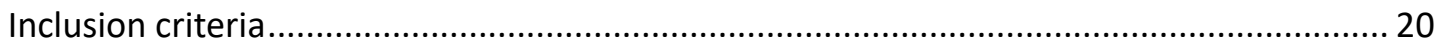

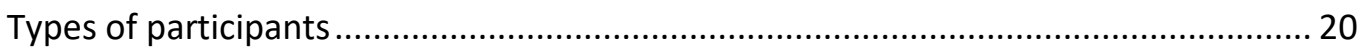

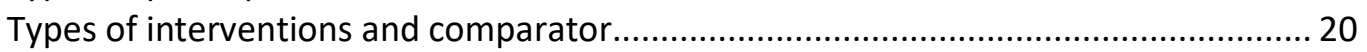




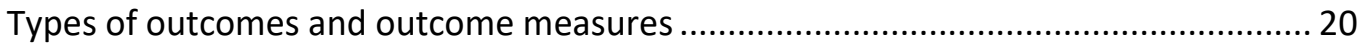

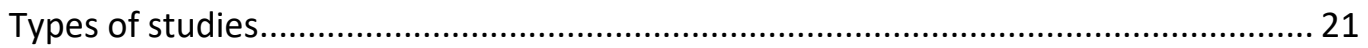

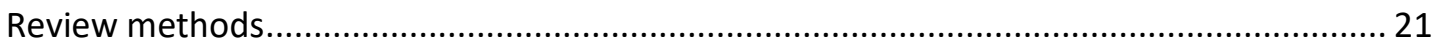

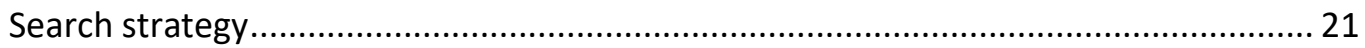

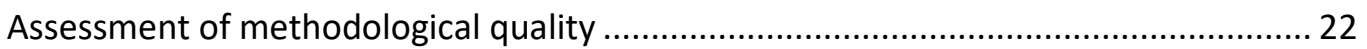

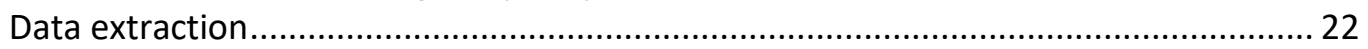

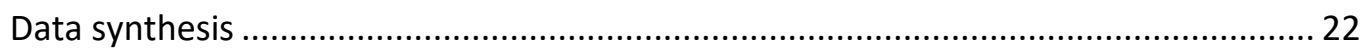

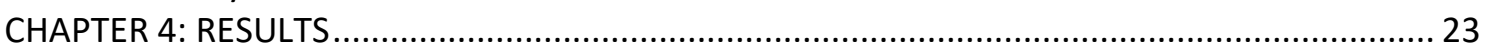

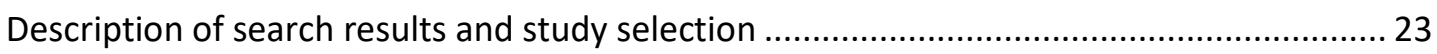

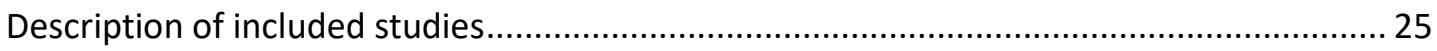

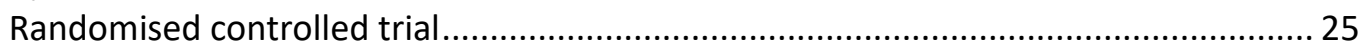

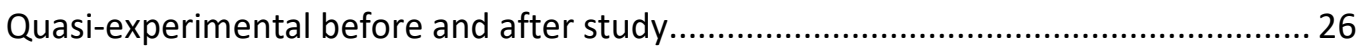

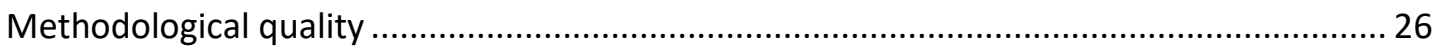

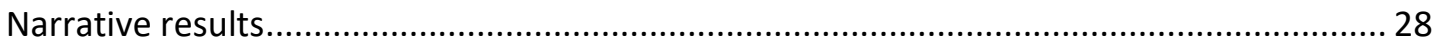

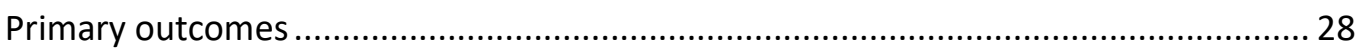

Secondary outcomes

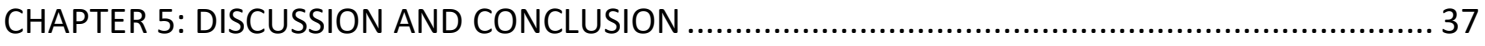

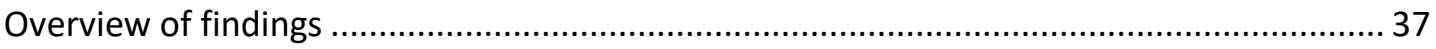

Effectiveness of mifamurtide in addition to standard chemotherapy on EFS for high-

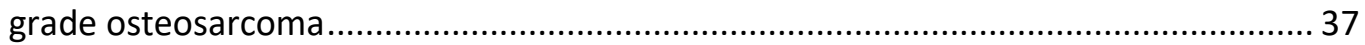

Effectiveness of mifamurtide in addition to standard chemotherapy on overall

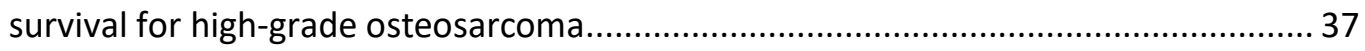

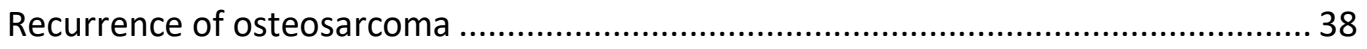

Effectiveness of mifamurtide in addition to standard chemotherapy on

mifamurtide-related adverse events and HRQoL for high-grade osteosarcoma............ 38

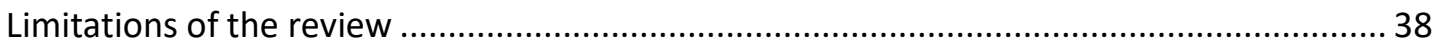

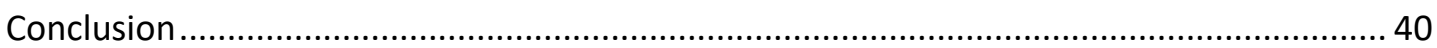

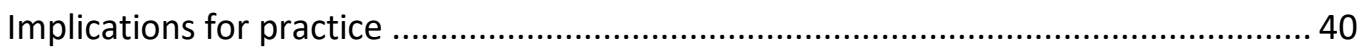

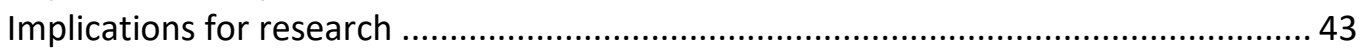

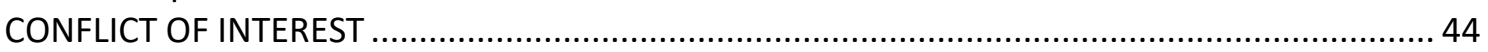

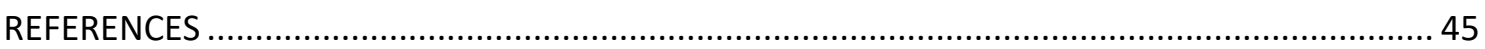

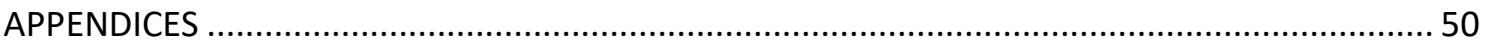

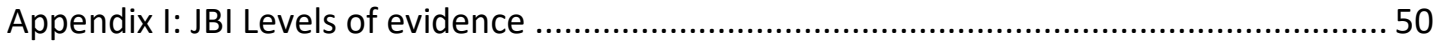

Appendix II: New JBI Grades of Recommendation .......................................................... 51

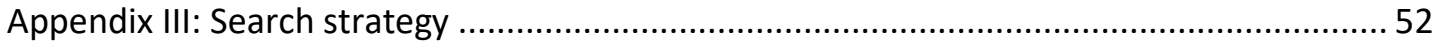

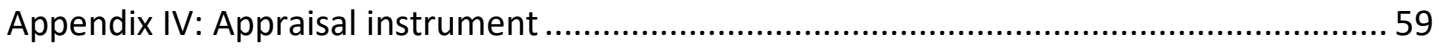

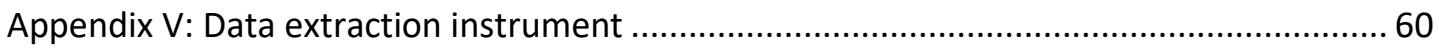

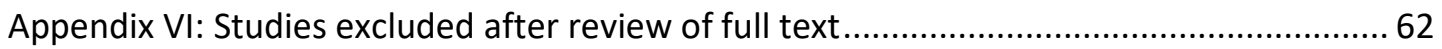

Appendix VII: Characteristics of included studies ............................................................ 72 


\section{List of tables}

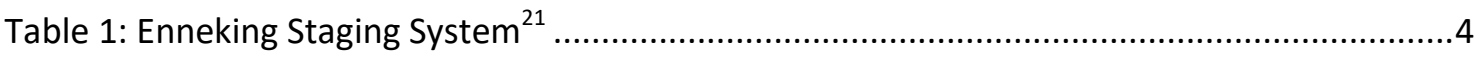

Table 2: Application of GRADE quality of evidence in the GRADE approach..........................18

Table 3: Results of critical appraisal of included randomised controlled trial/pseudo-

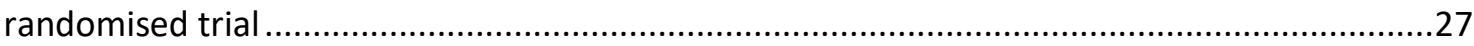

Table 4: Five-year EFS in metastatic osteosarcoma patients according to treatment regimen

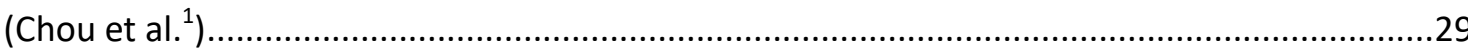

Table 5: Four-year and six-year EFS in non-metastatic osteosarcoma patients according to treatment regimen (Meyers et al. $^{2}$ ) ...........................................................................30

Table 6: PFS in pulmonary metastatic osteosarcoma patients according to treatment regimen

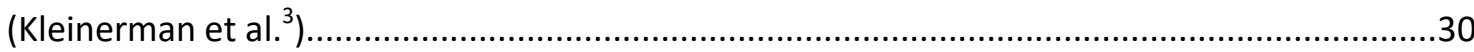

Table 7: Five-year overall survival in metastatic osteosarcoma patients according to treatment

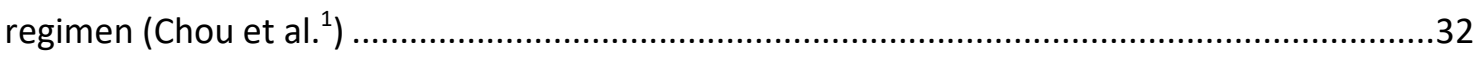

Table 8: Four-year and six-year overall survival in non-metastatic osteosarcoma patients according to treatment regimen (Meyers et al. ${ }^{2}$ )

Table 9: Survival after relapse in pulmonary metastatic and/or relapsed osteosarcoma patients according to treatment regimen (Kleinerman et al. ${ }^{3}$ ) .......................................................33

Table 10: Mifamurtide-related adverse events in metastatic osteosarcoma patients according

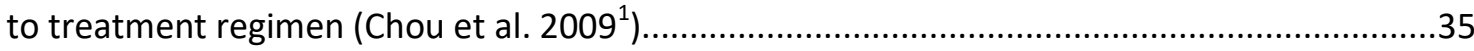

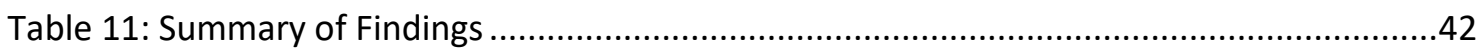

\section{List of figures}

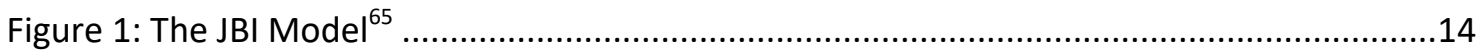

Figure 2: Flow chart for identification of studies for inclusion and exclusion .........................24 


\section{ABBREVIATIONS}

ARTG - Australian Register of Therapeutic Goods

BLM - Bloom syndrome

CCG - Children's Cancer Group

COG - Children's Oncology Group

$\mathrm{Cl}$ - Confidence Interval

CT - Computed tomography

DNA - Deoxyribonucleic acid

EBHC - Evidence-based healthcare

EFS - Event-free survival

EMA - European Medicines Agency

FAME - Feasibility, Appropriateness, Meaningfulness and Effectiveness

FDA - Food and Drug Administration

GRADE - Grading of Recommendations, Assessment, Development and Evaluation

HDMTX - High-dose methotrexate

HER2 - Human epidermal growth receptor-2

HRQoL - Health-related quality of life

HR - Hazard ratio

IGF1R - Insulin-like growth factor receptor 1

IL-1 - Interleukin 1

IL-6 - Interleukin 6

IL-8 - Interleukin 8

IRAEs - Infusion-related adverse events

JBI - Joanna Briggs Institute

JBI-SUMARI - Joanna Briggs Institute System for the Unified Management, Assessment and Review of Information 
JBI-MAStARI - Joanna Briggs Institute Meta Analysis of Statistics Assessment and Review Instrument

JBISRIR - Joanna Briggs Institute Database of Systematic Reviews and Implementation Reports

LDH - Lactate dehydrogenase

L-MTP-PE - Liposomal muramyl tripeptide phosphatidylethanolamine

$\mathrm{mCi}-$ Millicurie

MDACC - MD Anderson Cancer Center

MRI - Magnetic resonance imaging

MeSH - Medical Subject Headings

MTP - Muramyl tripeptide

MTP-PE - Muramyl tripeptide phosphatidylethanolamine

MDP - Muramyl dipeptide

MTD - Maximum tolerated dose

NOD2 - Nucleotide-binding oligomerisation domain-containing protein 2

$\mathrm{NaCL}$ - Sodium Chloride

NCCN - National Comprehensive Cancer Network

$\mathrm{NCl}$ - National Cancer Institute

NF - Nuclear factor

NPP - Named Patient Program

OR - Odd Ratio

PET - Positron emission tomography

PICO - Population, Intervention, Comparator and Outcomes

PFS - Progression-free survival

POG - Pediatric Oncology Group

$\mathrm{pRb}$ - Retinoblastoma protein

RecQ - Structurally-related DNA helicase

RECQL - gene encoding one member of a protein family called RecQ helicases 
RNA - Ribonucleic acid

RCTs - Randomised controlled trials

$\mathrm{RR}$ - Relative risk

SPSS/PC+ - Statistical Package for the Social Sciences/PC+

TP3 - Therapeutic antibody administration

TGA - Therapeutic Goods Administration

TNF- $a$ - Tumour necrosis factor-alpha

Tc99m MDP - technetium 99m methylene diphosphonate

WHO - World Health Organisation

WRN - Werner syndrome 


\section{ABSTRACT}

\section{Background}

Osteosarcoma mostly occurs during the period of rapid bone growth in children and adolescents as high-grade osteosarcomas. Current treatment recommended for high-grade non-metastatic and metastatic and/or relapsed osteosarcoma involves neoadjuvant multiagent conventional chemotherapy, followed by surgical resection of macroscopically detected tumour and postoperative adjuvant chemotherapy. However, residual micrometastatic deposits that develop following surgery have shown resistance to postoperative/adjuvant chemotherapy. Therefore, there is a critical need for more effective and innovative therapeutic approaches such as immune stimulatory agents. The most extensively studied immune stimulatory agent in the treatment of osteosarcoma is mifamurtide. The aim of this systematic review was to identify and synthesise the evidence on the effectiveness of mifamurtide in addition to standard chemotherapy on survival outcomes.

\section{Objectives}

To present the best available evidence related to the treatment of high-grade non-metastatic and metastatic osteosarcoma with mifamurtide in addition to standard chemotherapy.

\section{Inclusion criteria}

Types of participants

All populations of patients, regardless of age, gender or ethnicity with high-grade, resectable, non-metastatic and metastatic osteosarcoma based on histological diagnosis.

\section{Types of interventions and comparators}

This review focused on intravenous infusion of either of the pharmaceutical formulations of mifamurtide (MTP-PE or L-MTP-PE) in addition to standard chemotherapy, and the comparator was chemotherapy alone.

\section{Types of studies}

This review considered any experimental study design including randomised controlled trials, non-randomised trials and quasi-experimental studies.

\section{Types of outcomes}

The primary outcomes of interest were event-free survival, overall survival and recurrence of osteosarcoma. Secondary outcomes that were considered included health-related quality of life and any mifamurtide-related adverse events.

\section{Search strategy}

A search for published and unpublished literature in the English language was undertaken (seven published literature databases, four unpublished literature databases, and three government agency and organisational websites). Studies published between 1990 to June 2016 were considered. A three-step strategy was developed using MeSH (Medical Subject Headings) 
terminology and keywords to ensure that all relevant studies related to this review were included.

\section{Methodological quality}

The methodological quality of included studies was assessed by two reviewers, who appraised each study independently, using a standardised Joanna Briggs Institute (JBI) critical appraisal tool.

\section{Data extraction}

Data was extracted from the studies that were identified as meeting the criteria for methodological quality using the standard JBI data extraction tool.

\section{Data synthesis}

Due to the heterogeneity of populations and interventions and available studies, meta-analyses were not possible and results are presented in narrative form.

\section{Results}

Three papers outlining two studies involving 802 patients evaluated the effectiveness of mifamurtide in addition to chemotherapy. Results indicated no significant difference in event-free survival between the addition of mifamurtide to standard chemotherapy regimens and chemotherapy alone, both in non-metastatic and metastatic osteosarcoma patients. There was a significant difference in progression-free survival favouring the addition of mifamurtide in pulmonary metastatic and/or relapsed osteosarcoma. There was no significant difference in overall survival between the addition of mifamurtide and chemotherapy alone in metastatic osteosarcoma; however there was a significant difference favouring the addition of mifamurtide in non-metastatic osteosarcoma patients. The addition of mifamurtide resulted in a significant difference in survival after relapse in pulmonary metastatic and/or relapsed osteosarcoma patients. Both studies reported on mifamurtide-related adverse events - the first was reported as toxicity which included haematological, hepatic, renal, gastrointestinal disorders, cardiac rhythm, nervous system disorders, ear disorders and others (infection, fever and performance status) in metastatic osteosarcoma patients. Results were similar across all combined treatment regimens. Although no statistical analysis was undertaken, the figures suggest there were no significant differences between the treatment regimens. In the other study, mifamurtide-related adverse events were reported as clinical toxic effects of mifamurtide in relapsed osteosarcoma, which included chills, fever and headache for the initial dose of mifamurtide, while for the subsequent doses of mifamurtide all patients reported toxicity as delayed fatigue.

\section{Conclusions}

The available evidence on the effectiveness of mifamurtide in addition to a standard chemotherapy regimen for the treatment of high-grade osteosarcoma is limited and therefore no definitive conclusions can be made.

\section{Implication for practice}

There is currently limited evidence to recommend or refute the addition of mifamurtide to the standard chemotherapy regimen for the treatment of high-grade osteosarcoma. 
Implication for research

Additional high quality studies such as randomised controlled trials or quasi-experimental studies involving a larger sample size are required. Consistency in outcome measures is critical to facilitate comparison.

Cost-effectiveness studies of mifamurtide are required to inform choice from a societal perspective.

\section{Keywords}

Osteosarcoma, osteogenic sarcoma, mifamurtide, 'muramyl tripeptide phosphatidylethanolamine', 'muramyl tripeptide'. 


\section{DECLARATION}

I certify that this work contains no material which has been accepted for the award of any other degree or diploma in my name, in any university or other tertiary institution and, to the best of my knowledge and belief, contains no material previously published or written by another person, except where due reference has been made in the text. In addition, I certify that no part of this work will, in the future, be used in a submission in my name, for any other degree or diploma in any university or other tertiary institution without the prior approval of the University of Adelaide and where applicable, any partner institution responsible for the joint award of this degree.

I give consent to this copy of my thesis, when deposited in the University Library, being made available for loan and photocopying, subject to the provisions of the Copyright Act 1968.

I also give permission for the digital version of my thesis to be made available on the web, via the University's digital research repository, the Library Search and also through web search engines, unless permission has been granted by the University to restrict access for a period of time.

Rincy Jimmy:

Date: 


\section{ACKNOWLEDGMENT}

I acknowledge and thank the Joanna Briggs Institute for providing me with extended support to undertake the critical learning course work to enable me to commence the systematic review process.

A big thank you to my academic supervisors, both past and present, from the Joanna Briggs Institute: Dr Sarahlouise White, Dr Karolina Lisy and Dr Cindy Stern. I have learned so much from your expertise and many thanks for the considerate support and encouragement throughout the course.

I offer my appreciation to Dr. Catalin Tufanaru for statistical advice.

A special thank you is reserved for my husband and children who have encouraged me throughout, with pride and enthusiasm, to achieve this body of work, and who have allowed me the indulgence of time to complete it. Thank you to my parents, who have been motivational, and also friends, who have assisted my family in every way possible to enable me to indulge in this process.

Most importantly, being a member of Cancer Voices SA, and from a consumer perspective, I dedicate this work to Joby Joseph, my beloved brother, who lost the battle against osteosarcoma. A contributor and keeper of knowledge, not much was known to him as a consumer, but he remains my inspiration for continual learning. 


\section{CHAPTER 1: INTRODUCTION}

The focus of this thesis is the presentation of a systematic review following the Joanna Briggs Institute (JBI) methodology for reviewing evidence on the effectiveness of an intervention. The review question sought to identify and synthesise the best available evidence on the effectiveness of mifamurtide in addition to standard chemotherapy on event-free survival, overall survival, recurrence of osteosarcoma, mifamurtide-related adverse events and health-related quality of life for patients with high-grade, resectable, non-metastatic and metastatic osteosarcoma.

\section{Thesis structure}

This thesis is organised into the following five chapters:

Chapter 1: Introduction: This first chapter provides a background to the topic of interest osteosarcoma. Risk factors, diagnosis and current standard treatment regimens are described. An overview of mifamurtide as a possible treatment option is provided.

Chapter 2: Methodology: The second chapter addresses the methodological principles upon which the systematic review of international literature is based. This includes a description of the development and origins of evidence-based healthcare, evidence synthesis and the systematic review.

Chapter 3: Systematic review methods: This chapter describes the methodological process undertaken in the systematic review underpinning this thesis. This chapter describes the review objective/questions, types of studies, participants, interventions and comparators, and the outcomes and outcome measures of interest. The search strategy is detailed, alongside the appraisal process for methodological quality, the process of data extraction and the method of data synthesis.

Chapter 4: Results: The fourth chapter describes the search results and the methodological quality and characteristics of included studies. The findings of the review are presented.

Chapter 5: Discussion, conclusions and recommendations for practice and research: The fifth chapter discusses the main findings generated from the systematic review, the limitations of the review, the conclusions, and the implications for practice and research.

\section{Overview of chapter 1}

This first chapter of the thesis is broken down into the following sections: the organisation of this thesis (section 1.1); outline of chapter 1 (section 1.2); overview on osteosarcoma with reference to the basic structure and function of bones, cancer and different types of bone cancer, and 
classification and the staging systems of osteosarcoma, (section 1.3); diagnosis of osteosarcoma (section 1.4); risk factors of osteosarcoma (section 1.5); current treatment regimen for high-grade osteosarcoma (section 1.6); and an outline of innovative therapeutic approaches including immune stimulatory agents, mifamurtide drug history, formulation and mechanism of action, and finally dosage and administration of mifamurtide (section 1.7).

\section{Contextual overview on osteosarcoma}

This section provides a brief synopsis of the general structure and function of bones in order to understand the complexity that occurs in osteosarcoma.

\section{Basic bone structure and function}

Bones are the structural support of the skeletal system which have a number of specific functions in the human body. ${ }^{4}$ There are different types of bones such as long bones, short bones, flat bones and irregular bones, however, this thesis discusses only those relevant to osteosarcoma. Flat bones provide mechanical protection to vital organs such as skull bones which protect the brain, and breast bone and ribcage which protect the heart and lungs. ${ }^{4}$ Long bones are the bones of the arms and legs which are connected to muscles that help in movement of limbs.

Bones are internally made up of active tissues and bone marrow. ${ }^{4}$ New blood cells are produced in the bone marrow, while minerals such as calcium and phosphorus are stored in bone tissue. ${ }^{4}$ Gradually these bones become more rigid, complex and harden with age. The active tissues of bone are mainly made up of two different types of bone cells: osteoblasts and osteoclasts. ${ }^{5}$ Both osteoblasts and osteoclasts play a major role to keep the bone structure intact. ${ }^{5}$ Osteoblasts are the mesenchymal cells, also referred to as the building blocks of bone, located within bone marrow stroma which help to deposit the bone matrix, a process known as mineralisation of bone. ${ }^{5,6}$ Osteoclasts break down any excess bone matrix, a process known as reabsorption of bone, thereby maintaining the structural shape of bone.5,7 In addition to depositing and breaking down minerals, they also maintain blood calcium levels in the body. Uncontrolled and immature osteoblasts within bone tissue can affect the up-regulation of osteoclast differentiation and bone reabsorption function that can lead to the development of bone cancer. ${ }^{5}$

\section{Cancer and types of bone cancer}

Cancer is a disease of cells. Within a healthy body, cell growth is controlled, however, when such controlled growth is lost, cancerous cells arise and have the potential to spread within the body. Primary bone cancer originates in cells that grow bone tissue. Types of primary bone cancers include osteosarcoma, Ewing's sarcoma, malignant fibrous histiocytoma and chondrosarcoma.8,9 Osteosarcoma is the most common type of primary bone cancer that can occur in children and young adults. ${ }^{10,11}$ Ewing's sarcomas are the second most common primary bone cancer, seen in children, ${ }^{9,12}$ whereas the other two types of primary bone cancer mainly occur in adults. Primary bone cancers are a rare type of cancer. ${ }^{9}$ Cancer that has spread from another part of the body to the bone is called secondary bone cancer. Secondary bone cancer is treated differently to primary bone cancer. 


\section{Classification and histomorphology of osteosarcoma}

Osteosarcoma, also called osteogenic sarcoma, is an osseous malignant tumour of osteoid tissue that starts in the bones. ${ }^{11}$ There are two main types of osteosarcoma, primary osteosarcoma and secondary ostoeosarocma. ${ }^{13}$ Primary osteosarcomas are classified based on their origin intramedular/central or juxtacortical/surface osteosarcoma. Based on their histological appearance, intramedular osteosarcomas have a predominant matrix or stoma cell types which are microscopically different from normal bone tissue. ${ }^{12}$ This tissue is derived from mesenchymal cells which exhibit osteoblastic differentiation and produce spindle-shaped malignant osteoid. 14,15 The most commonly noted feature is the new developing immature bone (also called osteoid), involving predominantly metaphysis of the long bones. ${ }^{11,16,17}$ It often develops at the region of bone growth, ${ }^{18}$ particularly, the terminal ends of the long bones of the arms and legs, such as near the knee. Osteosarcomas may also develop in the distal femur, the proximal tibia, the proximal humerus or in any bone of the body. In older adults it commonly develops in the pelvis (hips), ${ }^{19}$ shoulder and jaw. In osteosarcoma, uncontrolled osteoblast activity leads to irregular osteoclast differentiation and function that may cause cancerous growth in the bone tissue. ${ }^{5}$ The general tendency of osteosarcoma is to metastasise with considerable diversity in histologic features.

Osteosarcoma is a rare type of cancer that differs greatly in its biology ${ }^{15}$ and behaviour; its origin is unknown. According to the Enneking Staging System and the World Health Organisation (WHO),9,19,20 osteosarcoma can be classified into high-grade, intermediate-grade or low-grade, based on histological characteristics. ${ }^{8}$

High-grade osteosarcomas: High-grade osteosarcomas are the fastest growing type of osteosarcoma. Most osteosarcomas that occur in children and adolescents are high grade. There are different histological subtypes of high-grade osteosarcoma, which include conventional, osteoblastic, chondroblastic, fibroblastic, mixed, small cell, epithelioid, giant cell-rich, telangiectatic, dedifferentiated parosteal and high-grade surface (juxtacortical high grade). ${ }^{9,21-23}$ Other high-grade osteosarcomas include pagetoid (a tumour that develops in someone with Paget disease of the bone ${ }^{11}$ ), extra-skeletal (a tumour that starts in a part of the body other than a bone), and postradiation (a tumour that starts in a bone that had once received radiation therapy).9,21-23 The three most common subtypes of high-grade osteosarcoma (osteoblastic, chondroblastic and fibroblastic) account for $80-90 \%$ of osteosarcoma diagnoses. ${ }^{9,20-26}$

Intermediate-grade osteosarcomas: There is only one type in this grade - periosteal, which is also known as juxtacortical. ${ }^{9}$ It is highly uncommon to develop this type of tumour which is very similar to low-grade osteosarcoma.

Low-grade osteosarcomas: Low-grade osteosarcomas are the slowest growing type of osteosarcoma. This type of tumour is uncommon but once developed, it is unresectable. There are two types, low-grade central and parosteal osteosarcoma. ${ }^{9}$

This thesis focuses on the most common of the three types of high-grade osteosarcoma. 


\section{Osteosarcoma staging system and statistics}

Often diagnosis of osteosarcoma involves radiography, imaging studies and laboratory tests, later confirmed by biopsy and tumour pathology. ${ }^{18,23}$ There are three basic steps involved in the staging of osteosarcoma. The first step is to determine the histological feature, which is known as the grade that identifies the biological aggressive nature of tumour. The second step is to ascertain how extensive the tumour is, whether or not it has advanced elsewhere outside the compartment of origin, through bone, into the surrounding soft tissue. The last step relates to whether the tumour has spread to another part of the body (i.e. has it metastasised). Metastatic osteosarcomas can be detected by imaging studies, and mainly spread to the lungs, lymph nodes, and another location within the bone or to other bones. Following the initial imaging and laboratory examination, a biopsy is conducted either by needle biopsy or through open methods, in order to make a conclusive diagnosis. Following the pathological evaluation, a histologic grade is assigned that determines the characteristic of the tumour. In musculoskeletal oncology, one of the most frequently used staging systems is the Enneking Staging System which takes into account the intra- or extra-compartmental location of the tumour. ${ }^{21}$ According to the Enneking Staging System (Table 1), low-grade tumours are stage-I, well differentiated, with less than $25 \%$ risk of metastasis, and subdivided as A and B, based on the involvement of the anatomical compartment. High-grade tumours are stage-II, poorly differentiated, have high cell to matrix ratio, and subdivided as A and B. Stage-IA and IIA are contained in well-defined compartments (referred to as intracompartmental) while stage-IB and IIB tumours extend beyond the compartment (extracompartmental). Stage-III consists of tumours with metastasis regardless of the size and grade. Based on this system, the most commonly seen stage of osteosarcoma is stage IIB.12,13 When diagnosed, $70-80 \%$ of patients present with localised disease (non-metastatic), while $20-30 \%$ of patients present with metastatic osteosarcoma. ${ }^{15,24} \mathrm{At}$ least $80 \%$ of recurrent osteosarcoma develops in the lungs and approximately $15 \%$ in distant bones. ${ }^{27}$

Table 1: Enneking Staging System ${ }^{21}$

\begin{tabular}{|l|l|l|}
\hline Stage & Grade & Site \\
\hline IA & Low & Intracompartmental (T1) \\
\hline IB & Low & Extracompartmental (T2) \\
\hline IIA & High & Intracompartmental (T1) \\
\hline IIB & High & Extracompartmental (T2) \\
\hline III & Any grade & Any T, metastasis \\
\hline
\end{tabular}

\section{Osteosarcoma diagnosis}

Most cases of osteosarcoma are identified by histopathological diagnosis. ${ }^{12}$ However, complete clinical evaluation of any suspected osteosarcoma primarily involves signs and symptoms, physical examination, and results of different imaging tests and laboratory tests which may indicate any developing or existing primary bone cancer. ${ }^{12}$ Osteosarcoma can be further confirmed with pathological examination by biopsy that examines the tissue or cell sample from the suspected area 
of bone. ${ }^{12}$ Accurate diagnosis of a primary bone cancer often depends on the location of the tumour and bone, chest $\mathrm{x}$-ray reports and biopsy. In cases of metastatic osteosarcoma, diagnosis also includes signs and symptoms, and biopsies that are done to diagnose the initial bone metastasis. ${ }^{12}$

\section{Signs and symptoms of osteosarcoma}

The clinical presentation of osteosarcoma begins with local pain 15,19 and is often mistaken for muscular soreness or "growing pains". 8 Typical signs and symptoms of osteosarcoma include pain typically present for several months before diagnosis, ${ }^{18}$ sometimes followed by localised palpable mass or swelling15,20 over the involving region of bone or a part of the body limiting joint movement. 19,23 Many patients present to a doctor with pain and abnormality of the bone following an injury to the affected area or when a pathological fracture occurs, particularly in patients with osteolytic tumours. ${ }^{19}$

\section{Physical examination}

Patients with signs and/or symptoms of a tumour in or around the region of bone are required to seek further medical attention to assess overall health and well-being. ${ }^{12} \mathrm{~A}$ physical examination generates information on general physical health and can indicate development of a soft tissue clump at the primary tumour site. ${ }^{12}$ In cases where the physician interprets that the patient might have osteosarcoma, more tests are subsequently performed, including imaging tests, biopsies and/or laboratory tests.

\section{Imaging tests}

Imaging tests may include X-rays, magnetic fields or radioactive substances to create an internal image of the body. Imaging tests are conducted to: (1) identify a suspected area for development of a tumour, (2) determine whether the tumour has advanced to another part of the body, (3) further investigate the spread of the tumour, (4) determine whether the treatment has been effective, and (5) check for any signs of recurrence. The common imaging tests undertaken in osteosarcoma diagnosis, which includes bone and chest X-ray, cross-sectional imaging techniques such as Tc99 bone scan ${ }^{13,19}$ (specifically in case of metastatic osteosarcoma), computed tomography (CT) scan or magnetic resonance imaging (MRI). ${ }^{18,23}$

Bone and chest $X$-ray: In the majority of cases, bone cancer is indicated on a plain radiography bone $X$-ray that can suggest a diagnosis of osteosarcoma. ${ }^{21}$ The bone at the site of the tumour may appear tattered rather than compact. Radiographic appearance of the affected area of bone may show a lytic, sclerotic or mixed lytic-sclerotic lesion ${ }^{12}$ with irregular margins, a sun burst appearance (radial ossification in the soft tissue), ${ }^{12,13}$ a Codman triangle ${ }^{13,19}$ (considered typical in osteosarcoma), and new bone formation in the region of soft tissue along with destruction of the cortex. ${ }^{13,18}$ The radiologist then determines whether a tumour is malignant based on its appearance on the bone X-ray, which is later confirmed by biopsy. Secondly, a plain radiography of the chest is performed, known as a chest X-ray, and is often done to check whether the primary bone cancer has spread to the lungs. 
Computed tomography (CT) scan: CT scans produce detailed three dimensional, cross-sectional imagery of the tumour that defines the exact location and its extent that assists at the time of surgical intervention. 12,18,23 Unlike conventional X-ray, a CT scan takes different images as it revolves around the thoracic region and the computer combines these images into a single image (also known as a spiral CT scan of the thorax). This further assists in clinical detection of pulmonary metastatic osteosarcoma at the time of diagnosis. Additionally, it is also helpful in staging osteosarcoma and identifying the spread of primary bone cancer. ${ }^{13}$ Furthermore CT scans are used to guide the needle biopsy into a suspected location such as the lungs to check the metastatic spread at the site. ${ }^{8}$

Magnetic resonance imaging (MRI) scan: MRI scans use radio waves and strong magnets instead of $X$-rays. Different types of tissue and certain diseased tissue absorb energy from the radio waves, which are transferred into distinct patterns. A computer then translates these patterns into a very detailed image of the suspected tumour. During this process a contrast material called gadolinium is injected intravenously for a clear vision of the tumour. ${ }^{21}$ MRI scans are often the best and most reliable choice of imaging test to investigate a primary bone tumour. 8,12 It accurately determines the intraosseous location and extent of the tumour. ${ }^{12} \mathrm{MRI}$ scans are more uncomfortable than $\mathrm{CT}$ scans and restrict movement for a longer period. ${ }^{8}$

Radionuclide bone scan: In addition to the CT scan and MRI scan, the other type of radiographic test is the radionuclide bone scan. ${ }^{12} \mathrm{~A}$ radionuclide bone scan involves the intravenous infusion of 20 $\mathrm{mCi}$ (millicurie) of technetium 99m methylene diphosphonate (Tc99m MDP) (also called Tc99 MDP bone scan).12,13 The amount of radioactivity used is very low and causes no long-term effects. Following the infusion, images are taken by a dual headed gamma camera, known as bone gammagraphy, at different intervals - "(1) the flow phase, (2) the immediate or equilibrium phase, and (3) the delayed phase. The flow phase demonstrates blood flow, the equilibrium phase shows the reactive vascular flow and the distribution in the intercellular spaces and the delayed phase shows when the radionuclide is excreted in the areas of the osteoblastic activities". ${ }^{13(\mathrm{p} 2)}$ Such osteoblastic active areas are cited on the bone scan image as dense, grey to black areas, called "hot spots." Hence, this bone scan measures the abnormal bone metabolism and further detects any other primary tumour on the same bone or distant bone metastases. ${ }^{12}$ It also provides information on how much damage the primary bone cancer has caused in the bone. ${ }^{8,12}$ This scanning technique is less expensive than other scanning techniques and useful in detecting osseous metastatic osteosarcoma. 8,13

Positron emission tomography (PET) scan: PET scans use fluorodeoxyglucose that contains a radioactive fluorine atom to trace the glucose metabolism. The suspected osteosarcoma cell absorbs this radioactive sugar because of their high rate of metabolism and thereby provides extremely short lived images. PET scans are helpful, as stated: "It is utilized in (1) selecting the region of a tumor most likely to yield diagnostic information for biopsy, (2) staging known malignancies, (3) monitoring the effect of therapy, (4) to establish the cause of suspected recurrence seen on other imaging modalities. It differentiates between fibrosis and recurrent tumor, (5) detecting tumor recurrence, especially in the presence of elevated levels of tumor markers, (6) differentiating benign from malignant lesions, (7) searching for an unknown primary tumor with metastasis of unknown origin, 
(8) guiding radiation therapy planning". ${ }^{13(\mathrm{p} 2)}$ PET scans can combine the images from CT and MRI scans, along with the images of metabolic activity of PET to give a better correlation with biopsy. ${ }^{13}$

\section{Biopsy}

Although diagnosis of osteosarcoma can be made by imaging tests based on the appearance and location of the suspected tumour, it is necessary to have a biopsy for pathological confirmation. ${ }^{12}$ Biopsy is a procedure in which a sample of tissue is taken from the suspected tumour for pathological examination. If cancer is present, the biopsy can confirm whether it is a primary bone cancer or metastatic bone cancer. ${ }^{19}$ There are two types of diagnostic biopsies - closed and open.21 The closed (percutaneous or puncture) biopsy ${ }^{21}$ is known as needle biopsy. The opened (surgical or incisional) biopsy21 is known as surgical bone biopsy. Open biopsy is considered a reliable technique, when more than an adequate amount of sample is required for histological examination and biologic studies. ${ }^{18,19}$ However, the most preferred procedure is closed biopsy. ${ }^{8}$

Needle biopsy: There are two types of needle biopsies - fine needle biopsy' ${ }^{19}$ and true-cut needle biopsy. ${ }^{19}$ For both types, a local anaesthetic is first used to numb the area for the biopsy. Fine needle biopsies use a very thin needle attached to a syringe to withdraw a small amount of sample tissue from the area of the tumour. When the tumour is deeply located, this same needle is used to guide a CT scan. This is also called a CT guided needle biopsy. The true-cut needle biopsy uses a larger needle to extract a sufficient sample for histological examination. ${ }^{19}$ The true-cut needle biopsy is the most considered biopsy when it comes to investigating primary bone cancer. ${ }^{19}$

Surgical bone biopsy: In a surgical bone biopsy, an adequate amount of tissue from the suspected area of tumour is extracted by an expert surgeon for histological examination during which the patient receives general anaesthesia or a nerve block, which numbs a large area.

\section{Laboratory tests}

Tumour samples removed by biopsy are sent for pathological confirmation. ${ }^{18}$ Upon diagnosis of osteosarcoma, the pathologist assigns a grade, which in turn measures the growth and spread of osteosarcoma, following which, blood tests are performed at different intervals: at the time of diagnosis, during systemic treatment and at subsequent follow-up. Blood tests include haemogram values, ${ }^{21}$ biochemical indicators, ${ }^{21}$ alkaline phosphatase, ${ }^{8,21}$ lactate dehydrogenase (LDH), ${ }^{8,21}$ and ionogram data ${ }^{21}$ or rate of red blood cell sedimentation. ${ }^{8}$ Only LDH values can suggest that the osteosarcoma may be more advanced than it appears. ${ }^{8}$ Other laboratory tests include a complete and differential blood count, blood group typing, a coagulation profile, serum electrolytes (magnesium and phosphate), and renal and liver function tests. ${ }^{23}$

\section{Risk factors for osteosarcoma}

Different cancers have different risk factors. The major risk factors for osteosarcoma include age, gender, history of radiation exposure, and inherited cancer syndromes and bone diseases. Osteosarcoma is diagnosed in approximately 1000 new individuals each year in Europe and in a similar number in the United States of America. ${ }^{28,29}$ The prevalence of osteosarcoma in Australia is 
considered to be extremely low; about 195 Australians are diagnosed with osteosarcoma each year. ${ }^{30}$

\section{Age}

The risk of developing osteosarcoma is highest between 10 and 30 years of age. In children and young adults, osteosarcoma usually develops in the areas of rapid bone growth such as the metaphyseal portion, near the ends of long bones, ${ }^{8-10,25,31}$ and around the knee region ${ }^{23}$ of the distal femur or proximal tibia or proximal humerus. ${ }^{14,20,32,33}$ The risk decreases in middle age, but rises again in older adults (usually over the age of 60 ).11,22,34 Osteosarcoma is extremely rare in children below five years. ${ }^{12}$ The incidence rate of osteosarcoma for all age groups is about one in every 10,000 individuals worldwide, of all cancers. ${ }^{35}$

\section{Gender}

The incidence of osteosarcoma at diagnosis is $40 \%$ more in males than females. ${ }^{12,20,23}$

\section{History of radiation exposure}

Adults aged over 60 years who develop osteosarcoma often have other predisposing factors such as a history of radiation exposure ${ }^{19}$ and germline alteration, causing hereditary disorders. The chances of developing osteosarcoma become higher in individuals with a past history of radiation therapy. ${ }^{19}$ Radiation alone can cause up to $3 \%$ of osteosarcomas. ${ }^{5}$ Additionally, the imaging tests that use different types of radiation may also add to the risk of developing osteosarcoma. ${ }^{5}$

\section{Inherited cancer syndromes and bone diseases}

There are certain inherited cancer syndromes that may lead to the risk of developing osteosarcoma. This may include the following syndromes as listed below.

1) Retinoblastoma of the eye caused by a mutation in the $p R b$ gene ${ }^{12,14}$ which is treated by radiation therapy. There is an increased risk of developing osteosarcoma in bones around the eyes (and nearby). ${ }^{9}$

2) Li-Fraumeni syndrome which is usually caused by a germline deletion of the p53 tumour suppressor gene. ${ }^{12,14}$ Up to $12 \%$ of patients with this syndrome are likely to develop osteosarcoma. $^{5}$

3) Rothmund-Thompson, mainly seen in children, caused by abnormal changes in the RECQL4 gene, who tend to have short stature, skeletal problems and rashes. ${ }^{5}$

4) Bloom syndrome (BLM), caused by mutation in BLM $3^{\prime} \rightarrow 5^{\prime}$ DNA helicase of RecQ family. Up to $3 \%$ of patients develop osteosarcoma. ${ }^{5}$

5) Werner syndrome (WRN), called progeria, caused by mutations in WRN helicase RECQL2. Up to $10 \%$ of patients have increased chances of developing osteosarcoma. ${ }^{5}$

6) Certain bone diseases may also lead to osteosarcoma, such as Paget disease of the bone, a benign condition characterised by abnormal development of new bone cells ${ }^{29}$ that mainly develops in the adults aged over 60 years. 


\section{Current treatment regimen for high-grade osteosarcoma}

The standard treatment of care for high-grade non-metastatic and metastatic osteosarcoma involves neoadjuvant multi-agent chemotherapy, followed by surgical resection of the tumour and adjuvant multi-agent conventional chemotherapy. 15,25-27,31-34,36,37

\section{Chemotherapy}

Since the 1970s, the gold standard treatment for patients with osteosarcoma is chemotherapy. ${ }^{14}$ The principle standard protocol for multi-agent chemotherapy ${ }^{14,15,23,26,32,33,36}$ comprises cisplatin, doxorubicin (adriamycin), high-dose methotrexate with leucovorin rescue, and ifosfamide, all of which have both acute and long-term toxicity. Initial baseline evaluation includes renal function (with a creatinine clearance determination), an echocardiogram or a radionuclide (for doxorubicin that can cause cardiac toxicity) and an audiogram (for cisplatin that can cause deafness). 18,19,23,31 Neoadjuvant chemotherapy is administered for a period of about eight to 10 weeks. ${ }^{22}$ This preoperative chemotherapeutic treatment offers time to achieve tumour shrinkage and induces tumour necrosis in the primary tumour to assist in resection of the tumour. ${ }^{22} \mathrm{It}$ also offers time to study the histological effect of preoperative chemotherapy on the primary tumour to possibly alter postoperative chemotherapy. The degree of tumour necrosis is a prognostic marker used to validate the effectiveness of neoadjuvant chemotherapy treatment.

\section{Surgery}

Currently, surgery remains an essential part of osteosarcoma treatment along with chemotherapy. Following neoadjuvant chemotherapy, surgical control of the primary tumour is achieved by surgical resection of macroscopically detected tumour using radical surgery. The surgical treatment of osteosarcoma consists of various options such as amputation (of at least one bone above an affected limb), ${ }^{18,34}$ limb salvage with endoprosthetic or biological reconstruction, and rotationplasty. ${ }^{34}$ The choice of these options depends on tumour grade, location, response to neoadjuvant chemotherapy, patient's preference and functional outcome. A wide margin of normal tissue resection of the primary tumour is an essential aspect of surgical treatment of osteosarcoma to avoid local recurrence and improve overall survival. ${ }^{34}$ With this rationale, and the observation of the neoadjuvant chemotherapy, postoperative adjuvant chemotherapy is continued for a period of 12-29 weeks. ${ }^{22}$

Current treatment for osteosarcoma achieves $60-70 \%$ five-year event-free survival (EFS) in nonmetastatic osteosarcoma patients 27 and approximately 20\% five-year EFS in metastatic osteosarcoma patients. Although, the use of adjuvant chemotherapy has been shown to improve tumour control and patients survival, the exact role of adjuvant chemotherapy is unclear. ${ }^{36}$

However osteosarcoma remains fatal in many patients. ${ }^{12}$ To improve the survival rates of these patients, novel forms of therapy are needed. One of the major drawbacks in the treatment of osteosarcoma is chemoresistance. The onsets of micro-metastatic deposits have shown resistance to postoperative/adjuvant chemotherapy and require a comprehensive understanding of the mechanisms of resistance involved against multiagent chemotherapy. ${ }^{15}$ 


\section{Innovative therapeutic approaches}

The existing chemotherapy regimen have achieved desirable efficacy in patients showing good histological responses, but it has failed to show the expected therapeutic effects in patients with poor responses to chemotherapy..$^{15}$ There is a critical need for new effective therapies such as immune stimulating agents against chemotherapy-resistant tumours for the treatment of osteosarcoma. ${ }^{38}$ These innovative therapeutic approaches ${ }^{37,39}$ may be used in combination with existing multiagent chemotherapy in an adjuvant chemotherapy setting. ${ }^{40}$ This may aid in preventing tumour recurrence, provide a superior anti-tumour effect, and improve the survival rates in osteosarcoma patients. ${ }^{41}$

\section{Immune stimulatory agents}

Since the 1980s, advances in systemic chemotherapy has improved the outcomes of non-metastatic osteosarcoma patients, however, there has been no altered improvement in the survival of patients with metastatic osteosarcoma. ${ }^{38}$ This has led to novel biologic therapeutic approaches, including immunotherapy. Immunotherapy is the use of cytokines or immunostimulatory agents. In the past, investigations with immunotherapy have had little or no impact on patients with primary bone cancer. Furthermore, there is an urgent need for well-designed, multi-institutional clinical trials to recommend or further refute any conclusions regarding the application of immunotherapy for the treatment of osteosarcoma. Many clinical trials based on other immune modulation strategies have been investigated in the context of osteosarcoma. This has included antigens that are specifically expressed on osteosarcoma tumours such as therapeutic antibody administration (abbreviated to TP3), human epidermal growth factor receptor-2 (abbreviated to HER2), insulin-like growth factor receptor 1 (abbreviated to IGF1R), cytokine/immune administration (systemic interferon or inhaled granulocyte macrophage colony stimulating factor), and vaccination strategies (dendritic cells exposed to osteosarcoma total RNA). ${ }^{37,39} \mathrm{All}$ of these have elicited a similar response with promising results and limited clinical application, however, the different mechanisms of action are unclear. Despite these immune modulation strategies, escalation of immune responses with immune stimulatory agents continues to be of interest at present in the treatment of high-grade osteosarcoma. $39,42,43$

\section{Mifamurtide drug history}

Among the most recently investigated immune stimulatory agents is mifamurtide. Mifamurtide (also known as muramyl tripeptide [MTP] and muramyl tripeptide phosphatidylethanolamine [MTP$\mathrm{PE}]^{25,44,45}$ is a fully synthetic lipophilic analogue of muramyl dipeptide (MDP), ${ }^{42}$ the smallest naturally-occurring immune stimulatory component of bacterial cell walls. ${ }^{31,40,46-52}$ MTP-PE results from the covalent addition of alanine and dipalmitoyl phosphatidyl ethanolamine to MTP. ${ }^{10,47}$ Mifamurtide was originally developed by Novartis (formerly CIBA-Geigy) and is currently developed by IDM Pharma for osteosarcoma. 45

\section{The molecule and its formulation}

There are two formulations of mifamurtide in use, the free-drug form (MTP-PE) and the liposomal encapsulated form, liposomal MTP-PE (L-MTP-PE).50 L-MTP-PE is specifically designed to 
selectively deliver the drug to macrophages and monocytes to activate them to be tumouricidal. ${ }^{31,48,50,53}$ Furthermore, L-MTP-PE has a very rapid clearance from the blood with no accumulation of phospholipid following repeated administration. ${ }^{16}$ Hence, L-MTP-PE is 10 times less toxic than MTP-PE. ${ }^{17,50}$ One study ${ }^{2}$, which was the largest ever conducted randomised controlled trial, found that the addition of L-MTP-PE to standard chemotherapy appeared to lead to an improvement in six-year overall survival, from $70 \%$ to $78 \%$ for non-metastatic osteosarcoma, and reduce the risk of death from osteosarcoma by one third.

\section{Mechanism of action}

Mifamurtide stimulates immune responses via binding to nucleotide-binding oligomerisation domaincontaining protein 2 (NOD2), an intracellular pattern-recognition receptor molecule expressed mainly in monocytes, macrophages and dendritic cells. ${ }^{17,47}$ By binding to NOD2, mifamurtide activates the nuclear factor (NF)-KB pathway that leads to an increased production of proinflammatory cytokines such as tumour necrosis factor-alpha (TNF-a), interleukin 1 (IL-1), interleukin 6 (IL-6), interleukin 8 (IL-8), interferon gamma (IFN-gamma), and immune stimulation markers plasma neopterin and serum C-reactive protein. ${ }^{17,48}$ MTP-PE being lipophilic in nature is directly incorporated into the lipid bilayer structure of a liposome ${ }^{54}$ because of which, any mifamurtide leakage is eliminated, ${ }^{54}$ and further to this process, macrophages and monocytes phagocytose mifamurtide kills tumour cells and not the normal cells. ${ }^{32,55}$ Activation of these tumouricidal macrophages and monocytes may have the potential to eradicate residual micrometastases that exist in osteosarcoma patients after removal of the primary tumour, which is otherwise resistant to postoperative adjuvant chemotherapy. ${ }^{54,56,57}$ Furthermore, the immunostimulatory effects of mifamurtide are similar to MDP with the advantage of a longer half-life in plasma with less pyrogenic effects. Within the scope of a clinical trial, mifamurtide did show its maximum effect against minimal residual micrometastases and was further included as a standard part of treatment with adjuvant chemotherapy following surgical resection of the primary tumour. ${ }^{56}$ Stimulation of the innate immune system by mifamurtide can be measured by: (1) analysis of blood plasma cytokine levels ${ }^{57}$ such as rapid induction of circulating TNF-a $a^{50,58,59}$ and IL-650,58,59 (one to two hours postinfusion); (2) prolonged elevations in plasma neopterin ${ }^{50,58,59}$ (24 hours postinfusion) and serum C-reactive protein ${ }^{58,59}$ (72 hours postinfusion); and (3) fibrosis ${ }^{57}$ of the area from which the tumour is removed, inflammatory macrophage infiltration into the lesions, and change in the histological characteristic ${ }^{57}$ of the tumour from high-grade to low-grade.

\section{Dosage and administration of mifamurtide}

L-MTP-PE is supplied as a lyophilised product in glass vials containing $4 \mathrm{mg}$ of the active biological agent stored at $4^{\circ} \mathrm{C}$ until use, together with a filter and instructions for preparation of the liposomal infusion suspension. ${ }^{16,60}$ The reconstitution involves the addition of $0.9 \% \mathrm{NaCl}$ to the lyophilisate that is warmed to room temperature over 30 minutes allowing it to hydrate, followed by vigorous shaking for one minute to allow liposome formation. 60 The reconstituted liposomes are withdrawn through a filter and then transferred to an infusion bag containing additional saline. The maximum tolerated dose (MTD) of mifamurtide is $6 \mathrm{mg} / \mathrm{m}^{2}$ with a moderate toxicity that has some dose-limiting side effects such as chills, fever, malaise and nausea, ${ }^{51}$ while the optimal biological dose is $2 \mathrm{mg} / \mathrm{m}^{2}$ for the activation of macrophage and monocyte cytotoxicity. ${ }^{40,57}$ The standard dosing schedule 
recommended for delivery of both forms of mifamurtide is $2 \mathrm{mg} / \mathrm{m}^{2}$ infused intravenously over a onehour period twice a week for 12 weeks, then once a week for 12-24 weeks for a total of 36 weeks and 48 doses. $10,25,31,40,50,55,58-60$

This chapter introduced the topic of interest, osteosarcoma and a new treatment strategy with an immune stimulatory agent that warrants further investigation in the form of a systematic review.

The next chapter provides an introduction to evidence-based healthcare and the methodology used in systematic reviews. It further addresses the need for conducting a systematic review on this topic. 


\section{CHAPTER 2: METHODOLOGY}

This chapter of the thesis introduces the methodology used in a systematic review. It starts with an introduction to evidence-based healthcare and the Joanna Briggs Institute (JBI), including its approach to evidence-based healthcare. Following this is a discussion on evidence synthesis and the systematic review, Levels of Evidence, and the GRADE (Grading of Recommendations Assessment, Development and Evaluation) approach. Finally, the need for conducting a review in this area is proposed.

\section{Evidence-based healthcare (EBHC)}

Use of the best available evidence to assist in clinical decision-making regarding the care of individual patients or the delivery of health services is the foundational basis of evidence-based healthcare. Ideally, within the healthcare system, all clinical decisions should be based on the best available evidence, the individual patient preference, the context in which care is delivered, and the professional judgement and expertise of the individual health professional. ${ }^{61}$ Hence, evidence plays a major role in clinical decision-making.

Evidence can be defined "as the basis of belief; the substantiation or confirmation that is needed in order to believe something is true". ${ }^{61(210)}$ Evidence generated by research is the most reliable type of evidence and can be quantitative or qualitative in nature. Quantitative research produces numerical data by using traditional scientific methods that seek to establish relationships between two or more variables. ${ }^{62}$ Common quantitative study designs include randomised controlled trials (RCTs), nonrandomised controlled trials, cohort studies, cross sectional studies, case series and case reports. Qualitative research focuses on human experiences and cultural and social phenomena. ${ }^{62}$ There are a range of qualitative research methods including ethnography, phenomenology, qualitative inquiry, action research, discourse analysis and grounded theory. ${ }^{62}$ While both are important, this thesis focuses on quantitative evidence.

The concept of evidence-based practice originated in the mid-19th century in Paris where Pierre Charles Alexandre Louis undertook the first known experiment/evaluation regarding bloodletting for the treatment of pneumonia. ${ }^{63,64}$ This was followed by the work of James Lind who performed the first clinical trial, looking at the cause of scurvy amongst British sailors and how to treat it with the use of oranges and lemons. ${ }^{64}$ According to Sackett et al., EBHC is "the conscientious, explicit and judicious use of current best evidence in making decisions about the care of individual patients". ${ }^{63(p 71)}$ A number of models have been proposed to explain the process of EBHC. 65 Despite the developments in EBHC, some critics refer to it as being "old hat", "cookbook" medicine that may be impossible to practice, and possibly leading to cost cutting. ${ }^{63}$ However, Sackett et al. argues that "a bottom up approach is required that integrates the best external evidence with individual clinical expertise and the patient's choice. External clinical evidence can inform, but can never replace, individual clinical expertise, and it is this expertise that decides whether the external evidence applies to the individual patient at all, and if so, how it should be integrated into a clinical decision".63(p72) Additionally, another criticism of the evidence-based approach is the emphasis on 
the systematic reviews of evidence of effectiveness and the meta-analysis of RCTs. ${ }^{66}$ Traditionally, RCTs have been the highest form of evidence and the best approach to generate evidence of effectiveness. There is an ongoing debate in the literature about creating a balance to value both quantitative and qualitative research.66 JBI adopts a more inclusive approach to what constitutes evidence, where the "findings of qualitative research studies and other text derived from opinion, experience and expertise are acknowledged as forms of evidence". 61 (p211)

\section{The JBI approach to EBHC}

The JBI Model of EBHC (referred to hereafter as the 'JBI Model') was first published in $2005 .{ }^{65}$ It is based on the Institute's approach to translating the best available evidence into best practice in the appropriate healthcare setting. ${ }^{65} \mathrm{JBI}$ considers the best available evidence as evidence that may arise from published and unpublished studies that is inclusive of diverse sources of research-based and non-research-based evidence. ${ }^{67}$ The process of EBHC requires the generation, dissemination, implementation and evaluation of evidence-based guidelines to improve global health. ${ }^{61}$

The JBI Model demonstrates the importance of research conducted which is based on concerns raised by health carers to address a specific question. ${ }^{61}$ However, in instances where research does not exist, clinicians are still required to make decisions about care and to provide the most meaningful and useful information to inform their decision-making. EBHC seeks evidence from the literature to answer questions on the feasibility, appropriateness, meaningfulness and effectiveness of a specific intervention for a particular condition. 65 The JBI Model was recently updated in 2016 and is presented in Figure 1.65

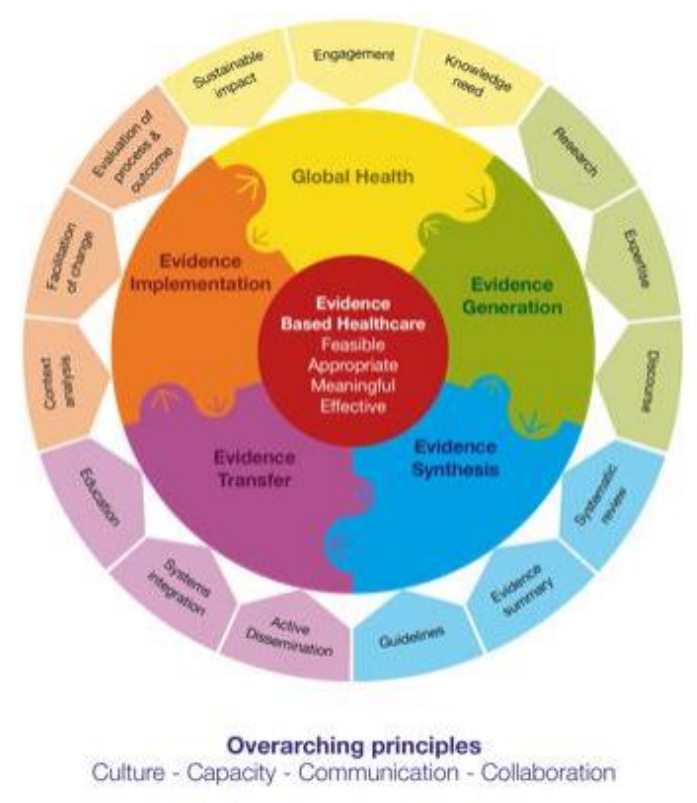

Figure 1: The JBI Model65

The central circle of the model represents the 'Pebble of Knowledge', a conceptualisation of EBHC, which relates to the Feasibility, Appropriateness, Meaningfulness and Effectiveness (FAME) scale. ${ }^{65}$ 
Feasibility relates to the extent to which an activity/intervention is viable to occur in a particular setting/context, appropriateness refers to the context of care given during an activity/intervention while meaningfulness relates to the patient experience. Lastly effectiveness relates to the level an activity/intervention is able to achieve its intended clinical outcome. ${ }^{61,65}$ The Inner Wedges depict five component parts of the JBI Model: global health, evidence generation, evidence synthesis, evidence transfer and evidence implementation. ${ }^{65}$ Lastly, the Outer Wedges depict the operational components of the JBI Model. For global, health there are three major components - sustainable impact, engagement and knowledge need. For evidence generation, discourse, experience and research are proposed. For the evidence synthesis wedge, three components are suggested systematic reviews, evidence summaries and clinical guidelines. The evidence transfer wedge encompasses active dissemination, education programs and systems integration. Lastly, the evidence implementation wedge includes context analysis, facilitation of change, and evaluation of process and outcome. This thesis sits within the evidence synthesis wedge as it involves the conduct of a systematic review on the effectiveness of mifamurtide in addition to standard chemotherapy for high-grade osteosarcoma.

\section{Evidence synthesis}

The JBI Model defines evidence synthesis as "the evaluation or analysis of research evidence and opinion on a specific topic to aid in decision making in healthcare". ${ }^{61(p .211)}$ To inform practice, a review of all relevant evidence needs to be undertaken; the results are then collated into and displayed in a usable format. Fourteen different types of reviews ${ }^{68}$ have so far been identified. The most popular being literature reviews, scoping reviews and systematic reviews. ${ }^{68,69}$ Literature reviews typically include only published studies, may or may not include comprehensive searching, and may or may not include a critical appraisal assessment. The synthesis is characteristically provided in narrative form, and interpretations may be presented theoretically. A scoping review is often used to inform systematic reviews by identifying research gaps and summarising findings of research. ${ }^{70}$ Scoping reviews identify a research question and relevant studies but do not include a formal assessment of included studies, limiting data synthesis and interpretation. ${ }^{69,70}$ Systematic reviews remain the core of evidence synthesis; they are essentially a summary of knowledge and collation of findings about a specified question.

The synthesis of the results of quantitative research depends on the nature of the included studies in the review. Quantitative systematic reviews based on the results of RCTs were historically seen as the core of systematic reviews, however, as described above, what constitutes as evidence and the types of questions people want answered have evolved. ${ }^{61}$ Different questions require different kinds of research. ${ }^{66}$ 


\section{The systematic review}

A systematic review by definition is "...the objective, transparent and unbiased location and critical appraisal of the complete scope of research in a given topic and the eventual impartial synthesis and, if possible, meta-analysis of individual study findings. Therefore, in order to address a specific research aim, a systematic review collates all evidence that fits pre-specified eligibility criteria". ${ }^{11(p 71)}$

Depending on the nature of the question, systematic reviews may consider different types of evidence: quantitative, qualitative, an evaluation of health economics, or textual evidence. ${ }^{67} \mathrm{~A}$ systematic review that considers at least two different types of evidence (such as qualitative and quantitative) in order to address a particular review question is called a mixed methods review.62,69 It is generally accepted that systematic reviews follow seven steps:

1. Research question: The first step of a systematic review is formulating a research question which summarises the objective of the review. ${ }^{62}$ The research question identifies the inclusion criteria for considering studies and should make reference to the review's intended population, intervention, comparator and outcomes (PICO) (for quantitative reviews). ${ }^{62}$

2. Research protocol: Following the research question, the research protocol is developed which is usually subjected to peer review prior to commencing the review.62 The main objective of a research protocol is to develop methods for literature searching, data extraction and data synthesis before starting. ${ }^{62} \mathrm{~A}$ requirement of a JBI systematic review is an a priori published protocol. 72

3. Comprehensive search strategy: The literature search aims for exhaustive, comprehensive searching 68 to identify all international research relating to the review question. ${ }^{62}$ The JBI approach aims to identify both published and unpublished studies utilising a three-stage search process. ${ }^{62}$

4. Critical appraisal: The aim of a systematic review is to synthesise the best available evidence, hence the methodological quality of included studies needs to be appraised using validated checklists or tools to assess for biases. ${ }^{62}$ Quality assessment is undertaken by two independent reviewers to determine inclusion/exclusion of studies. ${ }^{68}$

5. Data extraction: Details regarding participants, interventions, comparators and outcomes are extracted from included studies. ${ }^{62}$ Use of a standardised extraction tool aims to minimise errors in extracting data.

6. Data synthesis: This involves the analysis of results. It is important to combine study data only when it is appropriate to do so, otherwise the analysis and subsequent conclusions drawn may not be valid.62 Interventions, comparators and outcomes are examined to identify research that can be appropriately combined to enable the results of similar studies to be pooled, where possible, to perform a meta-analysis which permits a summary about the effect size of an intervention compared to a control. The analysis in the review process investigates if combined results provide a more meaningful interpretation of outcome, simply due to the larger power of participant numbers. The method of data synthesis will always influence the findings of a systematic review. Where meta-analysis is appropriate, 
the review should describe the statistical methods and software package (such as the JBI System for the Unified Management, Assessment and Review of Information [JBISUMARI]) being used. However, if the data is found to be heterogeneous, the results are then presented in narrative form, including the reasons of heterogeneity and the inappropriateness of combining the data statistically.

7. Interpretation of results: Data can be analysed and interpreted, including the strengths and weaknesses of the included studies. The method of data synthesis will always influence the findings of a systematic review. Conclusions should be based on the available evidence, and recommendations for practice and future research are provided at the end. ${ }^{6}$

The above seven steps are generally accepted across the systematic review community. As far as the methodological approach is concerned, the Cochrane Collaboration ${ }^{73}$ and the $\mathrm{JBI}$ methodology are predominantly used to conduct a systematic review to assess quantitative evidence for the effectiveness of an intervention. ${ }^{62}$ Systematic reviews are often referred to when decisions are implemented in practice. However, systematic reviews can have their limitations which include addressing a narrow clinical question and the length of time they take to complete (typically between six months and two years), and are thus very expensive.

\section{Levels of evidence and the grading of recommendations}

It is necessary to rank evidence as it varies in quality in terms of methods used. There are a variety of evidence hierarchies available; however, the JBI classification system for ranking the levels of evidence incorporates a wide range of research methodologies, including effectiveness, diagnosis, prognosis, economic evaluations and meaningfulness (Appendix I). Systematic reviews are considered to be the highest level of evidence. ${ }^{73}$ Of the included studies of this systematic review, one study (based on two papers) 1,2 was classified as level 1.c and the other ${ }^{3}$ was classified as level 2.d primary studies of effectiveness.

In 2014, the GRADE (Grading of Recommendations, Assessment, Development and Evaluation) approach to grade quality of evidence and strength of recommendations was developed. ${ }^{74}$ This approach assists in collating the results of quantitative research. It considers issues such as risk of bias, publication bias, inconsistency, indirectness, imprecision of evidence, effect sizes, doseresponse relationships and confounders of findings (Table 2). ${ }^{74}$ The evidence included in a systematic review is given a rank out of four possible levels (High, Moderate, Low and Very Low). The evidence is first assigned a pre-rank based on its study design: high quality for RCTs and low quality for observational studies. The evidence can then be downgraded or upgraded based on the above mentioned factors. Factors that lead to downgrading include risk of bias, inconsistency, indirectness of evidence, imprecision of results and publication bias. ${ }^{74}$ Factors that lead to upgrading include large magnitude of effect, dose response and plausible confounding factors. ${ }^{74}$

JBI follows the GRADE approach to establish the confidence in synthesised findings pertaining to systematic reviews of effectiveness. This GRADE approach recommends the development of 'A Summary of Findings' table, which can be created from the GRADEpro website (http://gradepro.org). 
Table 2: Application of GRADE quality of evidence in the GRADE approach

\begin{tabular}{|l|l|}
\hline Quality & Rating quality of results/findings \\
\hline Risk of bias & $\begin{array}{l}\text { Assessed based on the limitations in study design to downgrade the quality of } \\
\text { evidence for the outcome into: } \\
\text { (a) not serious (b) serious (c) very serious }\end{array}$ \\
\hline Inconsistency & $\begin{array}{l}\text { Assessed based on the results being consistent enough across the studies to } \\
\text { downgrade the quality of evidence into: } \\
\text { (a) not serious (b) serious (c) very serious }\end{array}$ \\
\hline Indirectness & $\begin{array}{l}\text { Assessed based on whether the evidence answers directly the health care } \\
\text { question asked being enough to downgrade the quality of evidence for the } \\
\text { outcome into: } \\
\text { (a)not serious (b) serious (c) very serious }\end{array}$ \\
\hline Imprecision & $\begin{array}{l}\text { Assessed based on the results being precise or not enough to downgrade the } \\
\text { quality of evidence for the outcome into: } \\
\text { (a) not serious (b) serious (c) very serious }\end{array}$ \\
\hline Publication bias & $\begin{array}{l}\text { Assessed based on the probability of publication bias serious enough to } \\
\text { downgrade the quality of evidence for the outcome into: (a) undetected (b) } \\
\text { strongly suspected }\end{array}$ \\
\hline Large effect & $\begin{array}{l}\text { Assessed based on the magnitude of effect being large or very large, and if so to } \\
\text { upgrade the quality of evidence for the outcome into: (a) no (b) large (c) very } \\
\text { large }\end{array}$ \\
\hline Plausible confounding & $\begin{array}{l}\text { Assessed based on the evidence found of studies that the influence of all plausible } \\
\text { residual confounding would reduce a demonstrated effect or suggest a spurious } \\
\text { effect to either downgrade or upgrade the quality of evidence for the outcome into: } \\
\text { (a)no (b) would reduce a demonstrated effect (c) would suggest a spurious } \\
\text { effect }\end{array}$ \\
\hline $\begin{array}{l}\text { Assessed based on the presence of evidence of dose-response gradient upgrade } \\
\text { the quality of evidence for the outcome into: } \\
\text { (a) no (b) yes }\end{array}$ \\
\hline
\end{tabular}

The JBI Grades of Recommendation were developed to assist healthcare professionals when implementing evidence into practice. ${ }^{75}$ Essentially, there are two grades of recommendation, Grade A - a strong recommendation for a specific intervention and Grade B - a weak recommendation for a specific intervention (see Appendix II). This approach looks at: (1) whether desirable effects outweigh undesirable effects, (2) whether there is evidence of adequate quality that may support its use, (3) whether there is a benefit or no impact on resource use, and (4) whether values, preferences and the patient experience have been taken into account. ${ }^{75}$

\section{The need for conducting a review}

A systematic review is the first step in guideline development and is a tool for improving health outcomes, by combining outcomes of primary studies, and also identifying gaps in existing evidence. It provides information on what is known or not known, and can potentially shed further light on when further research is required.

In this thesis, and the systematic review that underpins it, the effectiveness of mifamurtide in addition to standard chemotherapy compared to the standard chemotherapy was examined on selected health outcomes of osteosarcoma patients using JBI methodology. In order to gain better understanding of the micro-metastatic deposits, which are resistant to postoperative/adjuvant chemotherapy, a review of alternate therapeutic approaches with an immune stimulatory agent, such 
as mifamurtide, is required. No such systematic reviews have been conducted relating to treatment of osteosarcoma patients with mifamurtide in the adjuvant setting. Chapter 3 outlines the systematic review methods including eligibility criteria, search strategy, study selection, critical appraisal, data extraction and data synthesis methods. 


\section{CHAPTER 3: SYSTEMATIC REVIEW METHODS}

Chapter 3 outlines the systematic review methods. This covers the review eligibility criteria, search strategy, study selection process, including how studies were critically appraised, data extraction and data synthesis methods. Prior to undertaking the current systematic review, a preliminary search of the JBI Database of Systematic Reviews and Implementation Reports (JBISRIR), PubMed and the Cochrane Library was undertaken to ascertain the suitability of the topic and to determine if this or a similar systematic review had already been conducted. ${ }^{72}$ It was found that no other systematic review on this topic, evaluating the effectiveness of mifamurtide as an adjunct to chemotherapy for high-grade, resectable, non-metastatic and metastatic osteosarcoma, was located. The objectives, inclusion criteria and methods of analysis for this review were specified in advance and documented in the protocol which has been published in the JBISRIR. ${ }^{72}$

\section{Inclusion criteria}

\section{Types of participants}

This review included patients with high-grade, resectable, non-metastatic and metastatic osteosarcoma based on histological diagnosis. Patients with other types of bone cancer such as Ewing's sarcoma and chondrosarcoma were excluded from this review.

\section{Types of interventions and comparator}

This review considered studies that evaluated intravenous infusion of either of the pharmaceutical formulations of mifamurtide (MTP-PE or L-MTP-PE) in addition to standard multi-drug chemotherapy (such as any combinations of cisplatin, doxorubicin, high-dose methotrexate, and ifosfamide) after macroscopically complete surgical resection. The current recommended dose for delivery of mifamurtide is $2 \mathrm{mg} / \mathrm{m}^{2}$ administered as an intravenous infusion over a one-hour period twice a week for 12 weeks, with dosing at least three days apart, then once a week for 12-24 weeks.1,2 Only studies that complied with this regimen were included in the review. The comparison group for this review were patients who had received standard chemotherapy alone.

\section{Types of outcomes and outcome measures}

This review considered studies that measured at least one of the following primary or secondary outcomes measures:

Primary outcomes:

- Event-free survival (EFS): measured in years post-intervention from study commencement until any recurrence or tumour progression of osteosarcoma.

- Overall survival (OS): measured in percentage of patients surviving post-intervention with the time to follow-up after the treatment up to 10 years post-intervention.

- Recurrence of osteosarcoma: detected by X-ray, computed tomography (CT) scans and magnetic resonance imaging (MRI) scans, tumour markers (such as blood plasma levels for any changes which included: (1) variation in cytokine levels with circulating TNF- $\alpha$ and IL-6; (2) elevation of serum C-reactive proteins and plasma neopterin; and (3) any histological 
reappearance of tumours.

Secondary outcomes:

- Health-related quality of life (HRQoL): as measured using any validated and standardised tool.

- Mifamurtide-related adverse events: measured by any type of classification systems.

\section{Types of studies}

This review considered experimental study designs, including randomised controlled trials, nonrandomised trials, quasi-experimental studies, and before and after studies. In the absence of experimental studies, observational studies were considered.

\section{Review methods}

\section{Search strategy}

The search strategy aimed to find both published and unpublished studies. A three-step search strategy was utilised in this review. An initial limited search of MEDLINE and CINAHL was undertaken followed by an analysis of the text words contained in the title and abstract, and of the index terms used to describe the article. A second search using all identified keywords and index terms was then undertaken across all included databases. Thirdly, the reference lists of all identified reports and articles were searched for additional studies. The search strategies and results for each database are appended (Appendix III).

Only studies published in English from 1990 to June 2016 were considered for inclusion in this review. Even though mifamurtide was synthesised in the 1980s, it was not investigated in clinical trials as a treatment for osteosarcoma until the1990s. ${ }^{26,50}$

The databases searched for published studies were:

- PubMed

- CINAHL

- Embase

- Cochrane Library (CENTRAL)

- Scopus

- Web of Science

- Cancerlit.

The search for unpublished studies included the following searchable databases, and government agency and organisation websites:

- Google advanced search

- MedNar

- Clinicaltrials.gov

- National Library of Medicine

- National Comprehensive Cancer Network (NCCN) website (www.nccn.org)

- National Cancer Institute (NCl) website (www.cancer.gov) 
- CureSearch website (www.curesearch.org).

Initial keywords were:

'osteosarcoma', 'osteosarcoma, juxtacortical', 'osteogenic sarcoma', 'mifamurtide', 'acetylmuramylalanyl-isoglutamine', 'muramyl tripeptide phosphatidylethanolamine', 'L-MTP-PE', 'biological response modifiers', 'muramyl tripeptide'.

\section{Assessment of methodological quality}

Quantitative papers selected for retrieval were assessed by two independent reviewers for methodological validity prior to inclusion in the review using the standardised critical appraisal instrument from the Joanna Briggs Institute Meta Analysis of Statistics Assessment and Review Instrument (JBI MAStARI) (Appendix IV).

\section{Data extraction}

Quantitative data was extracted from papers included in the review using the standardised data extraction tool from JBI MAStARI (Appendix V). The data extracted included specific details about the interventions, populations, study methods and outcomes of significance to the review question and specific objectives.

\section{Data synthesis}

The limited number of identified studies that investigated the effects of mifamurtide, the outcome measures reported and the lack of homogeneity between the populations and interventions prevented any possible meta-analysis. Hence, results from the review are presented in narrative form.

Chapter 4 outlines the search results, reasons for excluded studies, critical appraisal of methodological quality, and an overview of the three papers detailing two studies included in the systematic review. 


\section{CHAPTER 4: RESULTS}

This chapter details the findings of the systematic review conducted to evaluate the effectiveness of mifamurtide in addition to standard chemotherapy for high-grade osteosarcoma. A detailed description of the search results, the study selection process, and the methodological quality for critical appraisal is presented, followed by a description of the included studies. Finally a narrative synthesis of the results organised by outcome is provided.

\section{Description of search results and study selection}

The search results and study selection and inclusion process is outlined in Figure 2. The search yielded 3000 potentially relevant articles, following which, 462 duplicate publications were removed and the remaining 2538 articles were examined for relevance to the review based on title and abstract. After reading the titles and abstracts, 2479 articles were excluded as they did not relate to the review question and subsequent inclusion criteria of this review (e.g. they were non-experimental study designs, did not assess the outcomes of interest to the review, while others were nonrelevant). The remaining 59 articles were retrieved in full text and 15 more papers were identified after reference review. Finally, out of the total 74 articles, 67 did not meet the inclusion criteria and were excluded. Four citations which appeared to meet the inclusion criteria were excluded as three were poster presentation abstracts, for which full texts were unavailable, despite the authors being contacted, and one was a recently published meeting abstract (author contact details unavailable). ${ }^{49,56,76,77}$ Appendix VI provides details of the excluded studies after review of full text and reasons for their exclusion. The remaining three papers were assessed for methodological quality and were included in the review. 

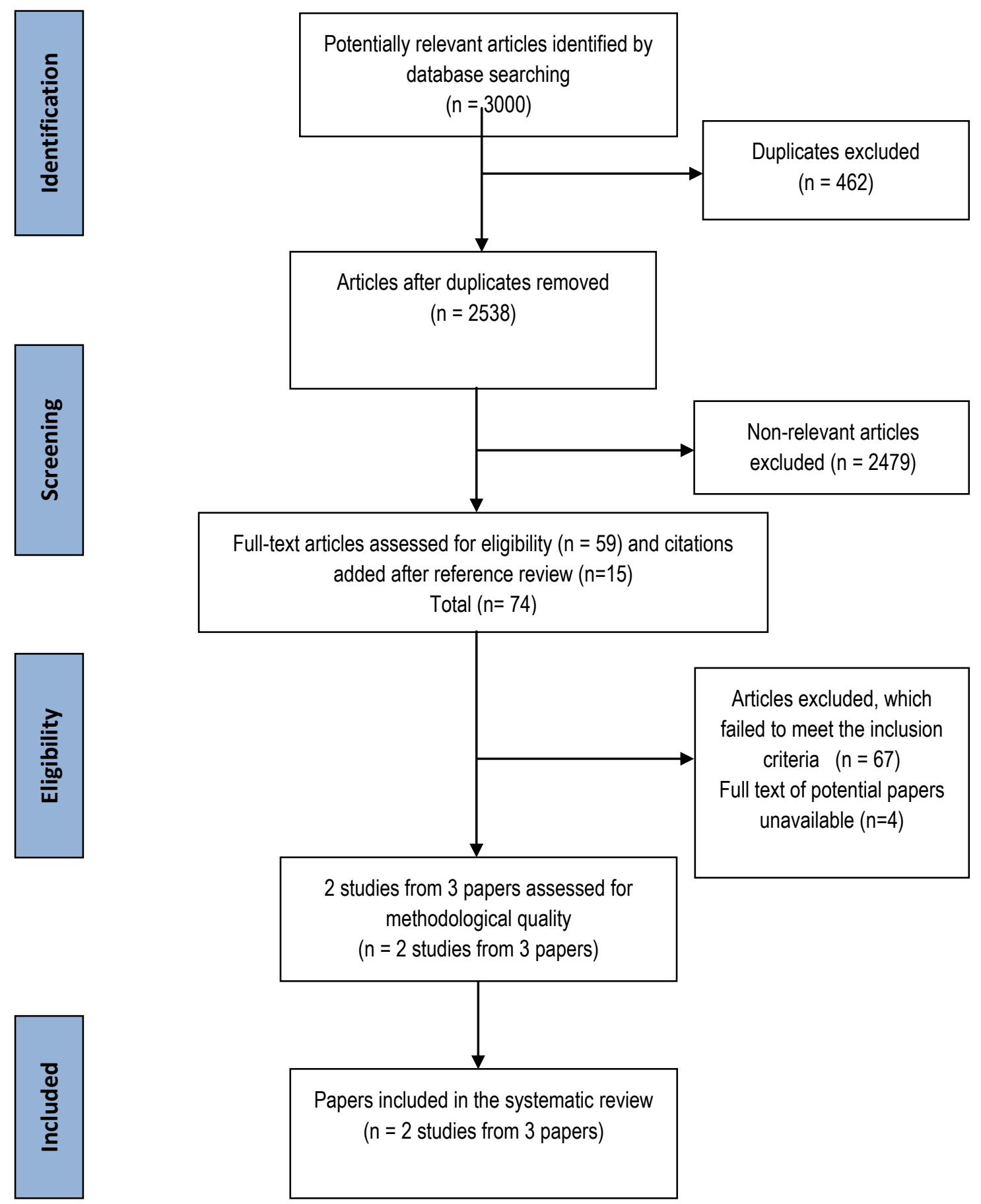

Figure 2: Flow chart for identification of studies for inclusion and exclusion

From: Moher D, Liberati A, Tetzlaff J, Altman DG, The PRISMA Group (2009). Preferred Reporting Items for Systematic Reviews and Meta-Analyses: The PRISMA Statement. PLoS Med 6(6): e1000097. 


\section{Description of included studies}

Three papers detailing two studies were included in the review. ${ }^{1-3}$ Two papers ${ }^{1,2}$ reported on the same RCT and one paper ${ }^{3}$ was a quasi-experimental before and after study. The reviewers agreed that all three papers should be included for completeness and consideration of the best available evidence. All included studies were undertaken in the United States of America (USA). A total of 802 patients were included in the two studies from the three papers. ${ }^{1-3}$ Participants were outpatients, aged one to 70 years, who were diagnosed with histologically confirmed high-grade non-metastatic and/or metastatic or relapsed osteosarcoma. All participants, regardless of age, gender or ethnicity, received the same dose of mifamurtide. None of the studies provided comprehensive details about the size of the hospital or the services provided.

\section{Randomised controlled trial}

Two papers ${ }^{1,2}$ were based on the same research study conducted at different centres, the Children's Cancer Group (CCG) institutions and Children's Oncology Group (COG) institutions, respectively, USA, that carried out Intergroup Study 0133 from November 1993 to November 1997. This was the largest ever completed and published pivotal phase III, open-labelled, prospective RCT with $2 \times 2$ factorial design that compared survival advantage with the addition of mifamurtide to two chemotherapy treatments, regimen $A$ and regimen $B$. The two chemotherapy treatments were regimen A [cisplatin $\left(120 \mathrm{mg} / \mathrm{m}^{2}\right)$, doxorubicin $\left(75 \mathrm{mg} / \mathrm{m}^{2} /\right.$ day over 72 hours), and high-dose methotrexate (HDMTX) $\left.\left(12 \mathrm{mg} / \mathrm{m}^{2}\right)\right]$ and regimen B [(cisplatin $\left(120 \mathrm{mg} / \mathrm{m}^{2}\right)$, doxorubicin $(75$ $\mathrm{mg} / \mathrm{m}^{2} /$ day over 72 hours), high-dose methotrexate (HDMTX) $\left(12 \mathrm{mg} / \mathrm{m}^{2}\right)$ and ifosfamide $\left.\left(1.8 \mathrm{~g} / \mathrm{m}^{2}\right)\right]$, either with liposomal mifamurtide (regimen $A+/$ regimen $B+$ ), or without mifamurtide (regimen $A$ /regimen B-). Mifamurtide was administered at a dose of $2 \mathrm{mg} / \mathrm{m}^{2}$ intravenously during a one-hour period twice a week for 12 weeks, then once a week for 24 weeks. Patients enrolled in the study included those with newly diagnosed histologically confirmed high-grade intramedullary, nonmetastatic resectable osteosarcoma, and patients newly diagnosed with histologically confirmed high-grade intramedullary, metastatic osteosarcoma. At enrollment, patients were randomly assigned to one of the four treatment regimens, and all received 10 weeks of neoadjuvant chemotherapy as described above prior to definitive surgery during weeks 10-11. Later adjuvant chemotherapy was scheduled to begin at week 12 as per the randomly assigned treatment regimens. The survival outcomes for each patient population were analysed and reported as separate publications, at different time frames. One paper ${ }^{1}$ examined the event-free survival (EFS) and overall survival in metastatic osteosarcoma. The second paper ${ }^{2}$ examined EFS and overall survival in non-metastatic osteosarcoma patients. The preliminary results of these non-metastatic osteosarcoma patients were previously reported and published in 2005. ${ }^{31}$

Chou et al., 20091

Ninety-one patients (male=56 and female=35) were included in the study, with a median age of 13 years (range $=1-30$ years), who had osteosarcoma with at least one clinically detectable metastasis at enrollment. The comparison groups included 21 patients in regimen A- and 24 patients in regimen $B-$, while the groups receiving mifamurtide in addition to chemotherapy included 24 patients in regimen $\mathrm{B}+$, and 22 patients in regimen $\mathrm{A}+$. 
Meyers et al., $2008^{2}$

A total of 662 patients (male $=361$ and female $=301$ ) were included in the study, with a median age of 13 years (range $=1-30$ years), who had osteosarcoma without clinically detectable metastases. The comparison groups included 168 patients in regimen A- and 163 patients in regimen B-, while the groups receiving mifamurtide in addition to chemotherapy included 168 patients in regimen $\mathrm{B}+$, and 163 patients in regimen $A+$.

\section{Quasi-experimental before and after study}

The second study ${ }^{3}$ was a phase II, open-labelled, quasi-experimental study conducted from November 1988 to January 1992 at the MD Anderson Cancer Center (MDACC), Houston, USA. It examined PFS referred to as progression-free interval/disease-free interval in pulmonary metastatic/relapsed osteosarcoma patients. The study compared the time to progression with two different administration doses of liposomal mifamurtide in the experimental group (Group 1 and Group 2) to the chemotherapy treatment alone (Group 3 historical control group). Patients enrolled in the study had histologically confirmed osteosarcoma and pulmonary metastases, either present at the time of diagnosis, which persisted despite neoadjuvant chemotherapy, or that had developed during adjuvant chemotherapy or recurred following surgical excision. At study entry, these pulmonary metastatic and/or relapsed osteosarcoma patients received neoadjuvant chemotherapy treatment, following which their pulmonary tumours were surgically removed. All patients received postoperative chemotherapy. Patients in Group 3 received chemotherapy treatment which included a combination of one or more of the following agents: high-dose methotrexate with leucovorin rescue, cisplatin, etoposide, ifosfamide, cyclophosphamide and vincristine (administration doses for chemotherapy treatment were not reported). Group 1 patients received the same adjuvant chemotherapy along with mifamurtide at a dose of $2 \mathrm{mg} / \mathrm{m}^{2}$ infused over a one-hour period twice weekly for 12 weeks, whereas, patients in Group 2 received adjuvant chemotherapy along with the same dose of mifamurtide, twice weekly for 12 weeks and then once weekly for 12 weeks.

Data was collected from 49 patients ( 28 newly recruited patients and 21 historical patients). The age range was eight to 70 years, and there were 23 males and 26 females. Twelve patients were in Group 1, 16 patients were in Group 2, while Group 3 had 21 patients who had relapsed with lung metastasis following chemotherapy treatment and later underwent surgery to be free of visible tumour.

Refer to Appendix VII "Characteristics of included studies" for an overview of the included studies and findings.

\section{Methodological quality}

Papers underwent critical appraisal to assess risk of bias using the standardised JBI critical appraisal tool. Included papers were assessed against the ten critical appraisal questions applicable to RCTs and quasi-experimental studies. The results of the critical appraisal for each of the included papers are presented in Table 3. The critical appraisal instrument can be found in Appendix IV. The two papers describing the $\mathrm{RCT}^{1,2}$ provided a description for randomisation whereas the quasiexperimental study did not. ${ }^{3}$ The patients ${ }^{1,2}$ were assigned randomly to one of the four treatment 
regimens on enrollment by the registrar into a randomisation assignment sheet using a block size of four. The methods of allocation concealment to treatment allocation were not described clearly in any of the papers. Blinding of the treatment was difficult to implement in clinical practice for the following reasons: (1) the use of filters to reconstitute liposomes in the preparation of mifamurtide extended the treatment time, (2) the side effects that resulted post initial dose of mifamurtide, all of which had to be explained clearly to the patient and the family before administration, and (3) exposing 48 doses of mifamurtide over 36 weeks to children and adolescents was not acceptable due to mifamurtiderelated adverse events of which patients are informed at the beginning of the study, making it impossible to maintain blinding between the physician and patients. Furthermore, to assess patients' survival, blinding was not needed, the reason for which the study was open-label in nature. Blinding of outcome assessors was only described in the quasi-experimental study, while this was unclear in the other two papers describing the RCT.1,2 Both the treatment and control groups were comparable at study entry, with respect to the baseline information on patient characteristics such as age, sex, race and primary tumour site described in all three papers. The outcomes of patients who withdrew were described and reported in both RCTs.1,2 Reasons for withdrawal included discontinued adjuvant treatment due to disease progression, toxicity, and death due to a number of reasons which included infectious complications, operative complications and overdose of a self-administered illegal drug (which is a protocol deviation). Withdrawals were not explained or reported in the other study. ${ }^{3}$ Statistical analysis for the quasi-experimental study ${ }^{3}$ was described using survival in the SPSS/PC+ software (Statistical Package for the Social Sciences/PC+), which utilises Berkson-Gage survival tables and the Lee-Desu comparison test which is a generalisation of the Gehan-Breslow tests, while in the RCT,1,2 standard statistical methodologies were appropriately used for efficacy analysis and the outcomes were estimated by Kaplan-Meier method for each treatment regimen. The statistical significance of comparison of risk for analytical event/adverse event/death was then assessed by means of the log-rank test for the estimation of treatment differences with respect to EFS and overall survival. Confidence intervals (Cls) for relative risks were derived from the proportional hazards regression model. The three included papers were considered to be of reasonable methodological quality for inclusion in this review (with some limitations noted above), and there was no cut-off value for studies to be included.

Table 3: Results of critical appraisal of included randomised controlled trial/pseudorandomised trial

\begin{tabular}{|l|c|c|c|c|c|c|c|c|c|c|}
\hline Citation & Q1 & Q2 & Q3 & Q4 & Q5 & Q6 & Q7 & Q8 & Q9 & Q10 \\
\hline Chou et al., 20091 & Y & U & U & Y & U & Y & Y & Y & Y & Y \\
\hline Meyers et al., 20082 & Y & U & U & Y & U & Y & Y & Y & Y & Y \\
\hline Kleinerman et al., 19953 & N & U & U & N & Y & Y & Y & N & N & Y \\
\hline
\end{tabular}

$Q=$ Question; Y=Yes; N=No; U=Unclear;

Of the included studies, ${ }^{1-3}$ using the JBI classification system for ranking the level of evidence for effectiveness (Appendix I), two papers ${ }^{1,2}$ were classified as level 1.C and one paper ${ }^{3}$ was classified as level 2.d primary studies of effectiveness. 


\section{Narrative results}

The included studies were compared in terms of study population, interventions and outcome measures. Pooling of results was not possible due to a high degree of heterogeneity between study populations, interventions and outcome measures, therefore the findings are presented in a narrative form organised by outcome. The primary outcomes were event-free survival (EFS), overall survival, and recurrence of osteosarcoma. Secondary outcomes included health-related quality of life (HRQoL) and any mifamurtide-related adverse events as stated in the included papers. The outcomes were assessed at baseline, from the time of study commencement and after a period of adjuvant treatment with the mifamurtide.

\section{Primary outcomes}

\section{Event-free survival (EFS)}

The two papers based on one study reported EFS calculated in years, 1,2 whilst the second study described a different endpoint for treatment outcome with different terminology which was reported as progression-free survival (PFS) and/or time to progression calculated as a function of time measured in months. ${ }^{3}$ For the purpose of this review the authors decided it would be suitable to group the PFS outcome under EFS, as this outcome also measured prolonged survival time of osteosarcoma patients until any recurrence or tumour progression. The two papers detailing the RCT reported similar definitions for EFS.1,2 One paper defined it as "the time from study entry to progression of disease, diagnosis of second malignancy, death, or last follow-up whichever occurred first". 1(105341) The second paper defined it as "the time from study entry until adverse events or last patient contact, whichever came first. Adverse events included disease progression, diagnosis of second malignant neoplasm, or death before disease progression".2(10634) Here, the patients who had experienced disease progression, diagnosis of second malignancy or death were considered to have suffered an analytical event/adverse event. However, the second study ${ }^{3}$ defined PFS as "the time from the day of surgery to the day of relapse or disease progression".3(995) Meyers et al. ${ }^{2}$ reported EFS for the non-metastatic osteosarcoma patients ${ }^{2}$, while Chou et al. ${ }^{1}$ and Kleinerman et al. ${ }^{3}$ reported EFS and PFS for metastatic and relapsed osteosarcoma patients respectively.

In Chou et al. ${ }^{1}$ the five-year EFS in all the metastatic osteosarcoma patients who had received mifamurtide in addition to the chemotherapy regimen was $42 \%$ compared to $26 \%$ for patients who had received chemotherapy alone. The relative risk $[R R]$ for analytical events associated with randomisation to receive mifamurtide with the chemotherapy regimen was $0.72(95 \%$ confidence interval [CI], 0.42-1.2; $p=0.23$ ) (Table 4). The five-year EFS for each of the regimens were as follows: 1) Regimen $\mathrm{A}$ with mifamurtide $41 \%(95 \% \mathrm{Cl}, 0.21-0.60)$; 2) Regimen $\mathrm{A}$ without mifamurtide $29 \%$ $(95 \% \mathrm{Cl}, 0.11-0.51)$; 3) Regimen $\mathrm{B}$ with mifamurtide $44 \%(95 \% \mathrm{Cl}, 0.23-0.64)$; and 4) Regimen $\mathrm{B}$ without mifamurtide $23 \%(95 \% \mathrm{Cl}, 0.08-0.43)$. The proportional hazards regression $p$-value associated with the test of the hypotheses demonstrated no interaction between the chemotherapy intervention and mifamurtide intervention $(\mathrm{p}=0.20)$. This does not meet the conventional level of significance of less than 0.1 . The addition of mifamurtide to the chemotherapy regimen did not achieve a statistically significant improvement in EFS for the metastatic osteosarcoma patients. 
Table 4: Five-year EFS in metastatic osteosarcoma patients according to treatment regimen (Chou et al. ${ }^{1}$ )

\begin{tabular}{|l|c|c|c|c|}
\hline \multirow{2}{*}{ Treatment regimen } & $\begin{array}{c}\text { Probability of EFS } \\
(\%)\end{array}$ & $\begin{array}{c}\text { Relative risk of } \\
\text { adverse event }\end{array}$ & \multirow{2}{*}{$95 \% \mathrm{Cl}$} & \multirow{2}{*}{-value } \\
\cline { 2 - 3 } & 5 -year & & \\
\hline Regimen A- & $29 \%$ & & $0.11-0.51$ & \\
\hline Regimen A+ & $41 \%$ & & $0.21-0.60$ & \\
\hline Regimen B- & $23 \%$ & & $0.08-0.43$ & \\
\hline Regimen B+ & $44 \%$ & & $0.23-0.64$ & \\
\hline Regimen A-/Regimen B- & $26 \%$ & 1.0 & & \\
\hline Regimen A+/Regimen B+ & $42 \%$ & 0.72 & $0.42-1.2$ & $\mathrm{p}=0.23$ \\
\hline
\end{tabular}

EFS: Event-free survival

Cl: Confidence interval

Regimen A-: Patients treated with cisplatin, doxorubicin, and HDMTX (high dose methotrexate)

Regimen A+: Patients treated with cisplatin, doxorubicin, HDMTX, and mifamurtide

Regimen B-: Patients treated with cisplatin, doxorubicin, HDMTX, and ifosfamide

Regimen B+: Patients treated with cisplatin, doxorubicin, HDMTX, ifosfamide, and mifamurtide

The four-year and six-year EFS reported in Meyers et al..$^{2}$ for all the non-metastatic osteosarcoma patients randomised to receive mifamurtide in addition to chemotherapy was $69 \%$ and $67 \%$, respectively, whereas the four-year and six-year EFS for all non-metastatic osteosarcoma patients randomised to receive chemotherapy alone were $63 \%$ and $61 \%$, respectively (Table 5). The hazard ratio [HR] for patients who received mifamurtide was $0.80(95 \% \mathrm{Cl}, 0.62$ to $1.0 ; \mathrm{p}=.08)$. The four-year and six-year EFS for each of the regimens were as follows: 1) Regimen A with mifamurtide $65 \%$ and $63 \%$, respectively; 2) Regimen A without mifamurtide $66 \%$ and $64 \%$, respectively; 3 ) Regimen B with mifamurtide $74 \%$ and $71 \%$, respectively; and 4) Regimen B without mifamurtide $60 \%$ and $58 \%$, respectively. The proportional hazards regression $p$-value associated with the test of the hypotheses demonstrated no interaction between the chemotherapy intervention and mifamurtide intervention $(p=.102)$. This does not meet the conventional level of significance of less than 0.1 . There was a trend towards improvement in EFS for non-metastatic osteosarcoma patients who had received mifamurtide in addition to the chemotherapy regimen however it did not achieve a statistically significant difference. 
Table 5: Four-year and six-year EFS in non-metastatic osteosarcoma patients according to treatment regimen (Meyers et al. ${ }^{2}$ )

\begin{tabular}{|l|c|c|c|c|c|}
\hline \multirow{2}{*}{ Treatment regimen } & \multicolumn{2}{|c|}{$\begin{array}{c}\text { Probability of EFS } \\
(\%)\end{array}$} & $\begin{array}{c}\text { Hazard } \\
\text { ratio of } \\
\text { EFS }\end{array}$ & \multirow{2}{*}{$\mathbf{9 5 \%} \mathrm{Cl}$} & p-value \\
\cline { 2 - 3 } & 4 -year & 6 -year & EF & \\
\hline Regimen A- & $66 \%$ & $64 \%$ & & & \\
\hline Regimen A+ & $65 \%$ & $63 \%$ & & & \\
\hline Regimen B- & $60 \%$ & $58 \%$ & & & \\
\hline Regimen B+ & $74 \%$ & $71 \%$ & & & \\
\hline Regimen A-/Regimen B- & $63 \%$ & $61 \%$ & & & \\
\hline Regimen A+/Regimen B+ & $69 \%$ & $67 \%$ & 0.80 & $0.62-1.0$ & $\mathrm{p}=0.08$ \\
\hline
\end{tabular}

EFS: Event-free survival

$\mathrm{Cl}$ : Confidence interval

Regimen A-: Patients treated with cisplatin, doxorubicin, and HDMTX (high dose methotrexate)

Regimen A+: Patients treated with cisplatin, doxorubicin, HDMTX, and mifamurtide

Regimen B-: Patients treated with cisplatin, doxorubicin, HDMTX, and ifosfamide

Regimen B+: Patients treated with cisplatin, doxorubicin, HDMTX, ifosfamide, and mifamurtide

In Kleinerman et al., ${ }^{3}$ the median time to progression for pulmonary metastatic osteosarcoma patients in Group 1 was 6.8 months and in Group 2 was nine months, when compared to 4.5 months for the control Group 3 (Table 6). Hence, the addition of mifamurtide to the adjuvant chemotherapy regimen in Group 1 did not show a statistically significant difference in PFS compared to patients in control Group 3, with $p=.18$, however, there was a statistically significant difference in PFS in Group 2 compared to patients in control Group 3, with $p<.03$, (Table 6).

Table 6: PFS in pulmonary metastatic osteosarcoma patients according to treatment regimen (Kleinerman et al. ${ }^{3}$ )

\begin{tabular}{|l|l|l|}
\hline Treatment regimen (n) & $\begin{array}{l}\text { Median time to } \\
\text { progression }\end{array}$ & p-value \\
\hline${ }^{1}$ Group 1 (12) & 6.8 months & \\
\hline${ }^{2}$ Group 2 (16) & 9 months & \\
\hline${ }^{3}$ Group 3 (21) & 4.5 months & \\
\hline Group1 vs Group 3 & & $p=0.18$ \\
\hline Group 2 vs Group 3 & & $p<0.03$ \\
\hline
\end{tabular}

PFS: Progression-free survival

${ }^{1}$ All patients in this group received mifamurtide twice weekly for 12 weeks along with adjuvant chemotherapy

${ }^{2}$ All patients in this group received mifamurtide twice weekly for 12 weeks and then once weekly for 12 weeks along with adjuvant chemotherapy

${ }^{3}$ All patients in this historical control group had received adjuvant chemotherapy alone consisting of one or more of the following agents: high-dose methotrexate with leucovorin, cisplatin, etoposide, ifosfamide, cyclophosphamide, and vincristine 


\section{Overall survival}

Two papers from the same study reported overall survival in years, defined as "the time from study entry to death or last follow-up, whichever occurred first" 1(p5341) and "the time from entry until death or last patient contact". 2(p634) The third paper described a different endpoint for treatment outcome with different terminology reported as survival after relapse in months. ${ }^{3}$ This paper ${ }^{3}$ lacked a clear definition of overall survival but stated that the length of survival following surgery was analysed by life-table analysis. For the purpose of this review, the authors decided it would be suitable to group the survival after relapse outcome under overall survival, as this outcome was also measured by prolonged survival time of osteosarcoma patients until death or last follow-up. Meyers et al. ${ }^{2}$ reported overall survival for the non-metastatic osteosarcoma patients, while Chou et al. ${ }^{1}$ and Kleinerman et al. ${ }^{3}$ reported overall survival and survival after relapse for metastatic and relapsed osteosarcoma patients, respectively.

The five-year overall survival ${ }^{1}$ in the metastatic osteosarcoma patients randomised to receive mifamurtide in addition to the chemotherapy regimen was $53 \%$ compared to $40 \%$ for patients who received the chemotherapy regimen alone. The RR for death for patients randomised to receive mifamurtide was $0.72(95 \% \mathrm{Cl}, 0.40-1.3 ; p=0.27)$ (Table 7). The five-year overall survival for each of the regimens was as follows: 1) Regimen A with mifamurtide 50\% (95\% Cl, 26\%-69\%); 2) Regimen A without mifamurtide $53 \%$ (95\% Cl, 28\%-73\%); 3) Regimen B with mifamurtide $57 \%$ (95\% Cl, 33\%$75 \%)$; 4) Regimen B without mifamurtide $30 \%$ (95\% Cl, 13\%-50\%) (Table 7). There was no statistically significant difference among the treatment regimens $(p=0.60$, long-rank test). The proportional hazards regression $p$-value associated with the test of the hypotheses had no interaction between the chemotherapy intervention and mifamurtide intervention $(p=0.39)$. This does not meet the conventional level of significance of less than 0.1 . Hence, the addition of mifamurtide to the chemotherapy regimen did not achieve a statistically significant improvement in overall survival for the metastatic osteosarcoma patients. 
Table 7: Five-year overall survival in metastatic osteosarcoma patients according to treatment regimen (Chou et al. ${ }^{1}$ )

\begin{tabular}{|l|c|c|c|c|}
\hline \multirow{2}{*}{ Treatment regimen } & $\begin{array}{c}\text { Overall survival } \\
\text { Probability (\%) }\end{array}$ & $\begin{array}{c}\text { Relative } \\
\text { risk of } \\
\text { death }\end{array}$ & $\mathbf{9 5 \% ~ C l}$ & p-value \\
\cline { 2 - 5 } & 5 -year & & $0.28-0.73$ & \\
\hline Regimen A- & $53 \%$ & & $0.26-0.69$ & \\
\hline Regimen A+ & $50 \%$ & & $0.13-0.50$ & \\
\hline Regimen B- & $30 \%$ & & $0.33-0.75$ & \\
\hline Regimen B+ & $57 \%$ & & & \\
\hline Regimen A-/Regimen B- & $40 \%$ & & $0.40-1.3$ & $\mathrm{p}=0.27$ \\
\hline Regimen A+/Regimen B+ & $53 \%$ & 0.72 & & \\
\hline
\end{tabular}

Cl: Confidence interval

Regimen A-: Patients treated with cisplatin, doxorubicin, and HDMTX (high dose methotrexate)

Regimen A+: Patients treated with cisplatin, doxorubicin, HDMTX, and mifamurtide

Regimen B-: Patients treated with cisplatin, doxorubicin, HDMTX, and ifosfamide

Regimen B+: Patients treated with cisplatin, doxorubicin, HDMTX, ifosfamide, and mifamurtide

The four-year and six-year overall survival 2 in non-metastatic osteosarcoma patients randomised to receive mifamurtide in addition to the chemotherapy regimen was $84 \%$ and $78 \%$ respectively. While, the four-year and six-year overall survival in non-metastatic osteosarcoma patients who received chemotherapy alone was $78 \%$ and $70 \%$, respectively (Table 8 ). The RR of death for patients randomised to receive mifamurtide was $0.71(95 \% \mathrm{Cl}, 0.52$ to $0.96 ; p=0.03)$. The four-year and sixyear overall survival for each of the regimens was as follows: 1) Regimen $A$ with mifamurtide $82 \%$ and $75 \%$, respectively; 2) Regimen A without mifamurtide $78 \%$ and $71 \%$ respectively; 3 ) Regimen B with mifamurtide $86 \%$ and $81 \%$, respectively; 4) Regimen B without mifamurtide $77 \%$ and $70 \%$, respectively. The proportional hazards regression $p$-value associated with the test of the hypotheses had no interaction between the chemotherapy intervention and mifamurtide intervention and was $p=0.60$. This level of significance does not meet the standard of level of less than 0.1 . The addition of mifamurtide to the chemotherapy regimen achieved a statistically significant improvement in overall survival in non-metastatic osteosarcoma patients. 
Table 8: Four-year and six-year overall survival in non-metastatic osteosarcoma patients according to treatment regimen (Meyers et al. ${ }^{2}$ )

\begin{tabular}{|l|c|c|l|l|l|}
\hline \multirow{2}{*}{ Treatment regimen } & \multicolumn{2}{|c|}{$\begin{array}{l}\text { Overall survival } \\
\text { Probability (\%) }\end{array}$} & $\begin{array}{l}\text { Relative } \\
\text { risk of } \\
\text { death }\end{array}$ & $95 \% \mathrm{Cl}$ & p-value \\
\cline { 2 - 6 } & 4-year & $\mathbf{6 - y e a r}$ & & \\
\hline Regimen A- & $78 \%$ & $71 \%$ & & & \\
\hline Regimen A+ & $82 \%$ & $75 \%$ & & & \\
\hline Regimen B- & $77 \%$ & $70 \%$ & & & \\
\hline Regimen B+ & $86 \%$ & $81 \%$ & & & \\
\hline Regimen A-/Regimen B- & $78 \%$ & $70 \%$ & & & \\
\hline Regimen A+/Regimen B+ & $84 \%$ & $78 \%$ & 0.71 & $0.52-0.96$ & $\mathrm{p}=0.03$ \\
\hline
\end{tabular}

$\mathrm{Cl}$ : Confidence interval

Regimen A-: Patients treated with cisplatin, doxorubicin, and HDMTX (high dose methotrexate)

Regimen A+: Patients treated with cisplatin, doxorubicin, HDMTX, and mifamurtide

Regimen B-: Patients treated with cisplatin, doxorubicin, HDMTX, and ifosfamide

Regimen B+: Patients treated with cisplatin, doxorubicin, HDMTX, ifosfamide, and mifamurtide

Kleinerman et al. ${ }^{3}$ reported survival after relapse in pulmonary metastatic and/or relapsed osteosarcoma patients. The median survival time for pulmonary metastatic and/or relapsed osteosarcoma patients in Group 1 was 40.5 months and Group 2 was $>44.0$ months when compared to 10.5 months for the control Group 3 (Table 9). Hence, the addition of mifamurtide to the adjuvant chemotherapy regimen in both Group 1 and Group 2 did show statistically significant differences in survival after relapse compared to patients in the control Group 3, with $p<0.01$ and $p<0.04$, respectively. However, the significance of the data for Group 2 is interpreted here with caution, as this group entered the study later, and thus had a shorter follow-up time.

Table 9: Survival after relapse in pulmonary metastatic and/or relapsed osteosarcoma patients according to treatment regimen (Kleinerman et al. ${ }^{3}$ )

\begin{tabular}{|l|l|l|}
\hline Treatment regimen (n) & Median survival time & p-value \\
\hline${ }^{1}$ Group 1 (12) & 40.5 months & \\
\hline${ }^{2}$ Group 2 (16) & $>44.0$ months & \\
\hline${ }^{3}$ Group 3 (21) & 10.5 months & \\
\hline Group1 vs Group 3 & & $p<0.01$ \\
\hline Group2 vs Group 3 & & $p<0.04$ \\
\hline
\end{tabular}

\footnotetext{
${ }^{1}$ All patients in this group received mifamurtide twice weekly for 12 weeks along with adjuvant chemotherapy

${ }^{2}$ All patients in this group received mifamurtide twice weekly for 12 weeks and then once weekly for 12 weeks along with adjuvant chemotherapy

${ }^{3}$ All patients in this historical control group had received adjuvant chemotherapy alone consisting of one or more of the following agents: high-dose methotrexate with leucovorin, cisplatin, etoposide, ifosfamide, cyclophosphamide, and vincristine
} 


\section{Recurrence of osteosarcoma}

Meyers et al. ${ }^{2}$ and Kleinerman et al. ${ }^{3}$ reported recurrence of osteosarcoma. In Meyers et al., ${ }^{2}$ $217 / 662$ (33\%) patients reported recurrence of osteosarcoma measured by chest X-ray undertaken monthly, lung CT scan repeated every three months, and/or bone scans every six months. However, the data was not available for individual groups, whereas in Kleinerman et al., ${ }^{3}$ eight out of 12 patients in Group 1 had recurrence of osteosarcoma in the area of the surgery of primary tumour that had been surgically removed before the study entry which was assessed and demonstrated by chest X-ray monthly, lung CT scan repeated every three months, bone scans every six months and metastasis pathological examination. The metastasis pathological examination was undertaken for any histological changes of pulmonary tumour or reappearance of malignant characteristics of primary tumour. However, data for other groups, Group 2 and Group 3 was not available.

\section{Secondary outcomes}

\section{Any mifamurtide-related adverse events}

Chou et al. ${ }^{1}$ and Kleinerman et al. ${ }^{3}$ reported mifamurtide-related adverse events. In Chou et al. ${ }^{1}$ mifamurtide-related adverse events were reported as Grade 3 (severe) and Grade 4 (lifethreatening) toxicity, for which grading was based on the Children's Cancer Group Toxicity and Complication Criteria (a scale partially similar to the National Cancer Institute's Common Toxicity Criteria). Grade 3 and Grade 4 toxicity included haematological toxicity (white blood cell count, absolute neutrophil count, platelets, haemoglobin); hepatic (aspartate aminotransferase, alanine aminotransferase, alkaline phosphatase, total bilirubin); renal (creatinine, creatinine clearance); gastrointestinal disorders (stomatitis, nausea and vomiting); cardiac rhythm; nervous system disorder (peripheral sensory, centrocerebellar; skin disorders); ear disorders (hearing-objective); and others (infection, fever, performance status) (Table 10). In this study, no deaths were reported in association with mifamurtide-related adverse events. The number of mifamurtide-related adverse events appeared to be similar across all of the combined treatment regimens. No statistical analysis was undertaken, however, the figures suggest there were no significant differences between the treatment regimens (Table 10). Kleinerman et al. ${ }^{3}$ reported mifamurtide-related adverse events as clinical toxic effects of mifamurtide. All patients in both Group 1 and Group 2, who had received the initial single dose of mifamurtide reported having chills which began 15 minutes to 1.5 hours postinfusion and lasted up to one hour, fever (one to three hours postinfusion and occurred within two hours of chills), and headache (one to two hours postinfusion). For the subsequent doses of mifamurtide in Group 1 and Group 2, all patients reported Grade 1 toxicity as delayed fatigue. 
Table 10: Mifamurtide-related adverse events in metastatic osteosarcoma patients according to treatment regimen (Chou et al. 2009')

\begin{tabular}{|c|c|c|c|c|c|c|c|c|c|}
\hline \multirow[t]{2}{*}{ Toxicity } & \multicolumn{2}{|c|}{$\begin{array}{l}\text { Regimen A- } \\
(n=21)\end{array}$} & \multicolumn{2}{|c|}{$\begin{array}{l}\text { Regimen } \mathrm{A}^{+} \\
(\mathrm{n}=22)\end{array}$} & \multicolumn{2}{|c|}{$\begin{array}{l}\text { Regimen B- } \\
(n=24)\end{array}$} & \multicolumn{2}{|c|}{$\begin{array}{l}\text { Regimen } B+ \\
(n=24)\end{array}$} & \multirow{2}{*}{ 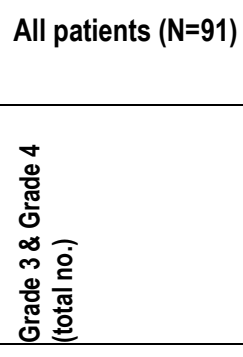 } \\
\hline & 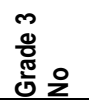 & 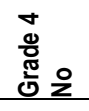 & 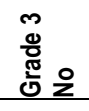 & 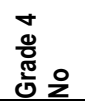 & 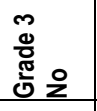 & 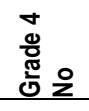 & 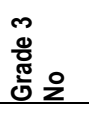 & 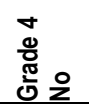 & \\
\hline \multicolumn{10}{|l|}{ Hematologic toxicity } \\
\hline White blood cell count & 3 & 3 & 2 & 1 & & 6 & 2 & 6 & 23 \\
\hline Absolute neutrophil count & 1 & 9 & 3 & 5 & & 6 & 3 & 8 & 35 \\
\hline Platelets & 2 & 4 & 1 & 3 & 1 & 2 & 2 & 6 & 21 \\
\hline Haemoglobin & 2 & 1 & & 1 & 1 & & 1 & 3 & 9 \\
\hline \multicolumn{10}{|l|}{$\underline{\text { Hepatic }}$} \\
\hline Aspartate aminotransferase & 7 & 2 & 6 & 3 & 7 & 3 & 10 & 3 & 41 \\
\hline Alanine aminotransferase & 6 & 5 & 6 & 4 & 12 & 4 & 11 & 6 & 54 \\
\hline Alkaline phosphatase & 1 & & & & & & & 1 & 2 \\
\hline total bill & 2 & 1 & & & & & 2 & 2 & 7 \\
\hline \multicolumn{10}{|l|}{$\underline{\text { Renal }}$} \\
\hline Creatinine & & & & & & & 1 & & 1 \\
\hline Creatinine clearance & & & & 1 & 1 & & & 2 & 4 \\
\hline \multicolumn{10}{|l|}{ Gastrointestinal disorders } \\
\hline Stomatitis & 2 & 5 & 4 & 3 & 5 & 2 & 2 & 7 & 30 \\
\hline Nausea and vomiting & 1 & 3 & 4 & 1 & 2 & & 4 & 1 & 16 \\
\hline Cardiac rhythm & & & & & 1 & & & 1 & 2 \\
\hline \multicolumn{10}{|l|}{$\underline{\text { Nervous system disorders }}$} \\
\hline Peripheral sensory & 1 & & & & & & & & 1 \\
\hline Centrocerebellar & 1 & & & & & & 1 & 1 & 3 \\
\hline Skin disorders & & & & & 1 & & 1 & & 2 \\
\hline Hearing-objective & & & 1 & & 2 & & 1 & & 4 \\
\hline Infection & 4 & & 3 & 1 & 5 & & 4 & 1 & 18 \\
\hline Fever & & & 1 & & & & 2 & 1 & 4 \\
\hline Performance status & & 1 & & & & & & & 1 \\
\hline
\end{tabular}

Regimen A-: Patients treated with cisplatin, doxorubicin, and HDMTX (high dose methotrexate)

Regimen A+: Patients treated with cisplatin, doxorubicin, HDMTX, and mifamurtide

Regimen B-: Patients treated with cisplatin, doxorubicin, HDMTX, and ifosfamide

Regimen B+: Patients treated with cisplatin, doxorubicin, HDMTX, ifosfamide, and mifamurtide 


\section{Health-related quality of life (HRQoL)}

None of the included studies measured this outcome.

Chapter 5 discusses the findings and limitations of the systematic review. This chapter also outlines the conclusion and ends with the implications for practice and research. 


\section{CHAPTER 5: DISCUSSION AND CONCLUSION}

The final chapter of this thesis has three objectives. The first is to provide an overview of findings of the review, highlighting the effectiveness of mifamurtide in addition to standard chemotherapy on primary and secondary outcomes for high-grade osteosarcoma. The second objective is to discuss the limitations of the review. Lastly the chapter concludes with remarks regarding implications of this review for practice and research.

\section{Overview of findings}

The aim of this systematic review was to identify the best available evidence on the effectiveness of mifamurtide in addition to standard chemotherapy in the treatment of high-grade, resectable, nonmetastatic and metastatic osteosarcoma. This systematic review included two studies, based on three papers (a total of 802 patients), with the age range of one to 70 years. None of the included studies mentioned or performed any subgroup analysis by age. Of the included studies, two papers ${ }^{1,2}$ were classified as level 1.c and the third paper $^{3}$ as level 2.d primary studies of effectiveness, based on the JBI classification system for ranking Levels of Evidence (Appendix I). The review compared groups receiving mifamurtide in addition to chemotherapy to groups receiving chemotherapy alone, and measured EFS, overall survival, recurrence of osteosarcoma and mifamurtide-related adverse events. The included studies provided a relatively sparse amount of data, and due to heterogeneity of populations and interventions, and available studies, meta-analysis could not be undertaken.

\section{Effectiveness of mifamurtide in addition to standard chemotherapy on EFS for high-grade osteosarcoma}

Two papers ${ }^{1,2}$ identified that, for EFS, the groups receiving mifamurtide in addition to adjuvant chemotherapy regimen demonstrated a non-significant statistical improvement in non-metastatic osteosarcoma patients and no statistically significant improvement in metastatic osteosarcoma patients. However, the third paper ${ }^{3}$ identified that, for progression-free survival (PFS), one out of the two treatment groups showed a statistically significant difference in pulmonary metastatic and/or relapsed osteosarcoma patients, favouring the addition of mifamurtide to the chemotherapy regimen.

\section{Effectiveness of mifamurtide in addition to standard chemotherapy on overall survival for high-grade osteosarcoma}

The two included papers ${ }^{1,2}$ from the same study identified that, for overall survival, the groups receiving mifamurtide in addition to the adjuvant chemotherapy regimen resulted in a statistically significant improvement in non-metastatic osteosarcoma patients ${ }^{2}$, while no statistically significant improvement was evident in metastatic osteosarcoma patients. ${ }^{1}$ It should be noted that for metastatic osteosarcoma patients, a small number of patients were enrolled in the study, ${ }^{1}$ which possibly decreased the power to detect statistically significant differences between treatment regimens. The third paper ${ }^{3}$ identified that, for survival after relapse, both the treatment groups 
showed a statistically significant difference in pulmonary metastatic and/or relapsed osteosarcoma patients, favouring the addition of mifamurtide to the chemotherapy regimen.

\section{Recurrence of osteosarcoma}

This review did reveal recurrence of osteosarcoma in non-metastatic ${ }^{2}$ and relapsed ${ }^{3}$ osteosarcoma patients. For non-metastatic patients, ${ }^{2}$ recurrence of osteosarcoma was reported, inclusive of all the four treatment groups. For relapsed osteosarcoma patients, ${ }^{3}$ recurrence was only seen in group 1 in the area of surgery of the primary tumour that had been surgically removed before study entry.

\section{Effectiveness of mifamurtide in addition to standard chemotherapy on mifamurtide-related adverse events and HRQoL for high-grade osteosarcoma}

One study ${ }^{1}$ identified that, for metastatic osteosarcoma patients, mifamurtide-related adverse events were demonstrated as Grade 3 and/or Grade 4 toxicity for which no statistical analysis was undertaken. Grade 3 and/or Grade 4 toxicity included haematological, hepatic, renal, gastrointestinal disorders, cardiac rhythm, nervous system disorders, ear disorders and others (infection, fever and performance status) in metastatic osteosarcoma patients. However, the findings of this review show that Grade 3 and/or Grade 4 toxicity appeared to be similar across all of the combined treatment regimens groups. However, the other study 3 identified that, for the relapsed osteosarcoma patients, mifamurtide-related adverse events were demonstrated as clinical toxic effects of mifamurtide, which included post initial dose (chills, fever, headache) and post subsequent doses (Grade 1 toxicity as delayed fatigue). The other secondary outcome, HRQLL, was not measured.

\section{Limitations of the review}

The first limitation of this review relates to its scope; only studies published in the English language were sought and considered for inclusion. There may be additional non-English language studies (one such study (a Polish paper ${ }^{78}$ ) was identified but excluded from this review) that may have contributed useful data which may have led to different conclusions being drawn. The methodological weaknesses in the design of included studies relating to performance, attrition and detection bias, (as summarised in Table 3) are a second limitation. A third limitation is that three other potential papers were excluded since there was no access to full text (only published conference proceedings and meeting abstracts were available), making quality assessment impossible. A fourth limitation is the inability to pool the results to conduct a meta-analysis due to the high degree of heterogeneity between study populations, interventions and outcome measures. For example, patients in the three included papers may have had resectable or nonresectable disease and non-metastatic, metastatic or relapsed osteosarcoma. Lastly, differences in terminology and definitions of some outcomes (EFS and overall survival) were used in the included studies. The authors were contacted for clarification where required, however, no responses were received. The use of universal terminology needs to be implemented to assist future clinical practice and research.

Given that the above were the only outcome measures to determine the effectiveness of mifamurtide in addition to standard chemotherapy regimen, and that this is the only known systematic review on this topic, it was impossible to align this review with the international literature. There were some 
lower level descriptive studies identified $40,50,59,60$ during the search process that were subsequently not included in this review as the preference was on experimental research. Like the included studies, some of the descriptive studies varied in terms of the terminologies used and the definitions of some of the outcomes reported. These descriptive studies measured PFS, overall survival, recurrence of osteosarcoma and mifamurtide-related adverse events as clinical toxic side effects in metastatic and/or metastatic recurrent osteosarcoma patients. Results of these studies were similar to the results of the included studies in this review. One descriptive study59 included pulmonary metastatic and/or relapsed osteosarcoma patients. One group was treated with liposomal mifamurtide along with chemotherapy - ifosfamide, followed by the surgical resection of metastases. The other group underwent surgical resection of metastases first, followed by liposomal mifamurtide with chemotherapy - ifosfamide). In terms of PFS, it showed similar median time of disease progression to one of the included papers ${ }^{3}$ of this review, however, no statistical analysis was undertaken. In another descriptive study by Anderson et al., ${ }^{60}$ the two-year overall survival ${ }^{60}$ was $45.9 \%$ for the entire cohort receiving liposomal mifamurtide along with chemotherapy in metastatic and/or relapsed osteosarcoma patients, which was similar to one of the included papers ${ }^{1}$ of this review, which reported five-year overall survival in metastatic osteosarcoma patients. The third descriptive study ${ }^{40}$ reported recurrence of osteosarcoma in two out of nine patients at the third month and the $12^{\text {th }}$ month, respectively, in specimens obtained after liposomal mifamurtide treatment measured by X-ray, lung CT and metastasis pathological examination. It was very similar to the quasi-experimental before and after study 3 included in the review because the tumour recurred at a similar location, in the area of surgery that had been performed immediately before liposomal mifamurtide treatment which were excised to be compared with the specimens before the treatment. The descriptive studies that described mifamurtide-related adverse events as clinical toxic effects $40,50,59,60$ were similar to that reported in the included paper ${ }^{3}$ of this review, which included Grade 240,50,59,60 (headache) and Grade 140,50,59,60 (delayed fatigue) effects. However, another descriptive study 60 reported Grade $\geq 3^{60}$ (pyrexia) such as pericarditis or pleural effusions. In this study 60 Grade 1 or Grade 2 effects were reported as mifamurtide-associated infusion-related adverse events (IRAEs) in patient self-reporting diaries, and no statistical analysis was undertaken. The interpretation from immune response and histological examination did provide some evidence that inclusion of mifamurtide in chemotherapy may have some beneficial effects ${ }^{40,50,59,60}$ on metastatic osteosarcoma patients, which may be capable enough to eradicate residual micrometastases that are not eliminated by chemotherapy alone. It is, however, unclear whether these results could be correlated with tumour response, as altogether the numbers of patients were too small to determine the beneficial effects, although mifamurtide was safe and tolerated in the majority of patients. In support of these descriptive studies, larger groups of patients would be needed in future research.

Treatment with mifamurtide is an additional cost, therefore funding is required to enable this drug to be available commercially for routine use in the frontline therapy of newly diagnosed high-grade osteosarcoma patients. Mifamurtide has been approved for use by the European Medicines Agency (EMA) for the treatment of non-metastatic, resectable osteosarcoma since March 2009, specifically in pediatric patients, ${ }^{79,80}$ following which, in 2010 , mifamurtide was also available for clinical use in Germany and Austria. Additionally, mifamurtide has also been included in the treatment of 
osteosarcoma in many countries including the European Union, Central and South America, Israel and Turkey. Mifamurtide may also be available in other countries, through a "Named Patient Program" (NPP), a specific type of expanded access where a physician can access a noncommercialised product for a particular "named" patient through a regulatory authorisation process. However, in the US, mifamurtide still remains an investigational agent as the Food and Drug Administration (FDA) has refused the marketing approval of mifamurtide due to insufficient evidence of a survival advantage. In Australia, mifamurtide is classified under prescription medicines as an orphan drug by the Therapeutic Goods Administration (TGA) Australia. ${ }^{81}$ An orphan drug ${ }^{81}$ is defined as "a medicine, vaccine or in vivo diagnostic agent, if it complies with this regulation. It: (a) must be intended to treat, prevent or diagnose a rare disease; or (b) must not be commercially viable to supply to treat, prevent or diagnose another disease or condition". ${ }^{2(p 51)}$ However, orphan drugs are only eligible for a waiver through an application form (fees exempted) that needs to be approved by the secretary or the delegate secretary of the TGA, Department of Health, in order to register it on the Australian Register of Therapeutic Goods (ARTG). ${ }^{82}$ An orphan drug is still considered as a higher risk medicine, although registered on the ARTG and approved by the TGA, following the evaluation for its quality, safety and efficacy.

\section{Conclusion}

The evidence on the effectiveness of mifamurtide in addition to the chemotherapy regimen to treat clinically detectable high-grade, resectable, non-metastatic, metastatic, and/or relapsed osteosarcoma in the hospital setting is sparse. There is little evidence to suggest that treatment with mifamurtide can either improve or reduce EFS, overall survival or recurrence of osteosarcoma. Some mifamurtide-related adverse events were noted such as haematological toxicity, hepatic, renal, gastrointestinal disorders, cardiac rhythm, nervous system disorders, ear disorders and others (infection, fever and performance status). There is currently no data available on the effect of mifamurtide on HRQoL. Furthermore, due to the small number of studies on mifamurtide as an adjunct treatment, and the quality of the currently available studies, it is impossible to confirm or refute whether mifamurtide in addition to a chemotherapy regimen is better than the gold standard chemotherapy regimen alone. More high-quality RCTs are needed to provide reliable evidence in order to evaluate the effectiveness of mifamurtide in addition to the standard chemotherapy regimens, despite osteosarcoma being a rare type of primary bone cancer with a high rate of tumour heterogeneity. However, this review does suggest that mifamurtide may deserve further investigation to define its role in the treatment of high-grade osteosarcoma as an adjunct to chemotherapy treatment that might further improve the survival outcomes in patients affected by this rare disease. Fortunately, the current existing global collaborative networks that support clinical trials for osteosarcoma will be a key asset in addressing further opportunities in the future.

\section{Implications for practice}

The results of this systematic review suggest that currently there is no merit for the inclusion of mifamurtide as an adjuvant therapy in addition to standard chemotherapy regimen in the treatment of high-grade, resectable, non-metastatic, metastatic, and/or recurrent osteosarcoma in the hospital setting. According to the new JBI Grades of Recommendation (Appendix II), this can be graded as 
grade $B$. This review identified no evidence to suggest that mifamurtide in addition to a chemotherapy regimen was actually beneficial to osteosarcoma patients. Additionally, mifamurtiderelated adverse events were identified. However, until further evidence to either support or refute the use of mifamurtide as an adjuvant therapy is uncovered, practice should be guided by prescriber knowledge, involving patient preference and local policy. A 'Summary of Findings' table was created using GRADE approach is presented in Table 11. 
Table 11: Summary of Findings

\section{Mifamurtide in addition to standard chemotherapy compared to chemotherapy alone for patients with high- grade non-metastatic and metastatic osteosarcoma}

Patient or population: patients with high-grade non-metastatic and metastatic osteosarcoma

Setting: Outpatient University hospital and rehabilitation setting

Intervention: mifamurtide in addition to standard chemotherapy

Comparison: chemotherapy alone

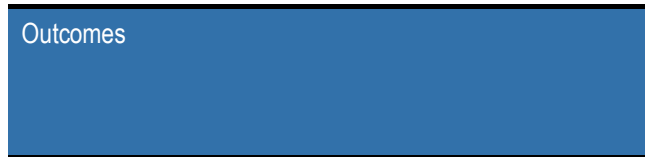

Event-free survival and Progression-free survival (EFS and PFS ) assessed with: in years and months respectively

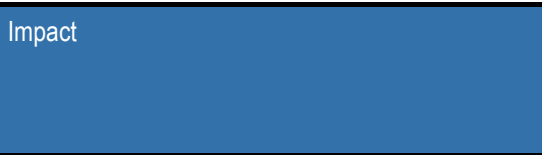

In Chou et al., 1 for 5 year EFS, the relative risk [RR] 802 for analytical events associated with randomisation to receive mifamurtide with the chemotherapy regimen was $0.72(95 \% \mathrm{Cl}, 0.42-1.2 ; \mathrm{p}=0.23)$ in metastatic osteosarcoma patients. In Meyers et al., ${ }^{2}$ for 4 year and 6 year EFS, the Hazard ratio [HR] for patients who received mifamurtide was $0.80(95 \% \mathrm{Cl}$, 0.62 to $1.0 ; p=0.08$ ) in non-metastatic osteosarcoma patients. In Kleinerman et al., ${ }^{3}$ for PFS one out of the two treatment groups showed a statistically significant difference, with $p<0.03$ in pulmonary metastatic and/or relapsed osteosarcoma patients, favoring the addition of mifamurtide to the chemotherapy regimen.

\begin{tabular}{|l|l|}
\hline $\begin{array}{l}\text { № of } \\
\text { participants } \\
\text { (studies) }\end{array}$ & $\begin{array}{l}\text { Quality of } \\
\text { the evidence } \\
\text { (GRADE) }\end{array}$ \\
\hline
\end{tabular}

(2 RCTs)

$\bigoplus \bigoplus \bigoplus \bigcirc$ MODERATE
Overall survival and survival after relapse assessed with: in years and months respectively

\begin{abstract}
In Chou et al., ${ }^{1}$ for 5 year overall survival, the RR for 802 death for patients randomised to receive mifamurtide was $0.72(95 \% \mathrm{Cl}, 0.40-1.3 ; p=0.27)$ in metastatic osteosarcoma patients. In Meyers et al., ${ }^{2}$ for 4 year and 6 year overall survival, the RR of death for patients randomised to receive mifamurtide was $0.71(95 \% \mathrm{Cl}, 0.52$ to $0.96 ; p=0.03)$ in non-metastatic osteosarcoma patients. In Kleinerman et al., ${ }^{3}$ for survival after relapse both the treatment groups, showed a statistically significant difference, with $p<0.01$ and $p<0.04$ in pulmonary metastatic and/ or relapsed osteosarcoma patients respectively, favoring the addition of mifamurtide to the chemotherapy regimen.
\end{abstract}

Recurrence of osteosarcoma assessed with: chest Xray undertaken monthly, lung $C T$ scan repeated every 3 months, and/or bone scans every 6 months
In Chou et al., ${ }^{1}$ and Kleinerman et al. ${ }^{3}$ recurrence of 140 osteosarcoma were revealed in non-metastatic (2RCTs) [217/662 (33\%)] and relapsed osteosarcoma patients $(8 / 12$, Group 1$)$ respectively.

$\begin{array}{ll}802 & \bigoplus \bigoplus \bigoplus \bigcirc \\ (2 \mathrm{RCTS}) & \substack{\text { MODERATE } \\ \text { a }}\end{array}$

$\oplus \oplus \oplus \bigcirc$ MODERATE a

${ }^{*}$ The risk in the intervention group (and its $95 \%$ confidence interval) is based on the assumed risk in the comparison group and the relative effect of the intervention (and its $95 \% \mathrm{Cl}$ ).

Cl: Confidence interval

GRADE Working Group grades of evidence

High quality: We are very confident that the true effect lies close to that of the estimate of the effect

Moderate quality: We are moderately confident in the effect estimate: The true effect is likely to be close to the estimate of the effect, but there is a possibility that it is substantially different

Low quality: Our confidence in the effect estimate is limited: The true effect may be substantially different from the estimate of the effect

Very low quality: We have very little confidence in the effect estimate: The true effect is likely to be substantially different from the estimate of effect

a. The methodological weaknesses in the design of included studies, relating to performance, attrition and detection bias. 


\section{Implications for research}

There is a need for better designed RCTs to determine the effectiveness of mifamurtide in the treatment of high-grade non-metastatic and metastatic osteosarcoma. The included studies lacked the information on any differences in osteosarcoma between the patient's age and its treatment, particularly relating to the dose of mifamurtide, as all patients received the same dose of mifamurtide, regardless of their age. This review recommends that future research should consider subgroup analysis by age. Furthermore, the importance of measuring HRQoL should be considered as it would be essential for cost-analysis studies and impact on the recommendation (or not) of mifamurtide. This review was based on existing trials with methodological flaws, including allocation concealment and blinding. In one of the included papers identified in this review the terms "hazard ratio for overall survival" and "relative risk of death" were used interchangeably. The survival measures of treatment effects such as odds ratios (ORs), relative risks (RRs), and hazard ratio (HR) are commonly used in prospective clinical trials, however a common mistake is to interpret RRs instead of HRs. ${ }^{83-85}$ Therefore, this review recommends that any future research should avoid any inappropriate use of statistical interpretation, while assessing differences between two similar survival curves. Secondly, the proportional hazard regression model for analyses of censored survival data which gives rise to HRs also needs to be correctly interpreted. ${ }^{85,86}$ The limitations of the current studies should be thoroughly considered in the design of future clinical trials. High quality research studies that clearly involve a larger sample size, distinguishing non-metastatic and metastatic populations, are required. Efforts should be made to have consistency in terminology and their definitions used to interpret outcomes such as PFS or progression-free interval or disease-free survival, and survival after relapse. This is critical to facilitate comparison and enhance the opportunity for possible future meta-analysis that can draw more reliable conclusions. Additionally, to ensure high quality studies, future RCTs should follow the desiderata for survival analysis. ${ }^{87}$ Some of the desiderata 87 include: (1) a well-defined event in a clear and unambiguous way; (2) a justified explanation of sampling method and sample size; (3) summary of statistics of measured variables; (4) presentation of graphs of the survivor function(s); (5) presentation of hazard ratio together with confidence intervals and p-values; (6) appropriate addressing of the treatment of missing data; and (7) addressing details on censoring. Survival analysis mainly features the true ability to handle the right censoring, which only occurs when some patients do not experience any events. ${ }^{87}$ All three included papers did mention censored patients, who were without any events at date of the last contact, but the actual data was not clearly reported. Hence, the desiderata for survival analysis should be considered in future research to ensure high quality studies. 


\section{CONFLICT OF INTEREST}

There are no conflicts of interest to declare by any of the authors. 


\section{REFERENCES}

1. Chou AJ, Kleinerman ES, Krailo MD, Chen Z, Betcher DL, Healey JH, et al. Addition of muramyl tripeptide to chemotherapy for patients with newly diagnosed metastatic osteosarcoma: a report from the Children's Oncology Group. Cancer 2009; 115(22): 5339-5348.

2. Meyers PA, Schwartz CL, Krailo MD, Healey JH, Bernsein ML, Betcher D, et al. Osteosarcoma: the addition of muramyl tripeptide to chemotherapy improves overall survival--a report from the Children's Oncology Group. J Clin Oncol 2008; 26(4): 633-638.

3. Kleinerman ES, Gano JB, Johnston DA, Benjamin RS, Jaffe N. Efficacy of liposomal muramyl tripeptide (CGP 19835A) in the treatment of relapsed osteosarcoma. Am J Clin Oncol 1995; 18(2): 93-99.

4. Grabowski P. Physiology of bone. Endocr Dev 2009; 16: 32-48.

5. Maya K, Michele WT, Mark JS, David MT. Translational biology of osteosarocma. Nature Reviews Cancer 2014; 14: 722-735.

6. Mutsaers AJ, Walkley CR. Cells of origin in osteosarcoma: mesenchymal stem cells or osteoblast committed cells? Bone. 2014; 62: 56-63.

7. Roodman GD. Cell biology of the osteoclast. Exp Hematol 1999; 27(8): 1229-1241.

8. Majo J, Cubedo R, Pardo A. Treatment of Osteosarcoma. A Review. Rev Esp Cir Orthop Traumatol 2010; 54(5): 329-336.

9. Athanasou N, Bielack S, de Alava E, Tos APD, Ferrari S, Gelderblom H, et al. Bone sarcomas: ESMO clinical practice guidelines for diagnosis, treatment and follow-up. Annals of Oncol 2010; 21: v204-v213.

10. Anderson P. Liposomal muramyl tripeptide phosphatidyl ethanolamine: ifosfamidecontaining chemotherpay in osteosarcoma. Future Oncol 2006; 2(3): 333-343.

11. Gill J, Ahluwalia MK, Geller D, Gorlick R. New targets and approaches in osteosarcoma. Pharmacol Ther 2013; 137(1): 89-99.

12. Marina N, Gebhardt M, Teot L, Gorlick R. Biology and therapeutic advances for pediatric osteosarcoma. The Oncologist 2004; 9(4): 422-441.

13. Kundu ZS. Classification, imaging, biopsy and staging of osteosarcoma. Indian J Orthopaedics 2014; 48(3): 238-246.

14. Sakamoto A, Iwamoto Y. Current Status and Perspectives Regarding the Treatment of Osteosarcoma: Chemotherapy. Reviews on Recent Clinical Trials 2008; 3(3): 228-231.

15. Chou AJ, Gorlick R. Chemotherapy resistance in osteosarcoma: Current challenges and future directions. Expert Rev Anticancer Ther 2006; 6(7): 1075-1085.

16. Mori K, Ando K, Heymann D. Liposomal muramyl tripeptide phosphatidyl ethanolamine: a safe and effective agent against osteosarcoma pulmonary metastases. Expert Rev Anticancer Ther 2008; 8(2): 151-159.

17. Kager L, Pötschger U, Bielack S. Review of mifamurtide in the treatment of patients with osteosarcoma. Ther Clin Risk Manag 2010; 6: 279-286.

18. Meyers PA, Gorlick R. Osteosarcoma. Pediatr Clin North Am 1997; 44(4): 973-989.

19. $\quad$ Ritter J, Bielack SS. Osteosarcoma. Ann Oncol 2010; 21(suppl 7): vii320-vii325.

20. The ESMO/European Sarcoma Network Working Group. Bone sarcomas: ESMO clinical practice guidelines for diagnosis, treatment and follow-up. Ann Oncol 2012; 23(suppl 7): vii100vii109.

21. Redondo A, Cruz J, Lopez-Pousa A. SEOM clinical guidlines for the treatment of osteosarcoma in adults-2013. Clin Transl Oncol 2013; 15(12): 1037-1043.

22. Luetke A, Meyer PA, Lewis I, Juergens H. Osteosarcoma treatment - where do we stand? A state of the art review. Cancer Treat Rev 2014; 40(4): 523-532. 
23. Bielack S, Carrle D, Casali PG. Osteosarcoma: ESMO clinical recommendations for diagnosis, treatment and follow-up. Annals of Oncol 2009; 20(suppl 4): iv137-iv139.

24. Frampton JE. Mifamurtide: a review of its use in the treatment of osteosarcoma. Paediatr Drugs 2010; 12(3): 141-153.

25. Liposomal muramyl tripeptide phosphatidyl ethanolamine (Mepact) for osteosarcoma: horizon scanning technology briefing (Structured abstract). Heatlh Technology Assessment Database. National Horizon Scanning Centre (NHSC) 2006; (3) 1-5. Available from URL: http://onlinelibrary.wiley.com/o/cochrane/clhta/articles/HTA-32007000150/frame.html.

26. Johal S, Ralston S, Knight C. Mifamurtide for high-grade, resectable, nonmetastatic osteosarcoma following surgical resection: a cost-effectiveness analysis (Provisional abstract). Value Health 2013; 16(8): 1123-1132.

27. Bielack SS, Carrle D, Hardes J, Schuck A, Paulussen M. Bone tumors in adolescents and young adults. Curr Treat Options in Oncol 2008; 9(1): 67-80.

28. Ando K, Mori K, Corradini N, Redini F, Heymann D. Mifamurtide for the treatment of nonmetastatic osteosarcoma. Expert Opin Pharmacother 2011; 12(2): 285-292.

29. Meyer PA. Muramyl tripeptide (mifamurtide) for treatment of osteosarcoma. Expert Rev Anticancer Ther 2009; 9(8): 1035-1049.

30. Understanding Primary Bone Cancer information fact sheet, Cancer Council Australia. Medical Reveiw of source booklet. June 2015.

31. Meyers PA, Schwartz CL, Krailo M, Kleinerman ES, Betcher D, Bernstein ML, et al. Osteosarcoma: a randomized, prospective trial of the addition of ifosfamide and/or muramyl tripeptide to cisplatin, doxorubicin, and high-dose methotrexate. J Clin Oncol 2005; 23(9):2004-2011.

32. Abrams AK, Avedian RS, Marina N. Treating pediatric osteosarcoma: Recent clinical trial evidence. Clin Invest 2013; 3(10): 967-978.

33. Federman N, Bernthal N, Eilber FC, Tap WD. The multidisciplinary management of osteosarcoma. Curr Treat Options in Oncol 2009; 10(1-2): 82-93.

34. Ando K, Heymann MF, Stresing V, Mori K, Redini F, Heymann D. Current therapeutic strategies and novel approaches in osteosarcoma. Cancers 2013; 5(2): 591-616.

35. Song HJ, Lee E-K, Lee JA, Kim H-L, Jang KW. The addition of mifamurtide to chemotherapy improves lifetime effectiveness in children with osteosarcoma: a Markov model analysis. Tumour Biol $2014 ; 35(9): 8771-9$

36. Jaffe N. Osteosarcoma: review of the past, impact on the future. The American experience. Cancer Treat Res 2009; 152: 239-262.

37. Endo-Munoz L, Evdokiou A, Saunders NA. The role of osteoclasts and tumour-associated macrophages in osteosarcoma metastasis. Biochim Biophys Acta 2012; 1826(2): 434-442.

38. Loeb DM. Is There a Role for Immunotherapy in Osteosarcoma? In: Jaffe N, Bruland OS, Bielack SS, (eds). Pediatric and Adolescent Osteosarcoma. Cancer Treat Res. Dordrecht: Springer, 2009; 447-457.

39. Marina N, Gorlick R. Immune approaches to treating osteosarcoma. Cancer Biol \& Ther 2009; 8(10): 981-983.

40. Kleinerman ES, Raymond AK, Bucana CD, JaffN, Harris MB, Krakoff IH, et al. Unique histological changes in lung metastases of osteosarcoma patients following therapy with liposomal muramyl tripeptide (CGP-19835A lipid). Cancer Immunol Immunother 1992; 34(4): 211-220.

41. Killion JJ, Kleinerman ES, Wilson MR, Tanaka M, Fidler IJ. Sequential therapy with chemotherapeutic drugs and liposome-encapsulated muramyl tripeptide: determination of potential interactions between these agents. Oncol Res 1992; 4(10): 413-418.

42. Buddingh EP, Kuijjer ML, Duim R, Burger H, Agelopoulos K, Myklebost O, et al. Tumorinfiltrating macrophages are associated with metastasis suppression in high-grade osteosarcoma: $\mathrm{A}$ rationale for treatment with macrophage-activating agents. Clin Cancer Res 2011; 17(8): 2110-2119. 
43. Romet-Lemonne J-L, Mills B, Fridman WH, Munsell M. Prospectively planned analysis of data from a phase III study of liposomal muramyltripeptide phosphatidylethanolamine in the treatment of osteosarcoma. J Clin Oncol 2005; 23(26): 6437-6438.

44. Cleton-Jansen A-M, Buddingh EP, Lankester AC. Immunotherapy Is it different for sarcomas? Oncolmmunology 2012; 1(2): 255-257.

45. Mifamurtide - CGP 19835, CGP 19835A, L-MTP-PE, liposomal MTP-PE, MLV 19835A, MTP-PE, muramyltripeptide phosphatidylethanolamine. Drugs R D 2008; 9(2): 131-135.

46. Gano J, Kleinerman E. Liposomal MTP-PE: A Promising New Biologic Response Modifier. Oncol Nurs Forum 1995; 22: 809-816.

47. Nardin A, Lefebvre ML, Labroquère K, Faure O, Abastado JP. Liposomal muramyl tripeptide phosphatidylethanolamine: Targeting and activating macrophages for adjuvant treatment of osteosarcoma. Curr Cancer Drug Targets 2006; 6(2): 123-133.

48. Asano T, Kleinerman ES. Liposome-encapsulated MTP-PE: A novel biologic agent for cancer therapy. J Immunother Emphasis Tumor Immunol. 1993; 14(4): 286-292.

49. Meyers P, Schwartz C, Bernstein M, Betcher D, Conrad E, Ferguson W, et al. Addition of ifosfamide and muramyl tripeptide to cisplatin, doxorubicin and high-dose methotrexate improves event free survival (EFS) in localized osteosarcoma (OS) [abstract1463]. 2001. Proceedings of ASCO 2001; 20(Pt 1): 367a.

50. Kleinerman ES, Jia SF, Griffin J, Seibel NL, Benjamin RS, Jaffe N. Phase-II Study of Liposomal Muramyl Tripeptide in Osteosarcoma: The Cytokine Cascade and Monocyte Activation Following Administration. J Clin Oncol 1992; 10(8): 1310-1316.

51. Meyers PA, Chou AJ. Muramyl tripeptide-phosphatidyl ethanolamine encapsulated in liposomes (L-MTP-PE) in the treatment of osteosarcoma. Adv Exp Med Biol 2014; 804: 307-321.

52. Matthay KK. Osteosarcoma. Evidence-Based Pediatric Oncology: Third Edition: John Wiley and Sons, 2013; 14-24.

53. Chonn A, Cullis PR. Recent advances in liposomal drug-delivery systems. Current Biology : CB 1995; 6(6): 698-708.

54. Kleinerman ES, Snyder A, Jaffe N. Influence of Chemotherapy Administration on Monocytes Activation by Liposomal Muramyl Tripeptide Phosphatidylethanolamine in Children With Osteosarcoma. J Clin Oncol 1991; 9(2): 259-267.

55. Gano JB, Kleinerman ES. Liposomal MTP-PE. J Pediatr Oncol Nurs 1994; 11(4): 161-163.

56. Anderson P, Meyers P, Kleinerman E, Oliva C, Liu Y. Mifamurtide (I-mtp-pe) for metastatic and recurrent osteosarcoma (OS): survival and safety profile from a patient access study[ix488/abstracts 1508P]. Ann Oncol 2012; 23: 488.

57. Fidler IJ, Kleinerman ES. Clincial application of phospholipid liposomes containing macrophage activators for therapy of cancer metastasis. Adv Drug Delivery Rev 1994; 13(3): 325340.

58. Bramwell V. Metastatic ostoesarcoma: a review of current issues in systemic treatment. Sarcoma 1997; 1(3-4): 123-130.

59. Kleinerman ES, Meyers PA, Raymond AK, Gano JB, Jia SF, Jaffe N. Combination therapy with ifosfamide and liposome-encapsulated muramyl tripeptide: tolerability, toxicity, and immune stimulation. J Immunother 1995; 17(3): 181-193.

60. Anderson PM, Meyers P, Kleinerman E, Venkatakrishnan K, Hughes DP, Herzog C, et al. Mifamurtide in Metastatic and recurrent osteosarcoma: A patient access study with pharmacokinetics, pharmacodynamic, and safety assessments. Pediatr Blood Cancer 2014; 61(2): 238-244.

61. Pearson A, Wiechula R, Court A, Lockwood C. The JBI model of evidence-based healthcare. Int J Evid Based Healthc 2005; 3(8): 207-215. 
62. The Joanna Briggs Institute. 2014. Joanna Briggs Institute Reviewers' Manual. 2014 edition. Australia: The Joanna Briggs Institute.

63. Sackett DL, Rosenberg WMC, Gray JAM, Haynes RB, Richardson WS. Evidence based medicine: what it is and what it isn't: it's about integrating individual clinical expertise and the best external evidence. BMJ 1996; 312: 71-72.

64. Lee K. The Philosophical Foundations of Modern Medicine: Palgrave Macmillan UK 2011.

65. Jordan Z, Lockwood C, Aromataris E, Munn Z. The updated JBI Model of Evidence-based Healthcare: A Model Reconsidered. The Joanna Briggs Institute 2016.

66. Pearson A. Evidence synthesis and its role in evidence-based health care. Nurs Clin North Am 2014; 49(4): 453-460.

67. The Joanna Briggs Institute. 2011. Joanna Briggs Institute Reviewers' Manual. 2011 edition. Australia: The Joanna Briggs Institute.

68. Grant MJ, Booth G. A typology of reviews: an analysis of 14 review types and associated methodologies. Health Info Libr J. 2009; 26(2): 91-108.

69. The Joanna Briggs Institute. 2015. Joanna Briggs Institute Reviewers' Manual. 2015 edition. Australia: The Joanna Briggs Institute.

70. Armstrong R, Hall BJ, Doyle J, Waters E. Cochrane update: 'Scoping the scope' of a cochrane review. J Public Health (Oxf). 2011; 33(1): 147-150.

71. Saso S, Panesar P, Weiming S, Athanasiou T. Systematic review and metaanalysis in clinical practice In: Athanasiou T, Darzi A, editors. Evidence Synthesis in Healthcare. London: Springer 2011: 67-114.

72. Jimmy R, White S, Lisy K. Effectiveness of mifamurtide in addition of standard chemotherapy for high-grade osteosarcoma: a systematic review protocol. JBI Database System Rev Implement Rep. 2014; 12(11): 61-73.

73. Higgins JPT, Green S (editors). Cochrane Handbook for Systematic Reviews of Interventions Version 5.1.0 [updated March 2011]. The Cochrane Collaboration 2011. Available from: https://www.cochrane-handbok.org.

74. Munn Z, Porritt K, Aromataris E, Lockwood C, Peters M. The Joanna Briggs Institute Levels of Evidence and Grades of Recommendation Working Party. Summary of Findings Tables for Joanna Briggs Institute Systematic Reviews. . The Joanna Briggs Institute 2016.

75. Joanna Briggs Institute (2014). Grades of Recommendation Accessed 29 Nov 2015. Available from: http://joannabriggs.org/jbi-approach.html-tabbed-nav=Grades-of-Recommendation.

76. Anderson P, Bloodworth L, Anna S, Salazar-Abshire M, Tomaras M, Salvador LK. Few infusion related side effects after mifamurtide[SIOP abstracts PD007]. Pediatr. Blood Cancer 2010; 55(5): 876.

77. Kebudi R, Ayan I, Ozger H, Cakir FB, Gorgun O, Bay SB, et al. Efficacy in six courses of nonmethotrexate three-drug chemotherapy and surgery in osteosarcoma: 25-year experience (Supplement). J of Clin Oncol 2015; 33(15): 10050.

78. Dzierzbicka K, Gozdowska M, Kolodziejczyk AM. [L-MTP-PE--a potential antineoplastic agent]. Postepy Hig Med Dosw 1997; 51(2): 227-236.

79. Porter JB, Desmond M, O'Donnell M, McNamara P, Lehnert M, Wang B, et al. Challenges of a post-authorisation safety study (PASS) in an orphan oncology indication. Pharmacoepidemiology and Drug Safety 2014; 23(S1): 1 - 497.

80. Lamplot JD, Denduluri S, Qin J, Li R, Liu X, et al. The Current and Future Therapies for Human Osteosarcoma. Curr Cancer Ther Rev 2013; 9(1): 55-77.

81. Therapeutic Goods Administration. Department of Health. 2016; [internet]. Available from: https://www.tga.gov.au/node/3390.

82. Therapeutic Goods Regulation 1990. Federal Register of Legislative Instruments F2016C00154 2016: 1-236. 
83. Case LD, Kimmick G, Paskett ED, Lohnman K, Tucker R. Interpreting Measures of Tretment Effect in Cancerr Clinical Trials. Oncologist 2002; 7(3): 181-187.

84. Blagoev KB, Wilkerson J, Fojo T. Hazard ratios in cancer clinical trials - a primer Nat Rev Clin Oncol 2012 Jan 31; 9(3): 178-183.

85. Spruance S, Reid JE, Grace M, Samore M. Hazard Ratio in Clinical Trials. Antimicrob Agents Chemother 2004 Aug;48(8): 2787-2792.

86. Cantor AB. Understanding survival analysis. Evidence-based Oncology 2000; 1(2): 38-40.

87. Hancock GR, Mueller RO. The Reviewer's Guide to Quantitative Methods in the Social Sciences. New York: Routledge, 2010: 1-432. 


\section{APPENDICES}

\section{Appendix I: JBI Levels of evidence}

\section{LEVELS OF EVIDENCE FOR EFFECTIVENESS}

\section{Level 1 - Experimental Designs}

Level 1.a - Systematic review of Randomized Controlled Trials (RCTs)

Level 1.b - Systematic review of RCTs and other study designs

Level 1.c-RCT

Level 1.d - Pseudo-RCTs

\section{Level 2 - Quasi-experimental Designs}

Level 2.a - Systematic review of quasi-experimental studies

Level 2.b - Systematic review of quasi-experimental and other lower study designs

Level 2.c - Quasi-experimental prospectively controlled study

Level 2.d - Pre-test - post-test or historic/retrospective control group study

\section{Level 3 - Observational - Analytic Designs}

Level 3.a - Systematic review of comparable cohort studies

Level 3.b - Systematic review of comparable cohort and other lower study designs

Level 3.c - Cohort study with control group

Level 3.d - Case - controlled study

Level 3.e - Observational study without a control group

\section{Level 4 - Observational - Descriptive studies}

Level 4.a - Systematic review of descriptive studies

Level $4 . b$ - Cross-sectional study

Level 4.c-Case series

Level $4 . d$ - Case study

\section{Level 5 - Expert Opinion and Bench Research}

Level 5.a - Systematic review of expert opinion

Level 5.b - Expert consensus

Level 5.c-Bench research single expert opinion 


\section{Appendix II: New JBI Grades of Recommendation}

\begin{tabular}{|l|l|}
\hline \multicolumn{2}{|l|}{ JBI Grades of Recommendation } \\
\hline Grade A & $\begin{array}{l}\text { A 'strong' recommendation for a certain health management strategy where (1) it is } \\
\text { clear that desirable effects outweigh undesirable effects of the strategy; (2) where } \\
\text { there is evidence of adequate quality supporting its use; (3) there is a benefit or no } \\
\text { impact on resource use, and (4) values, preference and the patient experience } \\
\text { have been taken into account. }\end{array}$ \\
\hline Grade B & $\begin{array}{l}\text { A 'weak' recommendation for a certain health management strategy where (1) } \\
\text { desirable effects appear to outweigh undesirable effects of the strategy, although } \\
\text { this is not as clear; (2) where there is evidence supporting its use, although this } \\
\text { may not be of high quality; (3) there is a benefit, no impact or minimal impact on } \\
\text { resource use, and (4) values, preferences and the patient experience may or may } \\
\text { not have been taken into account. }\end{array}$ \\
\hline
\end{tabular}

The FAME (Feasibility, Appropriateness, Meaningfulness and Effectiveness) scale may help inform the wording and strength of a recommendation.

F - Feasibility:

$>$ What is the cost effectiveness of the practice?

$>$ Is the resource/practice available?

$>$ Is there sufficient experience/levels of competency available?

A - Appropriateness:

Is it culturally acceptable?

$>$ Is it transferable/application to the majority of the population?

$>$ Is it easily adaptable to a variety of circumstances?

M - Meaningfulness:

Is it associated with positive experiences?

$>$ Is it not associated with negative experiences?

E - Effectiveness:

Was there a beneficial effect?

$>$ Is it safe? (i.e. is there a lack of harm associated with the practice?) 


\section{Appendix III: Search strategy}

\section{PubMed:}

Initial Search Date: 17th September 2014

Updated Search Date: 30th May 2016

Language: English

Publication year Limit: 1990-May 2016

\begin{tabular}{|c|c|c|}
\hline Search & Search parameters & Results \\
\hline$\# 1$ & $\begin{array}{l}\text { osteosarcoma[mh:noexp] OR osteosarcoma, juxtacortical[mh] OR } \\
\text { osteosarcoma*[tw] OR osteosarcoma, juxtacortical*[tw] OR osteogenic } \\
\text { sarcoma*[tw] }\end{array}$ & $\begin{array}{l}=23592 \\
+2353 \\
(25945)\end{array}$ \\
\hline \#2 & $\begin{array}{l}\text { mifamurtide[Supplementary Concept] OR mifamurtide[tw] OR } \\
\text { acetylmuramyl-alanyl-isoglutamine[mh] OR acetylmuramyl-Alanyl- } \\
\text { Isoglutamine[tw] OR muramyl tripeptide phosphatidylethanolamine[tw] } \\
\text { OR CGP-19835A[tw] OR CGP } 19835 \text { A[tw] OR MLV 19835[tw] OR L- } \\
\text { MTP-PE[tw] }\end{array}$ & $\begin{array}{l}=1917+48 \\
(1965)\end{array}$ \\
\hline$\# 3$ & \#1 AND \#2 & $55+10(65)$ \\
\hline
\end{tabular}

NB: Results in brackets represent initial and updated results combined

\section{CINAHL:}

Initial Search Date: $17^{\text {th }}$ September 2014

Updated Search Date: 30th May 2016

Language: English

Publication year Limit: 1990-2016

\begin{tabular}{|c|c|c|}
\hline Search & Search parameters & Results \\
\hline$\# 1$ & $\begin{array}{l}\text { MH osteosarcoma+ OR MM osteosarcoma drug therapy OR TI } \\
\text { osteosarcoma* OR AB osteosarcoma* OR TI osteogenic, sarcoma* } \\
\text { OR AB osteogenic, sarcoma* OR TI osteosarcoma, juxtacortical* } \\
\text { OR AB osteosarcoma, juxtacortical* }\end{array}$ & $\begin{array}{l}=289+155 \\
(344)\end{array}$ \\
\hline \#2 & $\begin{array}{l}\text { MH biological response modifiers+ OR TI biological response } \\
\text { modifiers* OR AB biological response modifiers* OR TI mifamurtide } \\
\text { OR * AB mifamurtide* OR TI acetylmuramyl-Alanyl-Isoglutamine* } \\
\text { OR AB acetylmuramyl-Alanyl-Isoglutamine* OR TI muramyl } \\
\text { tripeptide phosphatidylethanolamine OR AB muramyl tripeptide } \\
\text { phosphatidylethanolamine* TI CGP-19835A* OR AB CGP-19835A* }\end{array}$ & $\begin{array}{l}=582+38 \\
(620)\end{array}$ \\
\hline
\end{tabular}




\begin{tabular}{|l|l|l|}
\hline & $\begin{array}{l}\text { OR TI CGP 19835 A } \\
\text { AB MLV AB CGP 19835 A* OR TI MLV 19835* OR }\end{array}$ & \\
\hline$\# 3$ & $\# 1$ AND \#2 & $=4+1(5)$ \\
\hline
\end{tabular}

NB: Results in brackets represent initial and updated results combined

\section{Embase}

Initial Search Date: 14th September 2014

Updated Search Date: $5^{\text {th }}$ June 2016

Language: English

Publication year Limit: 1990-2016

\begin{tabular}{|l|l|l|}
\hline Search & Search parameters & Results \\
\hline$\# 1$ & 'osteosarcoma'/syn OR osteosarcoma*:ab,ti & $=22913$ \\
& & +35139 \\
& & $(58052)$ \\
\hline$\# 2$ & 'mifamurtide'/syn OR 'muramyl tripeptide'/exp OR 'muramyl & $=1353+1623$ \\
& tripeptide':ab OR 'muramyltripeptide':ab,ti OR 'mtp-pe':ab,ti & $(2976)$ \\
\hline$\# 3$ & \#1 AND \#2 AND [english]/lim AND ([embase]/lim NOT [medline]/lim) & $=66+38$ (104) \\
& NOT [5-6-2016]/sd AND [1990-2016]/py & \\
\hline
\end{tabular}

NB: Results in brackets represent initial and updated results combined

\section{Cochrane Library}

Initial Search Date: $17^{\text {th }}$ September 2014

Updated Search Date: $5^{\text {th }}$ June 2016

Language: English

Publication year Limit: 1990-2016

\begin{tabular}{|l|l|l|}
\hline Search & Search parameters & Results \\
\hline$\# 1$ & $\begin{array}{l}\text { osteosarcoma* OR osteosarcoma, juxtacortical* OR "osteogenic } \\
\text { sarcoma*" }\end{array}$ & $=264+44$ (308) \\
\hline \#2 & $\begin{array}{l}\text { mifamurtide OR "acetylmuramyl-Alanyl-Isoglutamine" OR "muramyl } \\
\text { tripeptide phosphatidylethanolamine" OR "CGP-19835A" OR "CGP } \\
19835 \text { A" OR "MLV 19835" OR "L-MTP-PE" OR "muramyl tripeptide" } \\
\text { OR "biological response modifier*" }\end{array}$ & $=172+30(202)$ \\
\hline
\end{tabular}




\begin{tabular}{|l|l|l|}
\hline$\# 3$ & $\# 1$ AND \#2 & $10+1(11)$ \\
\hline
\end{tabular}

NB: Results in brackets represent initial and updated results combined

\section{Scopus:}

Initial Search Date: 14th September 2014

Updated Search Date: $5^{\text {th }}$ June 2016

Language: English

Publication year Limit: 1990- 2016

\begin{tabular}{|c|c|c|}
\hline Search & Search parameters & Results \\
\hline$\# 1$ & $\begin{array}{l}\text { osteosarcoma* OR \{osteosarcoma, juxtacortical\} }{ }^{*} \text { OR \{osteogenic } \\
\text { sarcoma }\}^{*}\end{array}$ & $\begin{array}{l}=31468+4175 \\
(35643)\end{array}$ \\
\hline \#2 & $\begin{array}{l}\text { Mifamurtide OR \{acetylmuramyl-Alanyl-Isoglutamine\} OR \{muramyl } \\
\text { tripeptide phosphatidylethanolamine\} OR \{CGP-19835A\} OR \{CGP } \\
19835 \text { A\} OR \{MLV 19835\} OR \{L-MTP-PE\} OR \{muramyl tripeptide }\end{array}$ & $\begin{array}{l}=2366+198 \\
(2564)\end{array}$ \\
\hline \#3 & \#1 AND \#2 & $154+40(194)$ \\
\hline
\end{tabular}

NB: Results in brackets represent initial and updated results combined

\section{Web of Science:}

Initial Search Date: $13^{\text {th }}$ September 2014

Updated Search Date: $5^{\text {th }}$ June 2016

Language: English

Publication year Limit: 1990-2016

\begin{tabular}{|l|l|l|}
\hline Search & Search parameters & Results \\
\hline$\# 1$ & TOPIC:((osteosarcoma* OR osteosarcoma juxtacortical $\left.{ }^{*}\right) \quad$ OR & $=18138+1647$ \\
& Osteogenic sarcoma* $^{*}$ & $5(34613)$ \\
\hline$\# 2$ & TOPIC:((((((((mifamurtide OR acetylmuramyl-Alanyl-Isoglutamine) & $=2289+383$ \\
& OR muramyl tripeptide phosphatidylethanolamine) OR CGP-19835A) & $(2672)$ \\
& OR CGP 19835a) OR MLV 19835a) OR L-MTP-PE) OR muramyl & \\
& tripeptide) OR biological response modifier $\left.{ }^{*}\right)$ & \\
\hline$\# 3$ & $\# 1$ AND \#2 & $139+54(193)$ \\
\hline
\end{tabular}

NB: Results in brackets represent initial and updated results combined 


\section{Cancerlit:}

Initial Search Date: $13^{\text {th }}$ September 2014

Updated Search Date: $5^{\text {th }}$ June 2016

Language: English

Publication year Limit: 1990-2016

\begin{tabular}{|l|lrr|l|}
\hline Search & Search parameters & Results \\
\hline$\# 1$ & (osteosarcoma OR osteogenic sarcoma) & AND (mifamurtide OR & $0+0(0)$ \\
& acetylmuramyl-Alanyl-Isoglutamine OR & muramyl tripeptide & \\
& phosphatidylethanolamine OR CGP-19835A OR CGP 19835a A OR & \\
& MLV 19835a OR L-MTP-PE OR muramyl tripeptide OR biological & \\
& response modifier) & & \\
\hline
\end{tabular}

NB: Results in brackets represent initial and updated results combined

\section{Grey literature:}

\section{Google advance search:}

Initial Search Date: $17^{\text {th }}$ September 2014

Updated Search Date: $5^{\text {th }}$ June 2016

Language: English

Publication year Limit: 1990-2016

\begin{tabular}{|l|l|l|}
\hline Search & Search parameters & $\begin{array}{l}\text { Results in } \\
\text { Copub }\end{array}$ \\
\hline$\# 1$ & $\begin{array}{l}\text { (osteosarcoma OR osteogenic sarcoma) AND (mifamurtide OR } \\
\text { acetylmuramyl-Alanyl-Isoglutamine OR muramyl tripeptide } \\
\text { phosphatidylethanolamine OR CGP-19835A OR CGP 19835a A OR } \\
\text { MLV 19835a OR L-MTP-PE OR muramyl tripeptide OR biological } \\
\text { response modifier) }\end{array}$ & \\
\hline
\end{tabular}

NB: Results in brackets represent initial and updated results combined

\section{MedNar}

Initial Search Date: 16 ${ }^{\text {th }}$ September 2014

Updated Search Date: $9^{\text {th }}$ June 2016

Language: English

Publication year Limit: 1990-2016 


\begin{tabular}{|l|l|l|}
\hline Search & Search parameters & Results \\
\hline$\# 1$ & (osteosarcoma OR osteosarcoma, Juxtacortical OR osteogenic & $2704+330$ \\
& sarcoma) AND (mifamurtide OR acetylmuramyl-Alanyl-Isoglutamine & $(3034)$ \\
& OR muramyl tripeptide phosphatidylethanolamine OR CGP-19835A & \\
& OR CGP 19835a A OR MLV 19835a OR L-MTP-PE OR muramyl \\
& tripeptide OR biological response modifier) & \\
\hline \multicolumn{2}{|l}{ Duplicates removed from \#1 } & $880+15$ \\
\multicolumn{2}{|l}{ Remainder \#1 } & $(895)$ \\
\hline
\end{tabular}

NB: Results in brackets represent initial and updated results combined

\section{Clinical trial gov:}

Initial Search Date: 13 ${ }^{\text {th }}$ September 2014

Updated Search Date: $10^{\text {th }}$ June 2016

Language: English

Publication year Limit: 1990-2016

\begin{tabular}{|c|c|c|}
\hline Search & Search parameters & Results \\
\hline$\# 1$ & $\begin{array}{l}\text { (osteosarcoma OR osteogenic sarcoma) AND (mifamurtide OR } \\
\text { acetylmuramyl-Alanyl-Isoglutamine OR muramyl tripeptide } \\
\text { phosphatidylethanolamine OR CGP-19835A OR CGP 19835a A OR } \\
\text { MLV 19835a OR L-MTP-PE OR muramyl tripeptide OR biological } \\
\text { response modifier) }\end{array}$ & $4+0(4)$ \\
\hline
\end{tabular}

NB: Results in brackets represent initial and updated results combined

\section{National Library of Medicine:}

Initial Search Date: 17th September 2014

Updated Search Date: 10th June 2016

Language: English

Publication year Limit: 1990-2016

\begin{tabular}{|l|lrl|l|}
\hline Search & Search parameters & Results \\
\hline$\# 1$ & (osteosarcoma OR osteogenic sarcoma) AND (mifamurtide OR & $5+5(10)$ \\
& acetylmuramyl-Alanyl-Isoglutamine OR muramyl tripeptide & \\
& phosphatidylethanolamine OR CGP-19835A OR CGP 19835a A OR & \\
& MLV 19835a OR L-MTP-PE OR muramyl tripeptide OR biological & \\
\hline
\end{tabular}


response modifier)

NB: Results in brackets represent initial and updated results combined

National Comprehensive Cancer Network: www.ncen.org

Initial Search Date: 17th September 2014

Updated Search Date: $10^{\text {th }}$ June 2016

Language: English

Publication year Limit: 1990-2016

\begin{tabular}{|l|lrr|l|}
\hline Search & Search parameters & Results \\
\hline$\# 1$ & (osteosarcoma OR osteogenic sarcoma) & AND (mifamurtide OR & $0+0(0)$ \\
& acetylmuramyl-Alanyl-Isoglutamine OR $\quad$ muramyl tripeptide & \\
& phosphatidylethanolamine OR CGP-19835A OR CGP 19835a A OR & \\
& $\begin{array}{l}\text { MLV 19835a OR L-MTP-PE OR muramyl tripeptide OR biological } \\
\text { response modifier) }\end{array}$ & \\
\hline
\end{tabular}

NB: Results in brackets represent initial and updated results combined

\section{National Cancer Institute: www.cancer.gov}

Initial Search Date: 17th September 2014

Updated Search Date: 10 $10^{\text {th }}$ June 2016

Language: English

Publication year Limit: 1990-2016

\begin{tabular}{|l|lrr|l|}
\hline Search & Search parameters & Results \\
\hline$\# 1$ & (osteosarcoma OR osteogenic sarcoma) AND (mifamurtide OR & $1+0(1)$ \\
& $\begin{array}{l}\text { acetylmuramyl-Alanyl-Isoglutamine OR } \\
\text { phosphatidylethanolamine OR CGP-19835A OR CGP 19835a A OR }\end{array}$ & \\
& MLV 19835a OR L-MTP-PE OR muramyl tripeptide) & \\
\hline
\end{tabular}

NB: Results in brackets represent initial and updated results combined

\section{CureSearch: www.curesearch.org}

Initial Search Date: 13 $3^{\text {th }}$ September 2014

Updated Search Date: $10^{\text {th }}$ June 2016

Language: English

Publication year Limit: 1990-2016

Search Search parameters

Results 


\begin{tabular}{|l|lrrr|l|}
\hline$\# 1$ & (osteosarcoma OR osteogenic sarcoma) & AND & (mifamurtide OR & $0+235$ \\
& acetylmuramyl-Alanyl-Isoglutamine & OR & muramyl tripeptide & $(235)$ \\
phosphatidylethanolamine OR CGP-19835A & OR CGP 19835a A OR & \\
& $\begin{array}{l}\text { MLV 19835a OR L-MTP-PE OR muramyl tripeptide OR biological } \\
\text { response modifier) }\end{array}$ & \\
\hline
\end{tabular}

NB: Results in brackets represent initial and updated results combined 


\section{Appendix IV: Appraisal instrument}

\section{JBI Critical Appraisal Checklist for Randomised Control / Pseudo-randomised Trial}

Reviewer _. . . . . . . . Date

Author

Year Record Number

1. Was the assignment to treatment groups truly random?

2. Were participants blinded to treatment allocation?

3. Was allocation to treatment groups concealed from the allocator?

4. Were the outcomes of people who withdrew described and included in the analysis?

5. Were those assessing outcomes blind to the treatment allocation?

6. Were the control and treatment groups comparable at entry?

7. Were groups treated identically other than for the named interventions

8. Were outcomes measured in the same way for all groups?

9. Were outcomes measured in a reliable way?

10. Was appropriate statistical analysis used? 
Appendix V: Data extraction instrument

\section{JBI Data Extraction Form for \\ Experimental / Observational Studies}

Reviewer $\ldots \ldots \ldots$ Date $\ldots \ldots \ldots$
Author $\ldots \ldots \ldots \ldots$
Journal_ $\ldots \ldots \ldots \ldots$

Study Method

RCT

Quasi-RCT

Longitudinal

Retrospective

Observational

Other

Participants

Setting

Population

Sample size

Group A

Group B

Interventions

Intervention A

Intervention B

Authors Conclusions:

Reviewers Conclusions: 
Study results

Dichotomous data

\begin{tabular}{|l|l|l|}
\hline Outcome & $\begin{array}{c}\text { Intervention ( ) } \\
\text { number / total number }\end{array}$ & $\begin{array}{c}\text { Intervention ( ) } \\
\text { number / total number }\end{array}$ \\
\hline & & \\
\hline & & \\
\hline & & \\
\hline & & \\
\hline & & \\
\hline
\end{tabular}

Continuous data

\begin{tabular}{|l|l|l|}
\hline Outcome & $\begin{array}{c}\text { Intervention ( ) } \\
\text { number/ total number }\end{array}$ & $\begin{array}{c}\text { Intervention ( ) } \\
\text { number / total number }\end{array}$ \\
\hline & & \\
\hline & & \\
\hline & & \\
\hline & & \\
\hline & & \\
\hline
\end{tabular}




\section{Appendix VI: Studies excluded after review of full text}

\section{Excluded studies $n=71$}

1. Song HJ, Lee E-K, Lee JA, Kim H-L, Jang KW. The addition of mifamurtide to chemotherapy improves lifetime effectiveness in children with osteosarcoma: a Markov model analysis. Tumour Biol 2014.

Reason for exclusion: This paper did not address the outcome of the review as it used the Markov model to compare the expected lifetime quality-adjusted life year (QALYs) between mifamurtide along with chemotherapy versus chemotherapy alone.

2. Bone sarcomas: ESMO clinical practice guidelines for diagnosis, treatment and follow-up. Annals of Oncology 2012; 23: vii100-vii109.

Reason for exclusion: This paper was a clinical practice guideline developed by European Society for Medical Oncology (ESMO) and was not a primary research study of interest to the review.

3. Athanasou N, Bielack S, de Alava E, et al. Bone sarcomas: ESMO clinical practice guidelines for diagnosis, treatment and follow-up. Annals of Oncology 2010; 21: v204-v213.

Reason for exclusion: This paper was a clinical practice guideline developed by European Society for Medical Oncology (ESMO) and was not a primary research study of interest to the review.

4. Kundu ZS. Classification, imaging, biopsy and staging of osteosarcoma. Indian Journal of Orthopaedics 2014; 48: 238-246.

Reason for exclusion: This paper was not a primary research study and did not meet the inclusion criteria.

5. Sakamoto A, Iwamoto Y. Current Status and Perspectives Regarding the Treatment of Osteosarcoma: Chemotherapy. Reviews on Recent Clinical Trials 2008; 3: 228-231.

Reason for exclusion: This paper was a literature review and not a primary research with the outcome of interest to the review.

6. Marina N, Gorlick R. Immune approaches to treating osteosarcoma. Cancer Biology \& Therapy 2009; 8: 981-983.

Reason for exclusion: This paper was not a primary research study and did not meet the inclusion criteria.

7. Cleton-Jansen A-M, Buddingh EP, Lankester AC. Immunotherapy. Oncolmmunology 2012; 1: 255-257.

Reason for exclusion: This paper was not a primary research study and did not meet the inclusion criteria. 
8. Pahl, J. HW, Kwappenberg, K. MC, Varypataki, E. M, Santos, S.J, Kuijjer, M. L, Mohamed, S, Wijnen, J. T, Tol, M. JD. V, Cleton-Jansen, AM, Egeler, R. M. Macrophages inhibit human osteosarcoma cell growth after activation with the bacterial cell wall derivative liposomal muramyl tripeptide in combination with interferon- $y$. Journal Experimental \& Clincial Cancer Research 2014; 33:1-13.

Reason for exclusion: This paper was not a primary research study and did not meet the inclusion criteria.

9. Muramyl tripeptide-phosphatidyl ethanolamine encapsulated in liposomes (L-MTP-PE) in the treatment of osteosarcoma.

Reason for exclusion: This paper was a literature review and not a primary research with the outcome of interest to the review.

10. Osteosarcoma treatment - where do we stand? A state of the art review.

Reason for exclusion: This paper was a review article and not a primary research looking at the outcome of interest to the review.

11. Jaffe N. Osteosarcoma: review of the past, impact on the future. The American experience. Cancer Treat Res 2009; 152: 239-262.

Reason for exclusion: This paper was a literature review of sequential discoveries in osteosarcoma research and treatment, and not a primary research looking at the outcome of interest to the review.

12. Romet-Lemonne J-L, Mills B, Fridman WH, Munsell M. Prospectively planned analysis of data from a phase III study of liposomal muramyltripeptide phosphatidylethanolamine in the treatment of osteosarcoma. J Clin Oncol 2005; 23: 6437-6438.

Reason for exclusion: This was a correspondence paper and not a primary research looking for the outcome of interest to the review. This paper reports deviations in COG analysis from the prospective design of the included study.

13. Kager L, Pötschger U, Bielack S. Review of mifamurtide in the treatment of patients with osteosarcoma. Ther Clin Risk Manag 2010; 6: 279-286.

Reason for exclusion: This paper was a literature review and not a primary research looking at the outcome of interest to the review.

14. Anderson PM, Tomaras M, McConnell K. Mifamurtide in osteosarcoma--a practical review. Drugs Today (Barc) 2010; 46: 327-337.

Reason for exclusion: This paper was a literature review and not a primary research looking at the outcome of interest to the review.

15. Frampton JE. Mifamurtide: a review of its use in the treatment of osteosarcoma. Paediatr Drugs 2010; 12: 141-153. 
Reason for exclusion: This paper was a literature review on drug evaluation and not a primary research looking at the outcome of interest to the review.

16. Meyers $P$, Schwartz $C$, Bernstein $M$, et al. Addition of ifosfamide and muramyl tripeptide to cisplatin, doxorubicin and high-dose methotrexate improves event free survival (EFS) in localized osteosarcoma (OS) [abstract]. 2001. Available from URL:http://onlinelibrary.wiley.com/o/cochrane/clcentral/articles/611/CN00693611/frame.html.

Reason for exclusion: This study appeared to meet the inclusion criteria but comprised poster presentations published in abstract form and full texts were unavailable, despite contacting the authors.

17. Liposomal muramyl tripeptide phosphatidyl ethanolamine (Mepact) for osteosarcoma: horizon scanning technology briefing (Structured abstract). 2006. Available from URL: http://onlinelibrary.wiley.com/o/cochrane/clhta/articles/HTA32007000150/frame.html.

Reason for exclusion: This paper was not the actual primary research study, but a short communication paper, and which also did not meet the inclusion criteria.

18. Johal S, Ralston S, Knight C. Mifamurtide for high-grade, resectable, nonmetastatic osteosarcoma following surgical resection: a cost-effectiveness analysis (Provisional abstract). 2013.

Reason for exclusion: This paper was a provisional abstract on economic evaluation of mifamurtide treatment arm and not a primary research looking at the outcome of interest to the review.

19. Meyers PA, Schwartz CL, Krailo M, et al. Osteosarcoma: a randomized, prospective trial of the addition of ifosfamide and/or muramyl tripeptide to cisplatin, doxorubicin, and high-dose methotrexate. 2005.

Reason for exclusion: This was a potential primary research paper that reported on the preliminary results for EFS and mifamurtide-related adverse events outcomes, which were reanalysed later with an additional follow-up to amend the reports with the updated results of this trial.

20. Ando K, Heymann MF, Stresing V, Mori K, Redini F, Heymann D. Current therapeutic strategies and novel approaches in osteosarcoma. Cancers 2013; 5: 591-616.

Reason for exclusion: This paper was not a primary research study and did not meet the inclusion criteria.

21. Anderson P, Bloodworth L, Anna S, Salazar-Abshire M, Tomaras M, Salvador LK. Few infusion related side effects after mifamurtide. Pediatric Blood and Cancer 2010; 55: 876.

Reason for exclusion: This paper appeared to meet the inclusion criteria but comprised poster presentations published in abstract form and for which full texts were unavailable, despite contacting the authors. 
22. Meyers PA, Krailo M, Grier H, Bernstein M. In reply [2]. Journal of Clinical Oncology 2005; 23: 6438-6439.

Reason for exclusion: This was a correspondence paper and not a primary research looking at the outcome of interest to the review.

23. Abrams AK, Avedian RS, Marina N. Treating pediatric osteosarcoma: Recent clinical trial evidence. Clinical Investigation 2013; 3: 967-978.

Reason for exclusion: This paper was a literature review and not a primary research looking at the outcome of interest to the review.

24. Buddingh EP, Kuijjer ML, Duim R, et al. Tumor-infiltrating macrophages are associated with metastasis suppression in high-grade osteosarcoma: A rationale for treatment with macrophage-activating agents. Laboratory Investigation 2011; 91: 10A.

Reason for exclusion: This paper was a primary research study which investigated the development of metastasis by studying tumour cells and their stromal context. Henceforth, it did not meet the inclusion criteria as per the description mentioned in the published protocol.

25. Marina N, Gebhardt M, Teot L, Gorlick R. Biology and therapeutic advances for pediatric osteosarcoma. Oncologist 2004; 9: 422-441.

Reason for exclusion: This paper was a literature review which described current treatment strategies, clinical features, radiologic and diagnostic work-up, pathology, and the state of the art management for patients with osteosarcoma. Also, it was not a primary research looking at the outcome of interest to the review.

26. Fidler IJ, Kleinerman ES. Clinical-Application Of Phospholipid Liposomes Containing Macrophage Activators For Therapy Of Cancer MetastasiS. Advanced Drug Delivery Reviews 1994; 13: 325-340.

Reason for exclusion: This paper was a literature review and not a primary research looking at the outcome of interest to the review.

27. Kleinerman ES, Meyers PA, Raymond AK, Gano JB, Jia SF, Jaffe N. Combination Therapy With Ifosfamide And Liposome-Encapsulated Muramyl Tripeptide - Tolerability, Toxicity, And Immune Stimulation. Journal of Immunotherapy 1995; 17: 181-193.

Reason for exclusion: This primary research paper was not a quantitative study design but a descriptive/case series with no control group, which is not as per the description mentioned in the published protocol.

28. Sleijfer S, Gelderblom H. Current clinical trials for advanced osteosarcoma and soft tissue sarcoma. Curr Opin Oncol 2014; 26: 434-439.

Reason for exclusion: This paper was a literature review and not a primary research looking at the outcome of interest to the review. 
29. Asano T, Fujimaki W, McWatters A, An TH, Matsushima K, Kleinerman ES. Effect Of Adriamycin On Liposomal Muramyl Tripeptides Ability To Up-Regulate Monocyte Cytokine Expression. Cancer Immunology Immunotherapy 1993; 37: 408-411.

Reason for exclusion: This paper was a literature review and not a primary research looking at the outcome of interest to the review.

30. Loeb DM. Is There a Role for Immunotherapy in Osteosarcoma? In: Jaffe N, Bruland OS, Bielack SS, (eds). Pediatric and Adolescent Osteosarcoma. Dordrecht: Springer, 2009; 447457.

Reason for exclusion: This paper was not a primary research study and did not meet the inclusion criteria.

31. Asano T, Kleinerman ES. Liposome-Encapsulated Mtp-Pe - A Novel Biologic Agent For Cancer-Therapy. Journal of Immunotherapy 1993; 14: 286-292.

Reason for exclusion: This paper was a literature review and not a primary research looking at the outcome of interest to the review.

32. Mifamurtide - CGP 19835, CGP 19835A, L-MTP-PE, liposomal MTP-PE, MLV 19835A, MTP-PE, muramyltripeptide phosphatidylethanolamine. Drugs R D 2008; 9: 131-135.

Reason for exclusion: This paper was a literature review and not a primary research looking at the outcome of interest to the review.

33. Anderson P, Meyers P, Kleinerman E, Oliva C, Liu Y. Mifamurtide (L-Mtp-Pe) For Metastatic And Recurrent Osteosarcoma (Os): Survival And Safety Profile From A Patient Access Study. Annals of Oncology 2012; 23: 488-488.

Reason for exclusion: This primary research paper was not a quantitative study design but a descriptive/case series with no control group, which is not as per the description mentioned in the published protocol.

34. Meyer P. Muramyl tripeptide for treatment of osteosarcoma. Lancet Oncology 2008; 9: 207207.

Reason for exclusion: This paper was a literature review and not a primary research looking at the outcome of interest to the review.

35. Anderson P, Kopp L, Anderson N, et al. Novel bone cancer drugs: investigational agents and control paradigms for primary bone sarcomas (Ewing's sarcoma and osteosareoma). Expert Opin Investig Drugs 2008; 17: 1703-1715.

Reason for exclusion: This paper was a literature review and not a primary research looking at the outcome of interest to the review.

36. Meyers PA, Gorlick R. Osteosarcoma. Pediatr Clin North Am 1997; 44: 973-989.

Reason for exclusion: This paper was not a primary research looking at the outcome of interest to the review. 
37. Mori K, Redini F, Gouin F, Cherrier B, Heymann D. Osteosarcoma: Current status of immunotherapy and future trends (Review). Oncology Reports 2006; 15: 693-700.

Reason for exclusion: This paper was a literature review and not a primary research looking at the outcome of interest to the review.

38. Kleinerman ES, Jia SF, Griffin J, Seibel NL, Benjamin RS, Jaffe N. Phase-li Study Of Liposomal Muramyl Tripeptide In Osteosarcoma - The Cytokine Cascade And Monocyte Activation Following Administration. Journal of Clinical Oncology 1992; 10: 1310-1316.

Reason for exclusion: This primary research paper was not a quantitative study design but a descriptive/case series with no control group, which is not as per the description mentioned in the published protocol.

39. Botter SM, Neri D, Fuchs B. Recent advances in osteosarcoma. Current Opinion in Pharmacology 2014; 16: 15-23.

Reason for exclusion: This paper was a literature review and not a primary research looking at the outcome of interest to the review.

40. Endo-Munoz L, Evdokiou A, Saunders NA. The role of osteoclasts and tumour-associated macrophages in osteosarcoma metastasis. Biochimica Et Biophysica Acta-Reviews on Cancer 2012; 1826: 434-442.

Reason for exclusion: This paper was a literature review and not a primary research looking at the outcome of interest to the review.

41. Killion JJ, Kleinerman ES, Wilson MR, Tanaka M, Fidler IJ. Sequential Therapy With Chemotherapeutic Drugs And Liposome-Encapsulated Muramyl Tripeptide - Determination Of Potential Interactions Between These Agents. Oncol Res 1992; 4: 413-418.

Reason for exclusion: This paper was a primary research paper, however, it did not meet the inclusion criteria as per the description mentioned in the published protocol.

42. Fidler IJ, Kleinerman ES. Therapy Of Cancer Metastasis By Systemic Activation Of Macrophages - From The Bench To The Clinic. Research in Immunology 1993; 144: 284287.

Reason for exclusion: This paper was not a primary research study and did not meet the inclusion criteria.

43. Kleinerman ES, Raymond AK, Bucana CD, et al. Unique Histological-Changes In Lung Metastases Of Osteosarcoma Patients Following Therapy With Liposomal Muramyl Tripeptide (CGP-19835A LIPID). Cancer Immunology Immunotherapy 1992; 34: 211-220.

Reason for exclusion: This primary research paper was not a quantitative study design but a descriptive/case series with no control group, which is not as per the description mentioned in the published protocol. 
44. Bielack SS, Carrle D, Hardes J, Schuck A, Paulussen M. Bone tumors in adolescents and young adults. Current Treatment Options in Oncology 2008; 9: 67-80.

Reason for exclusion: This paper was not a primary research study and did not meet the inclusion criteria.

45. Chou AJ, Gorlick R. Chemotherapy resistance in osteosarcoma: Current challenges and future directions. Expert Rev Anticancer Ther 2006; 6: 1075-1085.

Reason for exclusion: This paper was a literature review and not a primary research looking at the outcome of interest to the review.

46. Nardin A, Lefebvre ML, Labroquère K, Faure O, Abastado JP. Liposomal muramyl tripeptide phosphatidylethanolamine: Targeting and activating macrophages for adjuvant treatment of osteosarcoma. Curr Cancer Drug Targets 2006; 6: 123-133.

Reason for exclusion:This paper was not a primary research study and did not meet the inclusion criteria.

47. Federman N, Bernthal N, Eilber FC, Tap WD. The multidisciplinary management of osteosarcoma. Current Treatment Options in Oncology 2009; 10: 82-93.

Reason for exclusion: This paper was a literature review and not a primary research with the outcome of interest to the review.

48. Matthay KK. Osteosarcoma. Evidence-Based Pediatric Oncology: Third Edition: John Wiley and Sons, 2013; 14-24.

Reason for exclusion: This paper was not a primary research study and did not meet the inclusion criteria.

49. Ritter J, Bielack SS. Osteosarcoma. Annals of Oncology 2010; 21: vii320-vii325.

Reason for exclusion: This paper was not a primary research study and did not meet the inclusion criteria.

50. Bielack S, Carrle D, Casali PG. Osteosarcoma: ESMO clinical recommendations for diagnosis, treatment and follow-up. Annals of Oncology 2009; 20: iv137-iv139.

Reason for exclusion: This paper was a clinical practice guideline developed by the European Society for Medical Oncology (ESMO) and was not a primary research study of interest to review.

51. Chonn A, Cullis PR. Recent advances in liposomal drug-delivery systems. Current biology : CB 1995: 689-708.

Reason for exclusion: This paper was not a primary research study and did not meet the inclusion criteria.

52. Ferrari S, Palmerini E. Adjuvant and neoadjuvant combnation chemotherpay for osteogenic sarcoma. Curr Opin Oncol 2007; 19: 341-346. 
Reason for exclusion: This paper was a literature review and not a primary research with the outcome of interest to the review.

53. Anninga JK, Gelderblom H, Fiocco M, et al. Chemotherpeutic adjuvant treatment for osteosarcoma: Where do we stand? Eur J Cancer 2011; 47: 2431-2445.

Reason for exclusion: This paper was a literature review and not a primary research with the outcome of interest to the review.

54. Kleinerman ES, Snyder A, Jaffe N. Influence of Chemotherpay Administration on Monocytes Activation by Liposomal Muramyl Tripeptide Phosphatidylethanolamine in Children With Osteosarcoma. Journal of Clinical Oncology 1991; 9: 259-267.

Reason for exclusion: This paper was not a primary research study and did not meet the inclusion criteria.

55. Gano JB, Kleinerman ES. Liposomal MTP-PE. Journal of Pediatric Oncology Nursing 1994; 11: $161-163$.

Reason for exclusion: This paper was not a primary research looking at the outcome of interest to the review.

56. Gano J, Kleinerman E. Liposomal MTP-PE: A Promising New Biologic Response Modifier. Oncol Nurs Forum 1995; 22: 809-816.

Reason for exclusion: This paper was an overview summary of MTP-PE's history, mechanism of action, clinical trials and side effects that provide the knowledge to future nursing research and not a primary research looking at the outcome of interest to the review.

57. Mori K, Ando K, Heymann D. Liposomal muramyl tripeptide phosphatidyl ethanolamine: a safe and effective agent against osteosarcoma pulmonary metastases. Expert Rev Anticancer Ther 2008; 8: 151-159.

Reason for exclusion: This paper was a literature review focused on drug profile and not a primary research looking at the outcome of interest to the review.

58. Anderson P. Liposomal muramyl tripeptide phosphatidyl ethanolamine: ifosfamide-containing chemotherpay in osteosarcoma. Future Oncol 2006; 2: 333-343.

Reason for exclusion: This paper was a literature review on drug evaluation and not a primary research looking at the outcome of interest to the review.

59. Bramwell V. Metastatic ostoesarcoma: a review of current issues in systemic treatment. Sarcoma 1997; 1: 123-130.

Reason for exclusion: This paper was a literature review and not a primary research looking at the outcome of interest to the review.

60. Ando K, Mori K, corradini N, Redini F, Heymann D. Mifamurtide for the treatment of nonmetastatic osteosarcoma Expert Opin Pharmacother 2011; 12: 285-292. 
Reason for exclusion: This paper was a literature review focused on drug evaluation and most recent findings about mifamurtide treatment and its therapeutiv application. Also, it was not a primary research looking at the outcome of interest to the review.

61. Anderson PM, Meyers P, Kleinerman E, et al. Mifamurtide in Metastatic and recurrent osteosarcoma: A patient access study with pharmacokinetics, pharmacodynamic, and safety assessments. Pediatr Blood Cancer 2014; 61: 238-244.

Reason for exclusion: This paper was a primary research study but, did not meet the inclusion criteria.

62. Gill J, Ahluwalia MK, Geller D, Gorlick R. New targets and approaches in osteosarcoma. Pharmacology \& therapeutics 2013; 137: 89-99.

Reason for exclusion: This paper was a literature review and not a primary research looking at the outcome of interest to the review.

63. Bielack SS. Osteosarcoma: Time to move on? Eur J Cancer 2010; 46: 1942-1945.

Reason for exclusion: This paper was an editorial letter which addressed some comments on general questions about the acceptancy of a new treatment as part of a standard therapy. Also, it was not a primary research looking at the outcome of interest to the review.

64. Redondo A, Cruz J, Lopez-Pousa A. SEOM clinical guidlines for the treatment of osteosarcoma in adults-2013. Clin Transi Oncol 2013; 15: 1037-1043.

Reason for exclusion: This paper was not a primary research study and did not meet the inclusion criteria.

65. Majo J, Cubedo R, Pardo A. Treatment of Osteosarcoma. A Review. Rev esp cir orthop traumatol 2010; 54: 329-336.

Reason for exclusion: This paper was a literature review and not a primary research looking at the outcome of interest to the review.

66. Wan J, Zhang X, Liu T, Zhang X. Strategies and developments of immunotherapies in osteosarcoma (Review). Oncology Letters 2016; 11: 511-520.

Reason for exclusion: This paper was a literature review and not a primary research looking at the outcome of interest to the review.

67. Brosa M, Garcia del Muro X, Mora J, et al. Economic Considerations On The Use Of Mifamurtide In The Treatment Of Osteosarcoma In Spain. Value in Health 2014; 17: A526A527.

Reason for exclusion: This paper was a literature review and not a primary research looking at the outcome of interest to the review. 
68. Winstone J, Chadda S, Ralston S, Sajosi P. Review and comparison of clinical evidence submitted to support European Medicines Agency market authorization of orphandesignated oncological treatments. Orphanet Journal of Rare Diseases 2015; 10.

Reason for exclusion: This paper was a literature review and not a primary research looking at the outcome of interest to the review.

69. Lamplot JD, Denduluri S, Qin J, et al. The Current and Future Therapies for Human Osteosarcoma. Current Cancer Therapy Reviews; 9: 55-77.

Reason for exclusion: This paper was a literature review and not a primary research looking at the outcome of interest to the review.

70. Porter JB, Desmond M, O'Donnell M, et al. Challenges of a post-authorisation safety study (PASS) in an orphan oncology indication. Pharmacoepidemiology and Drug Safety 2014; 23: 472.

Reason for exclusion: This paper was a literature review and not a primary research looking at the outcome of interest to the review.

71. Kebudi R, Ayan I, Ozger H, et al. Efficacy in six courses of nonmethotrexate three-drug chemotherapy and surgery in osteosarcoma: 25-year experience (Supplement). J of Clin Oncol 2015; 33(15):10050.

Reason for exclusion: This paper appeared to meet the inclusion criteria but was published as a supplement of published meeting abstracts and author details were unavailable. 


\section{Appendix VII: Characteristics of included studies}

\begin{tabular}{|c|c|c|c|c|c|c|c|}
\hline & Study & Setting & Sample demographics & Intervention/comparator & Outcomes & Main findings & Interpretation \\
\hline 1 & $\begin{array}{l}\text { Chou et al. }{ }^{1} \\
2009 \\
\text { Prospective RCT- } 2 \times 2 \\
\text { factorial design }\end{array}$ & $\begin{array}{l}\text { Outpatient from Intergroup study } \\
0133 \text { at Children's Cancer } \\
\text { Group (CCG) institutions/multi- } \\
\text { centred facility in the USA }\end{array}$ & $\begin{array}{l}\text { N=91 patients } \\
\text { Age: median age } 13 \text { yrs (range: } 1 \text { - } \\
30 \text { yrs) } \\
\text { Male=56 } \\
\text { Female=35 } \\
\text { Population: histological } \\
\text { confirmation not required, clinically } \\
\text { detectable high-grade metastatic } \\
\text { osteosarcoma }\end{array}$ & $\begin{array}{l}\text { Intervention Group } \\
\text { *Regimen A+: N=22 } \\
{ }^{*} \text { Regimen B+: N=24 } \\
\text { Comparison Group } \\
\text { Regimen A-: N=21 } \\
\text { Regimen B-: N=24 }\end{array}$ & $\begin{array}{l}\text { Mifamurtide-related } \\
\text { adverse events - } \\
\text { reported as Toxicity }\end{array}$ & $\begin{array}{l}\text { Based on the CCG toxicity and } \\
\text { Complications Criteria } \\
\text {-Grade } 3 \text { and 4: } \\
\text { Hematologic (WBC, ANC, Platelet, } \\
\text { HGB); Hepatic(AST, ALT, AP, total } \\
\text { bill); renal (creatinine, CrCl); GI } \\
\text { (stomatitis, nausea \& vomiting); } \\
\text { Cardiac rhythm; Nervous } \\
\text { (peripheral sensory, } \\
\text { centrocerebellar; skin; Hearing, } \\
\text { objection; infection; fever; } \\
\text { performance status } \\
53 \% \text { at 5-year } \\
42 \% \text { at 5-year }\end{array}$ & $\begin{array}{l}\text { Addition of mifamurtide to } \\
\text { chemotherapy regimen did not achieve } \\
\text { a statistically significant difference for } \\
E F S, \text { with } p=0.23 \text { and overall survival, } \\
\text { with } p=0.27 \text { in the metastatic cohort. } \\
\text { Certainly it reports a survival advantage } \\
\text { for patients that received mifamurtide, } \\
\text { with no evidence of interaction between } \\
\text { chemotherapy and mifamurtide. }\end{array}$ \\
\hline 2 & $\begin{array}{l}\text { Meyers et al. } \\
2008 \\
\text { Prospective RCT- } 2 \times 2 \\
\text { factorial design }\end{array}$ & $\begin{array}{l}\text { Outpatient university hospital } \\
\text { setting oncology department } \\
\text { and Children's Oncology Group } \\
\text { (COG) institutions [previously } \\
\text { known separately as Children's } \\
\text { Cancer Group (CCG) and the } \\
\text { Pediatric Oncology Group } \\
\text { (POG)] facility in the USA. }\end{array}$ & $\begin{array}{l}\mathrm{N}=662 \text { patients } \\
\text { Age: median age } 13 \text { yrs (range: } 1 \text { - } \\
30 \text { yrs) } \\
\text { Male=361 } \\
\text { Female=301 } \\
\text { Population: histologically } \\
\text { confirmed, high-grade non- } \\
\text { metastatic resectable } \\
\text { osteosarcoma }\end{array}$ & $\begin{array}{l}\text { Intervention Group } \\
\text { *Regimen A+: N=163 } \\
\text { *Regimen B+: N=168 } \\
\text { Comparison Group } \\
\text { Regimen A-: N=168 } \\
\text { Regimen B-: N=163 }\end{array}$ & $\begin{array}{l}\text { Overall survival } \\
\text { EFS } \\
\text { Recurrence of } \\
\text { osteosarcoma }\end{array}$ & $\begin{array}{l}81 \% \text { at } 4 \text {-year } \\
74 \% \text { at } 6 \text {-year } \\
66 \% \text { at } 4 \text {-year } \\
64 \% \text { at } 6 \text {-year } \\
217(33 \%) \\
\text { measured by chest X-ray } \\
\text { undertaken monthly, lung CT scan } \\
\text { repeated every } 3 \text { months, and/or } \\
\text { bone scans every } 6 \text { months }\end{array}$ & $\begin{array}{l}\text { The addition of mifamurtide to the } \\
\text { chemotherapy regimen resulted in a } \\
\text { statistically significant improvement in } \\
\text { overall survival, with } p=0.03 \text { and no } \\
\text { statistically significant improvement but } \\
\text { a trend toward better } E F S \text {, with } p=0.08 \text {. } \\
\text { Author concluded that this study will } \\
\text { require thoughtful consideration for } \\
\text { inclusion of mifamurtide in the treatment } \\
\text { of patients with osteosarcoma in the } \\
\text { future. }\end{array}$ \\
\hline 3 & $\begin{array}{l}\text { Kleinerman et al. }{ }^{3} \\
1995 \\
\text { Quasi-experimental study }\end{array}$ & $\begin{array}{l}\text { Phase II trial, Outpatient } \\
\text { rehabilitation setting at the } \\
\text { University of Texas in the USA }\end{array}$ & $\begin{array}{l}\mathrm{N}=49 \text { patients } \\
\text { Age: } 8-70 \text { yrs } \\
\text { Male }=23 \\
\text { Female=26 } \\
\text { Population: histologically proven } \\
\text { pulmonary metastatic and/or } \\
\text { recurrent osteosarcoma }\end{array}$ & $\begin{array}{l}\text { Intervention Group } \\
\text { Group 1': N=12 } \\
\text { Group 2: } \mathrm{N}=16 \\
\text { Comparison Historical Group } \\
\text { Group } 3^{3} \text { (re-enrolled): } \mathrm{N}=21\end{array}$ & $\begin{array}{l}\text { Mifamurtide-related } \\
\text { adverse events - } \\
\text { referred as clinical toxic } \\
\text { effects }\end{array}$ & $\begin{array}{l}\text { For single dose: } \\
\text { Chill: began } 15 \text { mins to } 1.5 \text { hrs } \\
\text { postinfusion and lasted upto } 1 \\
\text { hour. } \\
\text { Fever: } 1 \text { to } 3 \text { hrs postinfusion and } \\
\text { occurred within } 2 \text { hour of chills. } \\
\text { Headache: } 1 \text { to } 2 \text { hrs postinfusion. } \\
\text { For subsequent doses: } \\
\text { Grade } 1 \text { (delayed fatigue) }\end{array}$ & $\begin{array}{l}\text { Group } 2 \text { had a significant prolongation } \\
\text { in time to recurrence for PFS, with } \\
p<0.03 \text { compared to group } 3 \text {. However, } \\
\text { for survival after relapse, both the Group } \\
1 \text { and Group } 2 \text { had a significant median } \\
\text { survival time, with } p<0.01 \text { and } p<0.04 \\
\text { respectively. Author concluded that } \\
\text { mifamurtide deserves further } \\
\text { investigation in an adjuvant setting as }\end{array}$ \\
\hline
\end{tabular}




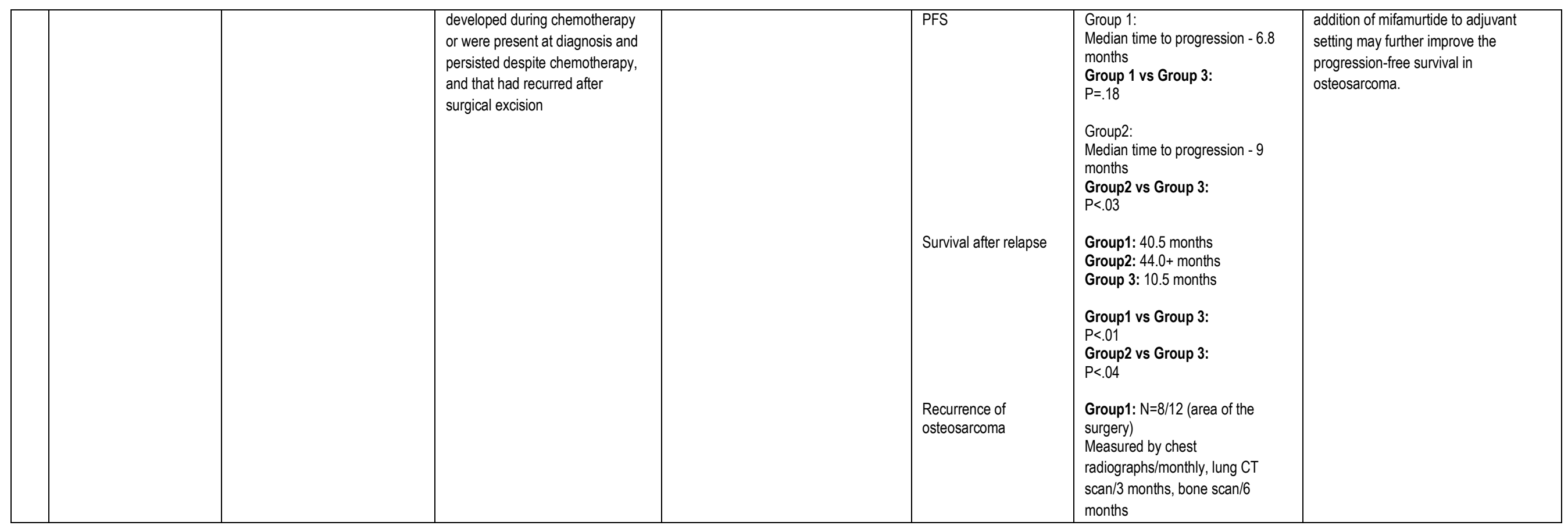

EFS: Event-free survival

PFS: Progression-free survival

Regimen A-: Patients treated with cisplatin, doxorubicin and HDMTX (high dose methotrexate)

Regimen A+: Patients treated with cisplatin, doxorubicin, HDMTX and mifamurtide

Regimen B-: Patients treated with cisplatin, doxorubicin, HDMTX and ifosfamide

Regimen B+: Patients treated with cisplatin, doxorubicin, HDMTX, ifosfamide and mifamurtide

${ }^{1}$ All patients in this group received mifamurtide $2 \mathrm{mg} / \mathrm{m}^{2}$ twice weekly for 12 weeks along with adjuvant chemotherapy

${ }^{2}$ All patients in this group received mifamurtide $2 \mathrm{mg} / \mathrm{m}^{2}$ twice weekly for 12 weeks and then once weekly for 12 weeks along with adjuvant chemotherapy

${ }^{3}$ All patients in this historical control group had received adjuvant chemotherapy alone consisting of one or more of the following agents: high-dose methotrexate with leucovorin, cisplatin, etoposide, ifosfamide, cyclophosphamide, and

vincristine

* Mifamurtide was administered intravenously $2 \mathrm{mg} / \mathrm{m}^{2}$ twice weekly for 12 weeks then once weekly for 24 weeks 